\title{
La montée du niveau de la mer d'ici 2100
}

\section{Scénarios et conséquences}

D. Lacroix, O. Mora, N. de Menthière, A. Béthinger

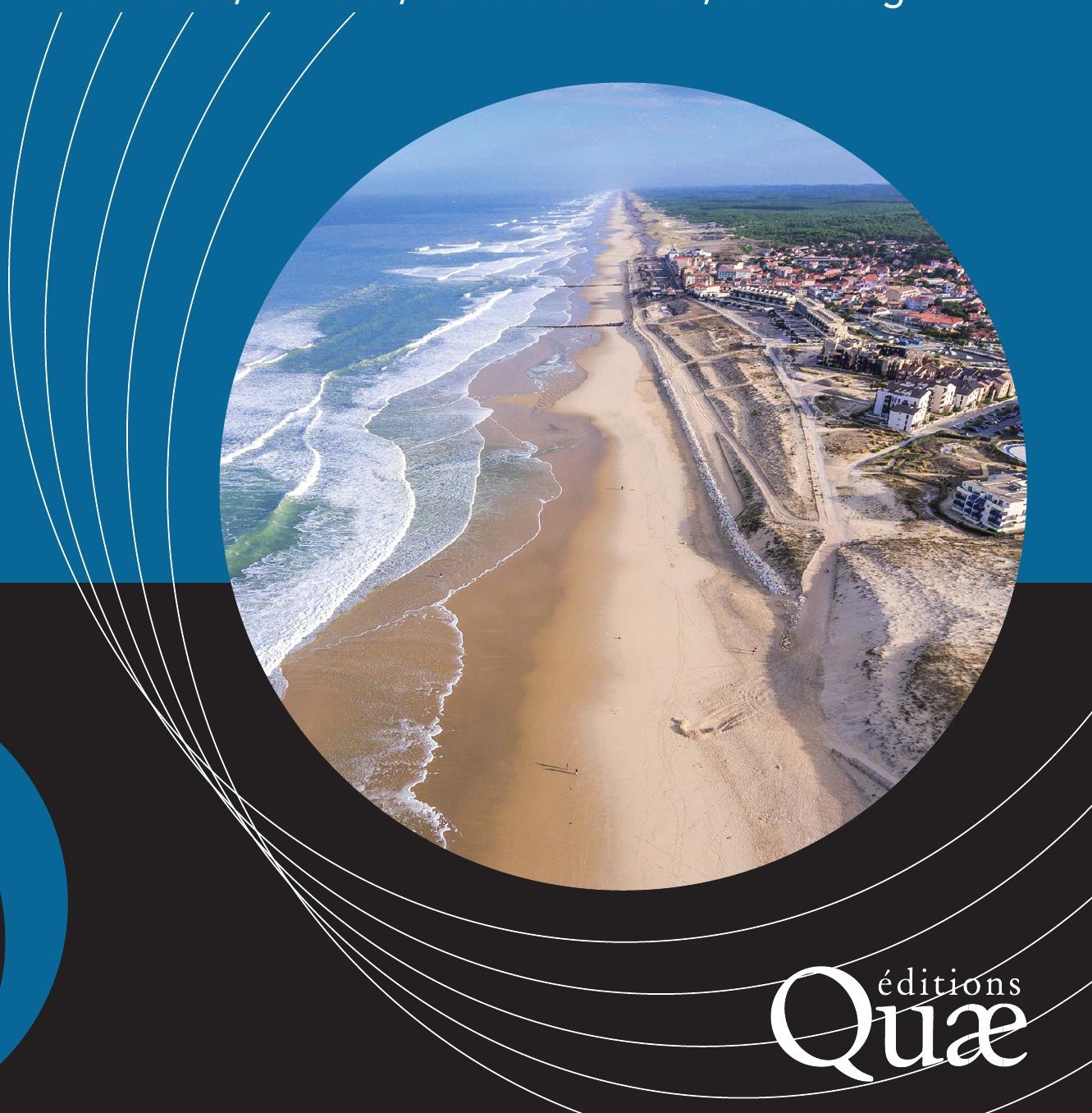





\section{La montée du niveau de la mer d'ici 2100 \\ Scénarios et conséquences}

Denis Lacroix, Olivier Mora, Nicolas de Menthière, Audrey Béthinger

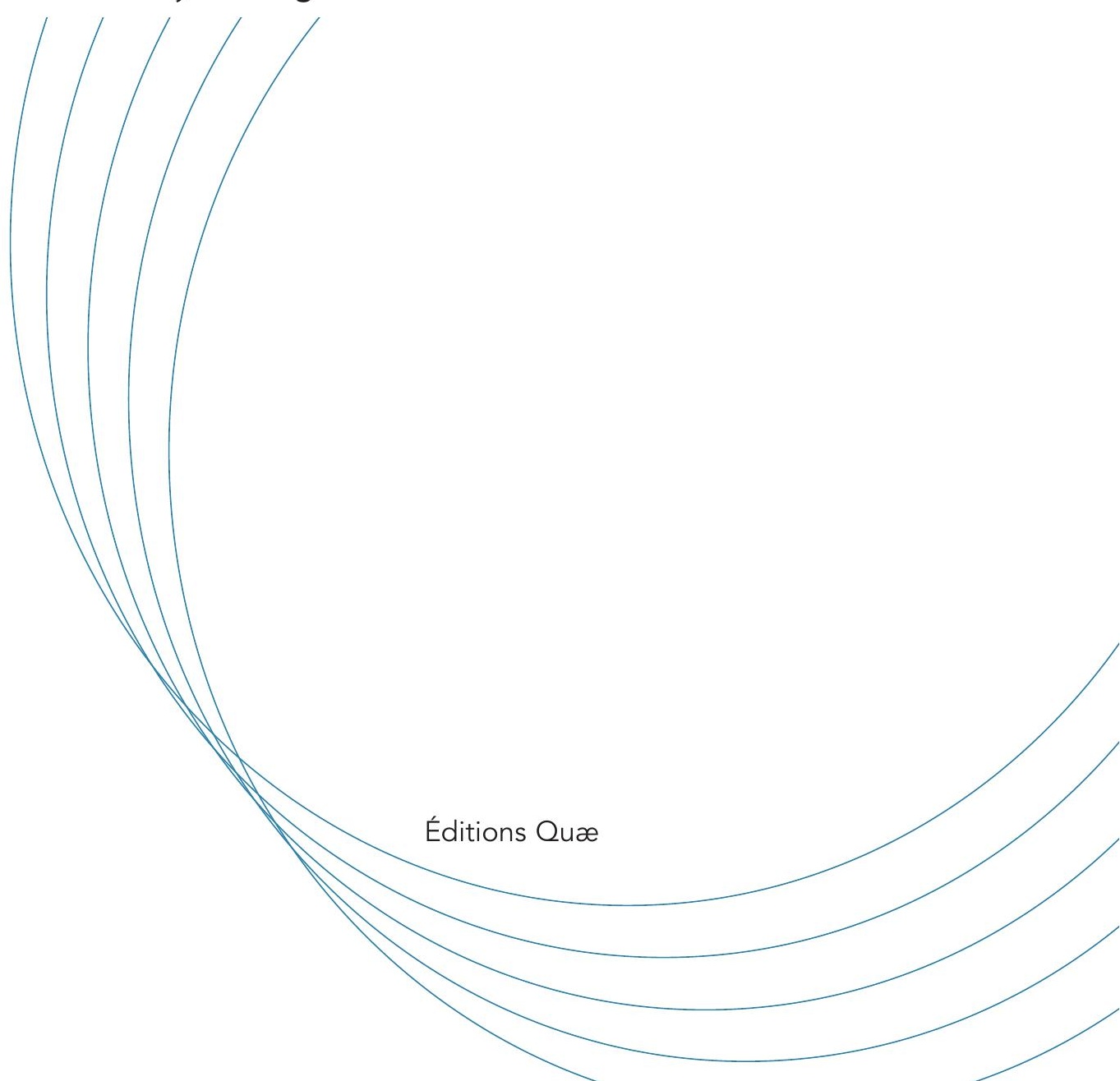




\title{
Collection Matière à débattre et décider
}

Filière forêt-bois et atténuation du changement climatique.

Entre séquestration du carbone en forêt et développement de la bioéconomie

A. Roux, A. Colin, J.-F. Dhôte, B. Schmitt, coord.

$$
\text { 2020, } 170 \text { p. }
$$

Quelle politique agricole commune demain ?

C. Détang-Dessendre, H. Guyomard, coord.

$$
\text { 2020, } 306 \text { p. }
$$

Quelles agricultures irriguées demain ?

S. Bouarfa, F. Brelle, C. Coulon, coord.

$$
\text { 2020, } 212 \text { p. }
$$

Les sols urbains sont-ils cultivables?

C. Mougin, F. Douay, M. Canavese, T. Lebeau, E. Rémy, coord.

2020, 228 p.

Stocker du carbone dans les sols français. Quel potentiel et à quel coût ?

S. Pellerin, L. Bamière, I. Savini, O. Réchaudière, coord.

$$
\text { 2021, } 232 \text { p. }
$$

En couverture : vue aérienne de la plage de Lacanau

(C) 2016 Observatoire de la Côte aquitaine/Com’by AVM

(C) Éditions Quæ, 2021

ISBN papier : 978-2-7592-3307-6

ISBN PDF : 978-2-7592-3308-3

ISBN ePub : 978-2-7592-3309-0

\author{
Éditions Quæ \\ RD 10 \\ 78026 Versailles Cedex, France \\ www.quae.com \\ www.quae-open.com
}

Le Code de la propriété intellectuelle interdit la photocopie à usage collectif sans autorisation des ayants droit. Le non-respect de cette disposition met en danger l'édition, notamment scientifique, et est sanctionné pénalement. Toute reproduction, même partielle, du présent ouvrage est interdite sans autorisation du Centre français d'exploitation du droit de copie (CFC), 20 rue des GrandsAugustins, Paris 6 . 


\section{Table des matières}

Préface 5

La prospective pour éclairer l'orientation des recherches environnementales 5

La montée du niveau de la mer : effet du dérèglement climatique,

défi sociétal majeur et objet scientifique interdisciplinaire 6

Introduction 9

La montée du niveau de la mer 11

Le développement des régions côtières 11

La problématique 12

Les enjeux d'une approche prospective 14

La méthode $\quad 15$

Partie I - Les scénarios d’évolution 17

1. Les contextes physiques d'élévation du niveau de la mer 18

2. Les trois familles de scénarios 22

Le processus d'élaboration 22

3. Schéma de positionnement des scénarios 35

Famille du "Déni » 36

Famille "Fragmentation persistante " $\quad 37$

Famille «Adaptation littorale » 38

Partie II - Focus territoriaux 41

4. La région Nouvelle-Aquitaine 45

Le littoral en Nouvelle-Aquitaine : diversifié, dynamique et attractif 45

Trois scénarios plausibles $\quad 49$

5. Les Pays-Bas 53

Un pays de terres basses et d'eau 53

Les scénarios possibles : quelles trajectoires d'adaptation

pour le « Dutch Delta» à 2100?

6. Le Vietnam 61

L'importance des deltas au Vietnam 61

Synthèse d'études récentes 63

Conclusion 66 
Partie III - Conséquences potentielles des scénarios

7. Pour l'environnement et les ressources naturelles 76

Les écosystèmes littoraux $\quad 77$

8. Pour l'agriculture et l'usage des terres dans les zones littorales 81 Les tendances actuelles dans les zones littorales 81

L'évolution de la disponibilité des terres agricoles littorales 82

Conséquences potentielles sur la place de la pêche et de l'aquaculture $\quad 84$

Conséquences potentielles sur la sécurité alimentaire 85

9. Pour les populations et les villes littorales 88

Une littoralisation des populations à l'échelle mondiale 88

Conséquences potentielles sur les zones urbaines littorales 91

Les stratégies d'adaptation et de protection des zones urbaines littorales 94

10. Pour la vie économique 96

Un enjeu complexe et multiforme 96

La difficile répartition des responsabilités 98

$\begin{array}{ll}\text { Conclusion } & 101\end{array}$

11. Pour la géopolitique 102

La géopolitique est très tôt liée à la mer 102

Vulnérabilité des populations et migrations 105

Enseignements à l'échelle de la France comme du monde 108

Conclusion 109

Des tendances lourdes et des scénarios inquiétants 109

Des marges de manœuvre 110

S'adapter, action nécessaire mais pas suffisante 111

Des raisons d'espérer 113

Remerciements 115

Annexe :

Méthodologie 116

Équipe projet et organisation des travaux 116

Les étapes de la construction du système 118

Structuration des visions d'avenir et schéma de positionnement des scénarios sur un plan $\quad 119$

Les composantes du système $\quad 119$

Le tableau morphologique $\quad 121$

Construction des scénarios $\quad 121$

Glossaire 122

Bibliographie 123 


\section{Préface}

\section{La prospective pour éclairer l'orientation des recherches environnementales}

CRÉÉE EN 2010, AllEnvi, l'alliance nationale de recherche pour l'environnement, rassemble les différents organismes et établissements français qui mènent des recherches environnementales. L'une de ses missions est d'orienter la programmation de la recherche sur les grands défis sociétaux relatifs à l'alimentation, à la biodiversité, au climat, à l'eau, à l'océan ou encore aux territoires ruraux ou urbains, et qui sont au cœur de la transition écologique.

La caractérisation de ces défis et des questions scientifiques qui leur sont liées repose naturellement sur une analyse de l'état de l'environnement et de ses multiples interactions avec la société : les pressions exercées par les activités humaines et leurs impacts, les services rendus par les écosystèmes, les perceptions et comportements individuels et collectifs... Cette analyse se décline à différentes échelles, du local au global ; elle considère la situation actuelle de l'environnement aussi bien que sa dynamique, son histoire et sa trajectoire future. La programmation de la recherche intègre ainsi, plus ou moins implicitement, une vision du futur qui lie les verrous méthodologiques ou technologiques à lever et les connaissances attendues aux risques à éviter, aux impacts à limiter ou aux bénéfices prévus...

Cette vision du futur s'appuie le plus souvent sur le prolongement de tendances déjà observées dans des registres différents : celui de la science, de ses concepts, méthodes ou infrastructures, comme celui des évolutions qui affectent la société et les systèmes naturels. Par exemple, la transition numérique, le dérèglement climatique, la croissance démographique, la fragmentation des paysages, l'intensification des activités humaines extractives... À l'horizon de quelques décennies, ces tendances sont cependant susceptibles de s'infléchir, de bifurquer ou de s'inverser : tout l'enjeu des transitions appelées par les objectifs du développement durable est d'ailleurs de modifier profondément certaines de ces tendances. En outre, les systèmes considérés sont si complexes qu'il serait vain de prétendre que ceux-ci vont se prolonger tels quels.

Il est donc aussi utile de développer d'autres méthodes pour explorer le futur et anticiper les conséquences de scénarios alternatifs, en vue d'identifier ou de prioriser les questions de recherche afin d'éviter des catastrophes ou de dessiner un avenir souhaitable avenir qu'il n'appartient pas aux chercheurs de choisir, mais d'éclairer. Cette démarche est, par exemple, celle du groupe d'experts intergouvernemental sur l'évolution du climat (GIEC), dont les différents scénarios dessinent des futurs climatiques contrastés, sur la base desquels des choix politiques sont réalisés. 
Avant même la création d'AllEnvi, plusieurs de ses membres s'étaient ainsi déjà dotés d'une capacité prospective dans leur domaine spécifique de compétences. C'est ainsi que le Cirad et l'Inra ont exploré le devenir des systèmes agricoles et alimentaires à l'horizon 2050 (AgriMonde, 2009, puis AgriMonde Terra, 2016) ${ }^{1}$. Autre exemple : en 2008, l'Ifremer s'est intéressé au futur des énergies marines renouvelables à l'horizon 2030². D'autres exercices portent plutôt sur « le mouvement interne » de la science et des technologies : cette démarche est solidement ancrée au CNRS, dans les différentes disciplines, ou encore au Cnes pour ce qui concerne l'espace.

Au sein d'AllEnvi, il est apparu utile de partager ces pratiques de la prospective et de créer un groupe de travail transversal dédié. Le premier projet de ce groupe, ScénEnvi, a porté sur l'analyse synthétique des grandes prospectives internationales sur l'environnement, aux échelles du monde ou des grands continents et aux horizons 2030, 2050 ou 2100 : l'examen des 99 études repérées et jugées pertinentes a permis d’identifier plus de 300 scénarios qui ont été regroupés en 11 grandes familles. Sans surprise, le dérèglement climatique est souvent présent dans ces scénarios, soit comme une conséquence de priorités d'une autre nature, par exemple économique, soit comme l'un des moteurs mêmes de l'évolution du monde. En revanche, l'océan - tout comme la forêt, d'ailleurs s'avère être l'un des grands absents des scénarios ainsi repérés.

\section{La montée du niveau de la mer : effet du dérèglement climatique, défi sociétal majeur et objet scientifique interdisciplinaire}

SANS QU'IL N'Y AIT DE RELATION causale explicite avec le constat précédent, le conseil des membres d'AllEnvi a décidé en 2017 que le deuxième projet collectif du groupe transversal « Prospective » porterait sur la montée du niveau de la mer : le présent ouvrage est le fruit de ce travail collectif.

Comme l'ont montré le rapport spécial du GIEC sur l'océan et la cryosphère ${ }^{4}$, le deuxième rapport des Nations Unies sur l'état mondial de l'océan ${ }^{5}$, ou encore le récent rapport du Conseil économique, social et environnemental sur la pêche durable en mer face au

1. https://www6.paris.inrae.fr/depe/Presentation/La-prospective

2. https://wwz.ifremer.fr/cop/content/download/15287/file/Ifremer_synthese-etude-prospective-EnRM. pdf

3. De Menthière N., Lacroix D., Schmitt B., Béthinger A. (coordinateurs). 2016. Visions du futur et environnement. Les grandes familles de scenarios analyse de prospectives internationales relatives à l'environnement. https://www.allenvi.fr/allenvi/actualites/actualites-2013-2018/2017/scenenvi-futurs-pour-la-planete 4. IPCC. 2019. The Ocean and Cryosphere in a Changing Climate. A Special Report of the Intergovernmental Panel on Climate Change. https://www.ipcc.ch/site/assets/uploads/sites/3/2019/12/SROCC_FullReport_ FINAL.pdf

5. United Nations. 2021. The Second World Ocean Assessment. 2 volumes : Vol. $1:$ https://www.un.org/ regularprocess/sites/www.un.org.regularprocess/files/2011859-e-woa-ii-vol-i.pdf Vol. 2 : https://www. un.org/regularprocess/sites/www.un.org.regularprocess/files/2011859-e-woa-ii-vol-ii.pdf 
changement climatique ${ }^{6}$, les interactions entre l'océan et le climat sont nombreuses. La montée du niveau de la mer n'est donc qu'un aspect des effets du dérèglement climatique sur l'océan et sur les activités humaines qui lui sont plus ou moins directement liées. Ce phénomène et ses conséquences sont cependant particulièrement intéressants à étudier dans une démarche prospective :

- D'abord parce que l'élévation moyenne du niveau de la mer est inexorable à l'échelle de ce siècle et que les projections issues de multiples travaux scientifiques internationaux la réévaluent régulièrement et montrent qu'elle a même tendance à s'accélérer : on sait maintenant qu'il est ainsi possible qu'elle dépasse un mètre avant 2100, et ce même si la hausse de la température moyenne parvenait à être limitée à 1,5 ou 2 degrés par des politiques vigoureuses de réduction des émissions de gaz à effet de serre ;

- ensuite, parce que les zones littorales directement affectées par cette élévation du niveau de la mer sont de plus en plus peuplées, si bien que ses effets physiques et écologiques directs (recul du trait de côte, salinisation des nappes phréatiques, submersions temporaires ou permanentes de terres basses, pollutions liées à ces submersions...) auront des conséquences considérables pour les populations et les sociétés concernées (dommages aux infrastructures et aux villes côtières, accroissement des risques et des coûts de protection, d'assurance et de réparation, déplacements de populations...) : les projections, selon diverses hypothèses, des scénarios et de leurs impacts conduisent ainsi à une majorité de situations graves ou extrêmes à l'horizon 2100 ;

- enfin, parce que la conjonction de l'inéluctabilité du phénomène, de son irréversibilité - du moins à l'échelle de quelques générations humaines - et de l'ampleur de ses conséquences invite à anticiper les menaces, à s'y préparer, à renforcer les politiques d'atténuation du dérèglement climatique et à initier des politiques d'adaptation dont l'enjeu est de réduire les risques de catastrophes et les coûts humains et économiques afférents. Ce besoin est d'autant plus grand que la montée du niveau de la mer et ses effets sont variables selon les régions du monde et qu'ils appellent donc des réponses différenciées.

Le travail mené par le groupe Prospective d’AllEnvi illustre ainsi la diversité des scénarios possibles, selon l'efficacité des politiques d'atténuation globale du dérèglement climatique, d'après la nature des territoires littoraux concernés et en fonction de la vigueur et de la précocité des politiques d'adaptation. Il ne prétend pas prédire l'avenir mais éclairer les choix : il invite tous les acteurs concernés à la lucidité, à l'anticipation et à la mobilisation collective.

Pour ce qui concerne l'orientation des recherches, l'ouvrage conclut naturellement à l'intérêt d'approches interdisciplinaires, qui soient à la mesure de la complexité du phénomène. Il propose ainsi quelques grands domaines prioritaires qui s'inscrivent dans le développement de la « science de la durabilité » qu'AllEnvi et ses membres appellent de leurs vœux :

6. CESE. 2021. Quelle pêche durable en mer face au changement climatique ? https://www.lecese.fr/ travaux-publies/quelle-peche-durable-en-mer-face-au-changement-climatique 
- La poursuite de l'étude du phénomène «naturel » considéré dans toute sa complexité : ses déterminants, sa vitesse, son ampleur et ses variations spatiales, ses impacts physiques et écologiques, ou encore ses interactions avec d'autres phénomènes naturels (la subsidence de certains littoraux, les liens avec l'hydrologie continentale);

- les perceptions et représentations individuelles et collectives de la montée du niveau de la mer. En appréhender la diversité, par exemple culturelle, est essentiel pour la construction de « solutions » qui conviennent aux populations concernées ;

- la gouvernance des territoires, considérée à différentes échelles spatiales et à différents horizons temporels, et selon leur nature rurale ou urbaine. De nombreuses disciplines des sciences sociales et humaines sont ici nécessaires pour comparer les coûts respectifs de l'action et de l'inaction ;

- l'étude des options et leviers envisageables dans le cadre de politiques d'adaptation, dont la conception, le suivi et l'évaluation supposent de mobiliser les sciences de l'ingénieur, les sciences humaines et sociales et les sciences de la nature.

François Houllier

P-DG de l'Ifremer

Président d'AllEnvi 


\section{Introduction}

LE CHANGEMENT CLIMATIQUE est maintenant une réalité reconnue par la majorité des décideurs et de l'opinion publique. Si certaines de ses conséquences, comme les vagues de chaleur ou la fonte des glaciers, apparaissent clairement associées à ce changement, un autre de ses effets, la montée du niveau de la mer, n'est pas toujours considérée comme liée à ce changement. Pourtant, cette élévation apparaît aujourd'hui comme l'une des conséquences majeures du changement climatique, même si son ampleur et ses impacts à terme restent encore difficiles à quantifier et à positionner dans le temps. Il apparaît donc utile de tenter de clarifier les données de cet enjeu car, si les impacts de cette élévation sont encore faibles aujourd'hui, la majorité des projections portent à croire que la hausse du niveau de la mer va s'accélérer au cours des décennies, voire des siècles à venir. Même si l'on reste dans des projections moyennes de cette hausse à l'horizon du siècle, les conséquences risquent d'être considérables en zone côtière, voire catastrophiques dans certaines régions, dans de nombreux domaines comme le trait de côte lui-même, les écosystèmes littoraux, l'habitat humain, les infrastructures industrielles et portuaires, l'agriculture, le tourisme... De fait, l'interface terre-mer, pris au sens large, est un espace d'autant plus fragile qu'il est dépendant du niveau de la mer. Or le développement des activités humaines dans cet espace est facilité par l'accessibilité à la mer et par la disponibilité de zones planes constructibles. Les premiers ports étaient sur des côtes rocheuses offrant des rades naturelles faciles à défendre avec des fortifications élevées (Marseille, Gênes, Constantinople, Alger...). Mais la maritimisation de l'économie à partir du xvı ${ }^{e}$ siècle a conduit à développer les ports à grande échelle et à localiser les activités associées dans des zones côtières basses. Aujourd'hui, cet avantage est devenu une vulnérabilité. De nombreux grands ports dans le monde, et les grandes villes qui se sont développées autour, ont déjà entrepris des travaux de protection contre la submersion et les inondations (Londres, Rotterdam, Bangkok...) et certains pourraient même être relocalisés dans leur ensemble, comme Djakarta. Par ailleurs, le thalassotropisme de l'habitat, notamment celui lié au tourisme, a pris des proportions considérables après 1945 , avec une préférence pour un accès facile à la mer, créant ainsi une nouvelle source de vulnérabilité. L'attractivité de la « vue sur la mer » a éclipsé toute considération rationnelle de vue sur l'avenir.

Le croisement de ces deux dynamiques, celle de la hausse inexorable du niveau de la mer et celle de la croissance continue des activités humaines dans les zones basses côtières constitue donc un enjeu majeur avant même la fin de ce siècle et encore plus au-delà (Bonneil et Fressoz, 2013).

La montée des eaux ignore les frontières et, si elle peut être différente selon les mers ou les régions océaniques, elle sera indépendante des efforts locaux pour la ralentir, voire pour tenter de la juguler, si ceux-ci ne restent le fait que de quelques États. C'est donc 
une question de portée mondiale qui demande des actions internationales concertées ou au moins convergentes pour aboutir à des résultats dès lors que l'on veut faire plus que se protéger localement contre les risques de submersion.

L'imprécision actuelle des projections justifie le recours à la prospective pour ouvrir dans ce domaine des perspectives de réflexion et d'action. Même si la tendance est incontestable, les projections sur la hausse du niveau de la mer à divers horizons temporels sont imprécises pour deux raisons. La première est que les mécanismes physiques, chimiques et biologiques mis en jeu ne sont pas totalement explicités et, par conséquent, leur modélisation et leurs projections dans le temps restent peu précises. La seconde raison est que la cause de cette hausse est le changement climatique, phénomène dont l'ampleur et la vitesse sont directement liées aux activités anthropiques et aux choix politiques, industriels et comportementaux de milliards d'individus. Sur la durée d'une décennie, $a$ fortiori sur un siècle, les évolutions des choix de gouvernance climatique, industrielle, technologique, économique et sociétale, à l'échelle régionale ou mondiale, restent largement conjecturales. Dans cette configuration, l'approche prospective offre l'intérêt de proposer des scénarios d'évolution possible ou probable des systèmes côtiers, via des méthodes rigoureuses d'analyse et de synthèse, avec des hypothèses explicites et ouvertes à la discussion. Ces méthodes permettent de tracer un champ des chaînes de causalités possibles, et d'en tester la robustesse en modifiant le jeu des variables motrices et de la combinatoire de leurs hypothèses. Elles donnent aussi des matériaux de discussion pour une meilleure prise de conscience de cette évolution à l'échelle mondiale et pour les choix de priorités stratégiques en termes d'aménagement comme de programmes de recherche. C'est dans ce cadre que l'Alliance des sciences de l'environnement du ministère de la Recherche et de l'Innovation français (MESRI), via son conseil, a demandé en février 2017 à son groupe transversal de prospective (voir encadré), de réfléchir à cette problématique et de conduire une étude intitulée : "La montée du niveau de la mer : conséquences et anticipations ; l'éclairage de la prospective ».

L'Alliance nationale de recherche pour l'environnement (AllEnvi) fédère les forces de recherche publique pour programmer et coordonner la stratégie scientifique française dans le domaine de l'environnement. Réunissant vingt-huit partenaires, elle est gouvernée par un Conseil rassemblant autour des présidents ou directeurs généraux de ses douze membres fondateurs (a), deux représentants de ses seize membres associés.

La mission d'AllEnvi est d'éclairer la recherche dans sa mission d'acquérir les connaissances et de construire les méthodes nécessaires pour répondre aux questions qui se poseront dans le futur. De fait, de nombreux programmes de recherche à l'échelle d'un laboratoire, d'un département, d'un institut ou d'un ministère intègrent une certaine vision du futur de ceux qui les définissent. Cependant, la tendance naturelle, au sein d'une structure de recherche, est de ne raisonner qu'en tendances dites "lourdes", donc peu contestables, sur des situations projetées dites " médianes ", ce qui revient à éviter les scénarios pessimistes ou les 
ruptures. Or, pour montrer l'intérêt d'un programme de recherche, il peut être utile de le resituer dans plusieurs visions de futurs possibles ou de référence à l'horizon 2030 ou 2050, car il n'est pas rare qu'une technique arrive à maturité en trente ans et plus (par exemple, l'informatique) dans un environnement économique et technologique mondialisé. Pour éclairer ses choix et ceux de ses membres, l'Alliance s'est donc dotée d'un groupe transversal de prospective constitué de représentants mandatés par chacun des organismes partenaires.

(a) BRGM, CEA, Cirad, CNRS, CGE, Ifremer, Ifsttar, INRAE, IRD, Irstea, Météo France, MNHN

\section{La montée du niveau de la mer}

LA MESURE DE LA MONTÉE DU NIVEAU de la mer et l'étude des phénomènes qui sont impliqués dans son équilibre dynamique n'ont cessé de se perfectionner depuis un siècle, en complément des études sur la température. De nombreux capteurs ont été mis au point, des satellites aux flotteurs in situ, sur toutes les côtes du monde y compris en Antarctique. En parallèle, des programmes de traitement des données et de modélisation ont permis d'intégrer des masses croissantes d'informations et de faire des projections sur les décennies à venir avec des marges d'incertitude acceptables pour les décideurs. Cette mondialisation des efforts de compréhension de la machinerie climatique et océanique, avec une responsabilité particulière confiée au groupe international d'étude du climat (GIEC) créé en 1989, a permis d'actualiser régulièrement des projections possibles du niveau de la mer à divers horizons temporels. Ainsi, son dernier rapport donne une fourchette d'élévation du niveau moyen de la mer allant de 29 à $110 \mathrm{~cm}$ selon les hypothèses minimales ou maximales de réchauffement climatique (Oppenheimer et al., 2019). Mais la valeur haute des projections $(+110 \mathrm{~cm}$ ) pourrait être dépassée, en particulier si la fonte des calottes glaciaires devait continuer à s'accélérer (Labeyrie, 2015 ; Bamber et al., 2019). Ces projections ont longtemps été négligées par la plupart des pays pour des raisons d'inertie politique et d'opposition des intérêts économiques, mais elles sont peu à peu prises au sérieux au fil des conférences internationales sur le climat et des observations des dérèglements climatiques. Cet enjeu concerne directement l'Europe car nombre de plaines basses et grands deltas sont déjà affectés : Rhône, Pô, Rhin-Meuse, Tamise... (Chaumillon et al., 2014).

\section{Le développement des régions côtières}

DepuIS 1945, LeS ACtivités humaines se sont développées plus rapidement en zone côtière qu'ailleurs et dans tous les domaines : l'habitat, l'exploitation de ressources vivantes, minérales et énergétiques, les infrastructures lourdes pour l’industrie (usines, 
centrales d'énergie, raffineries...) et le transport (routes et ports), le commerce, les activités militaires, les loisirs... à de larges échelles et souvent avec une forte intensité. Ces espaces apparaissent aussi comme des opportunités de croissance dite «bleue » avec notamment la multiplication des parcs éoliens marins.

La première tendance lourde dans cet espace est l'accroissement continu de la population littorale. On estime que $20 \%$ de la population mondiale vit à moins de 20 km d'une côte, cette proportion atteignant 50 \% si l'on étend la zone côtière à une profondeur de 100 km (Wong et al., 2014). Les risques d'exposition à des épisodes de submersion ou d'inondation affectent déjà plus de 625 millions de personnes habitant à moins de $10 \mathrm{~m}$ au-dessus de la mer. Ce chiffre devrait approcher du milliard vers 2050 (Neumann et al., 2015). Les pays les plus vulnérables sont principalement situés en Asie, où cinq pays concentrent $50 \%$ des populations les plus exposées. Les ports, interfaces vitales du commerce maritime mondial sont également menacés : 12 des 20 plus grands ports du monde sont devenus vulnérables, en Asie mais aussi en Europe (Londres et Rotterdam par exemple).

Au cours des 70 dernières années, l'artificialisation des côtes à des fins de développement économique a conduit à densifier le tissu urbain, touristique et industriel à proximité de la mer et donc à multiplier les risques d'impacts des événements météorologiques extrêmes. Les exemples des catastrophes de la ville de La Nouvelle-Orléans (1 850 morts lors du passage de l'ouragan Katrina en 2005), La Faute-sur-mer (29 morts lors de la tempête en 2010) et de Fukushima (tsunami en 2011) illustrent bien cette vulnérabilité croissante.

Ce développement rapide des zones côtières se traduit par une artificialisation souvent mal contrôlée des zones littorales, qui impacte l'activité agricole, et entraîne aussi des pressions croissantes sur les ressources, qu'elles soient minérales (l'extraction des granulats, par exemple), énergétiques (pétrole et gaz) ou vivantes (pêche, biodiversité). Or, la mer côtière et les zones humides fournissent une grande diversité de services écosystémiques non marchands (nurseries d'espèces marines, puits de carbone, filtration ou absorption de composés toxiques, recyclage...), ce qui contribue à la durabilité des écosystèmes productifs et à la qualité générale de l'environnement. Ces services sont vitaux pour nombre d'activités comme le tourisme, l'aquaculture (source de $16 \%$ des protéines dans le monde, hors pêche), mais ils sont menacés et risquent de l'être encore plus à l'avenir. L'interface terre-mer, zone riche, active et peuplée, est donc « en première ligne » des changements climatiques globaux.

\section{Laproblématique}

CET ESPACE CôtIER au sens large, caractérisé par une forte concentration de population et une croissance urbaine et économique rapide concentre aussi les incertitudes et les risques. Associée à la réduction des apports en eaux douce, notamment dans de nombreux pays subtropicaux, la submersion des terres basses s'accompagne d'une salinisation 
croissante des sols et des nappes phréatiques (Hereher, 2010). Ainsi, ce phénomène, observé depuis 30 ans dans le bas delta du Nil, en Égypte, comme dans la plupart des deltas d'Asie, notamment celui du Mékong, a fait disparaître de vastes zones de production agricole. A contrario, il a permis à l'aquaculture en eau saumâtre de se développer à grande échelle avec une production passée sur la période de cent mille à un million et demi de tonnes par an.

Les effets de l'élévation annoncée du niveau de la mer ne se limiteront pas à la frange littorale. Ils se feront de plus en plus sentir à l'intérieur des terres ; par exemple, par une difficulté accrue d'écoulement des eaux, augmentant les surfaces inondées et par conséquent les dégâts lors des crues; ou une modification de l'hydromorphologie des fleuves, dont le profil en long sera recalé par des dépôts de sédiments modifiant les écosystèmes aquatiques et la sécurité de certains ouvrages hydrauliques. De même, l'apparition de vastes zones humides, voire de lagunes ou lacs saumâtres de plus en plus à l'intérieur des terres pourrait avoir des conséquences en termes de santé humaine, notamment par le développement des moustiques, vecteurs de maladies.

Si des risques environnementaux classiques, comme des feux de forêts, des tempêtes ou des pandémies, peuvent être à peu près maîtrisés via divers moyens technologiques et des mesures de gouvernance, le risque climatique et, avec lui, la montée de la mer présentent un caractère d'exception parce qu'ils sont globaux, irréversibles, et que leur maîtrise exigerait un « nouveau contrat social » à l’échelle mondiale (Beck, 1986).

Des centaines de millions de personnes ainsi que toute l'économie littorale associée sont déjà concernées par la montée du niveau marin. Cependant, les contrastes sont forts entre des pays riches, comme les Pays-Bas capables d'investissements d'anticipation, et les pays pauvres, comme le Bangladesh, n'ayant d'autre choix que celui de la migration avec toutes les difficultés et tensions que cela engendrera. II n'est cependant pas simple d'élaborer une stratégie cohérente de long terme face à ce phénomène, car toute la gamme des réactions humaines peut être observée dans les débats qui croisent des intérêts de court terme et des tendances de long terme : déni du problème, indifférence, acceptation fataliste, résistance au changement climatique, réaction pragmatique, volonté d'anticipation de long terme. Dans la pratique, le plus souvent, il ne s'agit pas de construire un mur mais de conserver une interface vivante avec la mer et de restructurer en profondeur les espaces côtiers. Que ce soit à l'échelle d'une commune, d'un État ou d'une organisation internationale, les décisions finales sont, le plus souvent, des compromis, avec une grande difficulté d'estimation du ratio coûts/bénéfices sur le long terme. De fait, même s’il existe déjà plusieurs conventions internationales traitant divers aspects de ce problème global (Ramsar 1971, Barcelone 1976, Ospar 1998...), les principes tendant à éviter les perturbations les plus graves, à réduire et à compenser leurs effets sont toujours difficiles à traduire en actions concrètes et efficaces (Aronson et al., 2007).

De fait, il n'est pas aisé de proportionner l'échelle des travaux aux menaces car de nombreux paramètres interviennent, à commencer par l'horizon temporel de fiabilité de la protection. Faut-il de gros travaux pour être à l'abri pour un siècle ou une simple digue 
pourrait-elle suffire pendant quelques décennies, le temps de s'adapter ? Les conséquences environnementales, sociales et économiques de la montée du niveau de la mer vont dépendre de l'ampleur de cette montée, des caractéristiques initiales et des usages actuels et futurs des espaces littoraux considérés, et surtout de la capacité de réaction et d'anticipation des instances de gouvernance. La combinaison de ces trois dimensions nécessite d'explorer plusieurs futurs possibles aux conséquences contrastées.

\section{Les enj eux d'une approche prospective}

COMMENT CONCEVOIR LE LITTORAL anthropisé sur le long terme face à la menace croissante de la montée du niveau de la mer, au moins jusqu'à 2100, en prenant en compte le risque de " tempête parfaite » (conjonction de toutes les menaces potentielles) sur au moins certains points du littoral ? Comment garder compatibles l'expansion des activités maritimes avec la sécurité et la pérennité des milieux ? Les échelles de perturbation de l'environnement liées à la montée de la mer sont si considérables qu'elles font apparaître de forts besoins en connaissances dans de nombreux domaines de recherche. Se projeter à 2100 est donc devenu nécessaire pour décider de manière pertinente à l'horizon 2030.

La prospective irrigue déjà la planification à moyen terme de diverses activités maritimes. Mais la gestion durable du littoral face à un changement aussi global que la montée du niveau de la mer ne pourra émerger sans démarche collaborative alliant sciences économiques et sociales, sciences biologiques, biotechniques et biophysiques, technologies et projection de choix de gouvernance dans de nombreux domaines : environnement, société, économie, urbanisation, industries, tourisme... De fait, l'adaptation au changement climatique global, y compris la dimension marine, constitue déjà parmi les plus importants thèmes de recherche à l'échelle européenne. Ce changement impose donc d'adapter les activités humaines sur les terres basses côtières à un horizon au moins de moyen terme. Cette restructuration, qui devra être co-construite avec les acteurs locaux, sera d'autant plus durable et sûre qu'elle sera anticipée, planifiée et accompagnée.

Outil assez récent au service de la gouvernance, avec l'intérêt d'une sorte de « modélisation » des choix stratégiques et d'estimation de leurs conséquences (Godet et Durance, 2008), l'analyse prospective contribue à faciliter les débats entre porteurs d'enjeux, à la sélection collective de mesures sans regret et, enfin, à l'appropriation par toutes les parties des actions nécessaires à mener à l'échelle régionale, quand ce n'est pas encore possible à l'échelle mondiale. 


\section{La méthode}

Le LARGE ÉVENTAIL DES VITESSES de montée du niveau de la mer et la variété des conséquences et des réactions possibles justifient le recours à la méthode des scénarios. Cette méthode est une des mieux adaptée pour traiter une problématique complexe, avec de larges incertitudes sur les phénomènes en jeu et sur le temps long du siècle. Dans cette étude, le nombre élevé des variables motrices (23) et la large gamme de variation des hypothèses pour chaque variable ont permis de générer des scénarios robustes, plausibles et contrastés.

Il est donc proposé de mener une prospective à horizon éloigné (2100), visant à élaborer des représentations contrastées des futurs possibles, en articulant l'échelle mondiale à laquelle se déroule le phénomène étudié, et l'échelle régionale avec des cas-types locaux. Ces illustrations, par une diversité de territoires impactés, permettent de montrer diverses configurations littorales dans leur contexte géopolitique, économique et social, et de mesurer les conséquences de différentes politiques. Les trois illustrations d'exposition d'un territoire à la montée de la mer concernent une première situation-type en Asie, au Vietnam, une deuxième dans le pays européen le plus menacé, les Pays-Bas et une troisième dans une région française vulnérable, la Nouvelle-Aquitaine.

Les résultats devraient aider tout décideur à prendre la mesure des enjeux à l'échelle mondiale comme dans son périmètre de responsabilité. Il s'agit d'abord d'éclairer les besoins en concertation, en acquisition complémentaire de connaissances et en financement. Ensuite, cette approche de prospective facilite la programmation des actions à mener et la mobilisation des partenariats nécessaires à toute échelle, à court, moyen et long terme.

Le groupe d'experts :

- Bernardot Marc [Université du Havre]

- Hérivaux Cécile (BRGM)

- Bertin Xavier

- Le Cozannet Gonéri (BRGM) (CNRS/GT Mer AllEnvi)

- Lobry Jérémy (IRSTEA)

- Carnus François (BRL Ingénierie)

- Meerpoel Matthieu (ICL)

- Cattaneo Antonio (lfremer/GT

- Meyssignac Benoît (LEGOS) Risques AllEnvi)

- Petitet Sylvain (Cerema)

- Devès Maud (AFPCN/IPGP)

- Rocle Nicolas (INRAE)

- Dörfliger Nathalie (BRGM)

- de Rouw Anneke (IRD)

- Durand Gaël (CNRS)

- Duval Anne-Marie (Cerema)

- Guedon Sylvine (IFSTTAR)

- Salas y Melia David (Météo France)

- Vagner Marie (CNRS)

- Valentin Christian (IRD/GT Sols AllEnvi) 



\section{Partie I}

Les scénarios

d'évolution

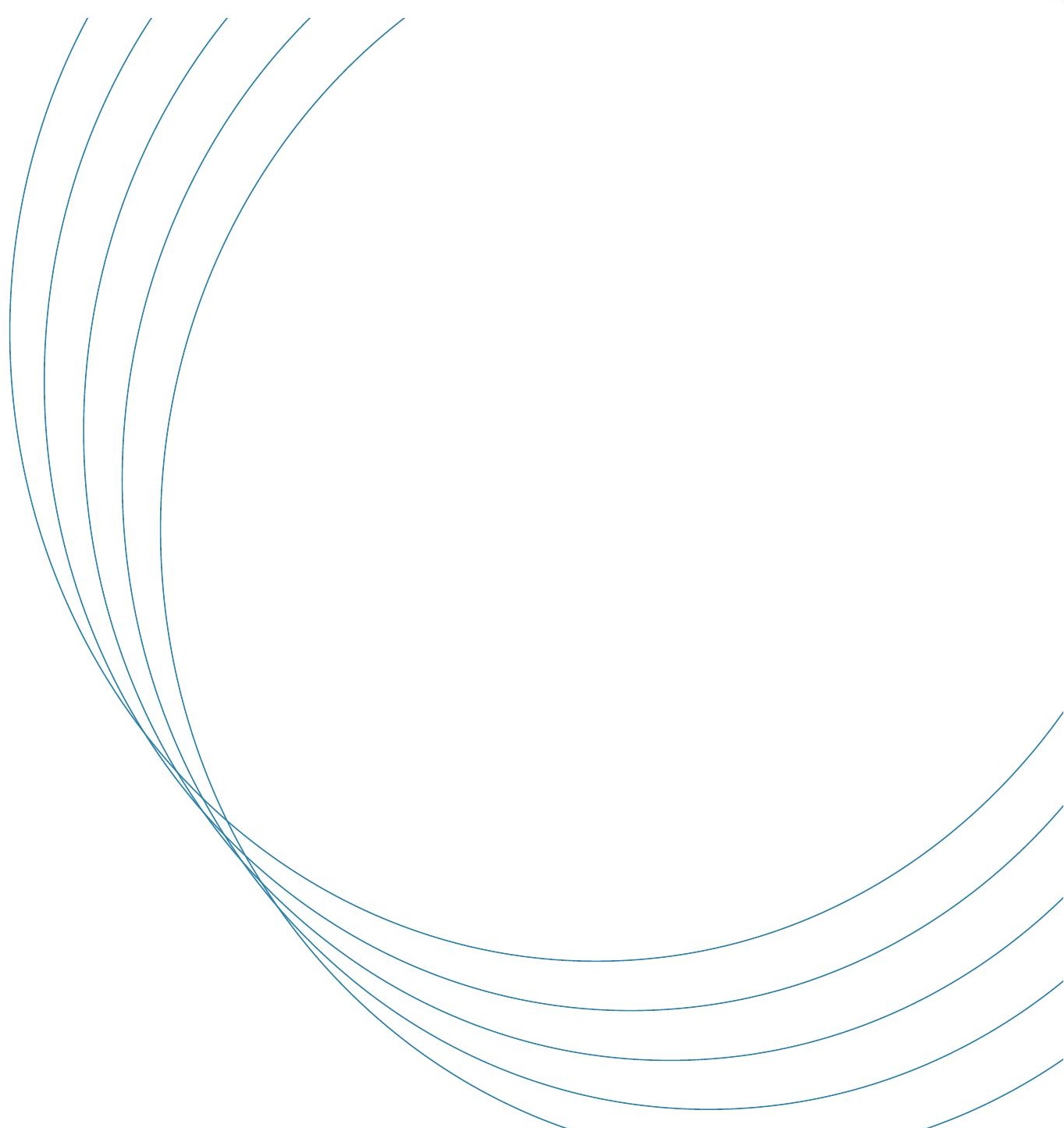




\section{Les contextes physiques d'élévation du niveau}

de la mer

Cette Étude prospective ne porte pas sur le phénomène d'élévation du niveau de la mer lui-même, mais sur les conséquences de cette élévation. Cependant, ce phénomène physique est un élément structurant de la construction des scénarios. Il convient donc de bien le comprendre afin de pouvoir en analyser les conséquences à 2100 . Il faut commencer par définir les contextes physiques possibles d'élévation de la mer à l'horizon 2100 les plus représentatifs et les plus plausibles. Bien des questions doivent être traitées. Quelle amplitude ? Quelle vitesse d'évolution ? Quelles causes et quelle variabilité selon les différentes localisations géographiques?

La problématique de la projection du niveau de la mer à moyen et long terme mobilise de nombreuses disciplines comme la paléoclimatologie, la glaciologie, la physique de l'atmosphère, l'océanographie physique, chimique et biologique, l'hydrologie, l'écologie, la géomorphologie..., et aussi diverses sciences sociales et économiques comme la géopolitique, la sociologie, l'économie, etc. Ce phénomène de hausse de la mer est étudié depuis des décennies et les moyens alloués à la recherche ont été accrus depuis que les estimations ont donné des chiffres inquiétants en termes de conséquences sur les activités humaines (Church et al., 2011, Clark et al., 2013).

Les connaissances accumulées à ce jour permettent d'établir plusieurs assertions bien documentées même si des controverses subsistent sur certains points notamment en matière de vitesse d'évolution et de chaînes de causalité :

- La mer monte en moyenne de $3 \mathrm{~mm} / \mathrm{an}$ depuis 1993 ;

- ce phénomène est en accélération ;

- ce mouvement n'est pas uniforme, ni dans le temps, ni dans l'espace ;

- on identifie assez bien les causes et leur importance respective ;

- mais on ne sait pas bien ce qui se passe à l'échelle locale.

L'analyse des composantes de la montée du niveau de la mer actuelle établit que trois facteurs jouent un rôle majeur dans la montée des eaux (Church et al., 2019). Ce sont :

- la fonte des glaces des calottes polaires, notamment au Groenland (contribution multipliée par 3 en 20 ans) ;

- le gonflement dû à la dilatation de l'eau des océans sous l'effet du réchauffement (effet stérique) ;

- la fonte des glaciers terrestres dans les régions montagneuses.

Ces trois facteurs contribuent grosso modo à parts égales à l'élévation du niveau de la mer. 
Le bilan énergétique déséquilibré de la terre (énergie reçue supérieure à l'énergie réémise) de + 0,5 à $1 \mathrm{~W}$ par mètre carré, lié à l'effet de serre explique l'évolution de ces trois facteurs. Cet excès de chaleur issu des activités humaines est accumulé essentiellement dans l'océan qui, de ce fait, se dilate. Une partie de l'énergie contribue à la fonte des glaces. Depuis 1900, les marégraphes (qui mesurent des valeurs relatives par rapport au socle rocheux) révèlent une hausse moyenne de 1,2 à 1,9 mm/an. Plus récentes, les mesures altimétriques, qui mesurent un niveau marin absolu par satellite, sont plus précises, et donnent 3,0 mm/an + /- 0,4 mm/an depuis 1993.

L'élévation du niveau de la mer peut localement être amplifiée par un affaissement de la surface de la croûte terrestre appelé subsidence, lié le plus souvent à des mouvements tectoniques, mais aussi aux activités humaines. À l'inverse, elle peut être atténuée par la remontée de la croûte terrestre débarrassée du poids de la glace.

La paléoclimatologie montre que le niveau de la mer a beaucoup varié dans le passé et à des vitesses parfois élevées. Toutefois, l'activité anthropique actuelle ne peut être rapprochée d'aucun processus antérieur en raison de la pluralité et de la simultanéité de ses actions : émissions massives de gaz issus de la combustion de ressources fossiles et vivantes (forêts), artificialisation de grands espaces, érosion de la biodiversité et destruction d'écosystèmes à forte capacité de résilience...

Le bilan global, construit par le GIEC à partir de la compilation des travaux des grands centres de recherche mondiaux et de nombreux modèles, est celui d'une courbe nette d'accroissement du niveau de la mer, avec des périodes inexpliquées de pause (notamment, 1984-1989) et d'accélération (ainsi, 1994-2004) et des projections moyennes d'environ $43 \mathrm{~cm}$ à $84 \mathrm{~cm}$ de hausse pour la fin du siècle (Oppenheimer et al., 2019). Les valeurs extrêmes, selon les bilans thermiques envisagés, varient de $29 \mathrm{~cm}$ à $110 \mathrm{~cm}$. Si l'on essaye de prendre en compte les facteurs, encore aujourd'hui mal documentés, les marges d'incertitude et l'accélération en cours du phénomène, notamment sur les calottes glaciaires en Arctique et surtout en Antarctique, ainsi que la probabilité élevée de suivre un des deux scénarios de réchauffement climatique les plus forts du GIEC (RCP 6.0 ou RCP 8.5) dans les trois prochaines décennies au moins, les hypothèses de montée du niveau de la mer doivent être réévaluées à la hausse (Bamber et al., 2019, Jevrejeva et al., 2019 ; Stammer et al., 2019 ; Oppenheimer et al., 2019).

De fait, dès 2012, à la demande du U.S. Climate Change Science Program, la NOAA a conduit une revue de la recherche et a conclu avec un haut degré de confiance (plus de $90 \%$ ) que la hausse du niveau de la mer se situerait entre 0,2 et $2 \mathrm{~m}$ en 2100 (Parris et al., 2012). Par ailleurs, depuis une dizaine d'années, on observe un accroissement de la contribution des calottes glaciaires du Groenland et de l'ouest de l'Antarctique à la hausse du niveau de la mer (Pritchard et al., 2012 ; Mouginot et al., 2014 ; IMBIE team 2018). L'accélération de la fonte des glaces polaires, notamment en raison d'une instabilité croissante des glaciers se déversant dans la mer en Antarctique, phénomène encore mal documenté, justifierait d'envisager le dépassement des projections probables du GIEC (Kopp et al., 2017 ; Le Bars et al., 2017). Ainsi, dans une hypothèse de forte élévation 
de la température moyenne mondiale, et de fonte d'une partie significative des calottes polaires, l'élévation totale pourrait atteindre $2 \mathrm{~m}$ en 2100, voire plus (Foster and Rohling, 2013 ; DeConto et Pollard, 2016 ; Bamber et al., 2019). Compte tenu de ces éléments et de la volonté d'élaborer des scénarios plausibles mais contrastés pour stimuler la réflexion trois hypothèses d'élévation du niveau de la mer en 2100 ont été retenues, soit $+50 \mathrm{~cm}$, $+100 \mathrm{~cm}$ et $+200 \mathrm{~cm}$ pour cette étude.

Par ailleurs, il faut souligner que la valeur absolue de l'élévation du niveau de la mer en 2100 compte moins que la dynamique en cours à cette date. Ainsi, une valeur de $+2 \mathrm{~m}$ en 2100 mais avec une dynamique stabilisée en palier ou en décélération est moins alarmante qu'une valeur de $+1,5 \mathrm{~m}$, mais avec une vitesse de montée des eaux forte, ou pire, toujours en accélération.

Dernier élément important à prendre en compte, la fréquence et l'intensité des événements météorologiques extrêmes (EvEx). Elles sont liées à l'évolution globale du climat avec des quantités croissantes d'énergie thermique à dissiper notamment en mer (vents, vagues, tempêtes, cyclones...) et sur les côtes. Le littoral est donc potentiellement de plus en plus exposé à la submersion marine lors de ces événements alors même que l'on observe à l'échelle mondiale une anthropisation croissante des régions côtières (Wong et al., 2014 ; Leal Filho, 2015). La vulnérabilité des habitats, des infrastructures et des ressources s'accroît donc en proportion de cette évolution. Pour fixer le cadre global de changement climatique qui pourrait s'imposer aux scénarios de l'étude, quatre contextes physiques en 2100 ont été déterminés sur la base de trois paramètres : la hausse moyenne du niveau de la mer par rapport au niveau actuel (2020), la vitesse d'élévation du niveau de la mer en 2100 (forte ou faible), reprenant les trois valeurs de $+50 \mathrm{~cm},+100 \mathrm{~cm} \mathrm{et}+200 \mathrm{~cm}$, et enfin l'intensité et la fréquence des événements météorologiques extrêmes (EvEx).

Les hypothèses d'élévation du niveau de la mer sont logiquement corrélées aux scénarios de forçage radiatif issus des travaux du GIEC (voir tableau 1). On considère ici que l'état physique de type "modéré » n'est compatible qu'avec les deux scénarios les plus optimistes du GIEC (RCP de 2.6 ou 4.5). Celui de type « sérieux » devrait être observé pour des RCP de 4.5 ou 6.o. Enfin, les états physiques qualifiés de « grave » ou « extrême » ne peuvent apparaître que dans les RCP 6.o ou 8.5 (De Conto \& Pollard, 2016). Il convient, naturellement, de nuancer cette répartition car tous les niveaux intermédiaires peuvent être projetés, et observés. Mais le choix de contextes physiques contrastés est indispensable pour construire des scénarios prospectifs eux-mêmes contrastés.

Il est peu plausible que l'on atteigne un ou deux mètres de hausse si la vitesse d'élévation du niveau de la mer est faible en 2100. En conséquence, cette situation est trop peu vraisemblable pour que l'on prévoie des états physiques dans ces deux cas. De même la stabilisation du niveau de la mer en 2100 n'a pas été envisagée comme une hypothèse plausible. La maîtrise de la hausse du niveau de la mer dépend de la maîtrise du réchauffement climatique et suppose donc une coordination mondiale, car il s'agit d'atténuer des phénomènes globaux. Aux échelles locales, on observe de fortes différences en termes d'exposition des zones littorales et d'effets (liées aux vulnérabilités 
spécifiques), ainsi que d'inégales capacités d'adaptation selon les territoires et les États (Rocle et al. 2020). Les conséquences environnementales, sociales et économiques vont donc dépendre de l'ampleur du phénomène, des usages actuels et futurs des espaces littoraux considérés et surtout de la capacité d'anticipation, de réaction et de coordination des instances de gouvernance.

Tableau 1. Caractéristiques des quatre états physiques potentiels concernant le niveau de la mer à 2100

$$
\begin{aligned}
& \text { Vitesse d'élévation du niveau } \\
& \text { de la mer en } 2100 \\
& \text { Hausse du niveau de la mer } \\
& \text { par rapport au niveau de } 2020 \\
& \text { (ordre de grandeur, en } \mathrm{cm} \text { ) }
\end{aligned}
$$

Faible

Forte

\begin{tabular}{lll}
\hline 50 & $\begin{array}{l}\text { MODÉRÉ } \\
\text { (EvEx un peu plus forts) } \\
(\text { RCP 2.6 - 4.5) }\end{array}$ & $\begin{array}{l}\text { SÉRIEUX } \\
\text { (EvEx un peu plus forts) } \\
\text { (RCP 4.5 - 6.0) }\end{array}$ \\
\hline 100 & & $\begin{array}{l}\text { GRAVE } \\
\text { (EvEx plus forts) } \\
\text { (RCP 8.5) }\end{array}$ \\
\hline 200 & EXTRÊME \\
& (EvEx beaucoup plus \\
& forts) \\
& (RCP 8.5)
\end{tabular}




\section{Les trois familles de scénarios}

\section{Le processus d'élaboration}

CetTE PROSPECTIVE S'INTÉRESSE à la façon dont l'ensemble des zones littorales pourraient à l'échelle mondiale être affectées par les effets de la hausse du niveau de la mer en 2100 (voir l'annexe 1 pour une présentation de la méthode). La démarche prend en compte les actions d'anticipation qui pourraient être mises en œuvre sur les littoraux afin de prévenir les conséquences de la hausse du niveau de la mer. Elle s’intéresse aussi aux dynamiques qui déterminent le changement climatique et la hausse du niveau de la mer, sachant que ceux-ci sont liés à de multiples facteurs comme les choix de matière de gouvernance, d'économie, de développement, d'aménagement du territoire...

Le travail de prospective a utilisé une approche systémique, en considérant deux blocs de facteurs déterminants. D’un côté, un système littoral qui est constitué par des populations, des organisations urbaines, des infrastructures, des activités économiques, un environnement et des ressources naturelles, l'agriculture et des structures de gouvernance. De l'autre, un contexte global regroupant les facteurs majeurs externes à la zone littorale qui déterminent les évolutions à long terme des émissions de gaz à effet de serre : la croissance économique mondiale, l'évolution des filières énergétiques, la conscience de l'enjeu climatique et la gouvernance globale.

Pour chacun des facteurs déterminants du système, plusieurs hypothèses d'évolution à l'horizon 2050 ont été construites sur la base de jugements d'experts, en s'appuyant sur trois types de matériaux : l'analyse des tendances passées, la détection des signaux faibles dans le présent et l'identification des ruptures possibles à l'avenir. Par exemple, en ce qui concerne le nombre de personnes habitant dans la zone littorale, donc potentiellement exposées à des risques, quatre hypothèses d'évolution à 2100 ont été élaborées : (1) un retrait progressif, (2) une stabilité (malgré une population mondiale en croissance), (3) un accroissement progressif avec des densités conservées par région, (4) une croissance démographique couplée à une concentration des populations au sein des villes littorales. Le tableau 2 présente l'ensemble des hypothèses qui ont été formulées (voir Tableau 2 : Tableau morphologique complet).

Les scénarios ont été construits en combinant les hypothèses d'évolution des différents facteurs déterminant le système littoral et le contexte global jusqu'à 2100. En sélectionnant des combinaisons cohérentes et plausibles d'hypothèses, 17 scénarios ont été élaborés dans un premier temps. Ils ont ensuite été regroupés et synthétisés en 8 scénarios qui décrivent les avenirs contrastés des systèmes littoraux face à la hausse du niveau de la mer. L'analyse des scénarios a fait émerger trois "familles » dont la ligne de partage tient avant tout à la position de la gouvernance, première variable motrice en matière de prospective environnementale (Lacroix et al., 2019). 
La première famille part d'un « déni » initial, conscient ou inconscient, du changement global en cours. Elle compte trois scénarios distincts. Dans le premier, l'attitude de déni domine pendant des décennies puis, suite à une prise de conscience globale et rapide des coûts comme des risques, elle fait place à une réaction forte, mais qui reste trop tardive pour être efficace (D1). Dans le second, le choix d'une adaptation lente et sans coordination, conduit à un abandon progressif du littoral (D2). Enfin, dans le troisième, l'attitude de déni persiste dans le temps, ce qui conduit inéluctablement à un scénario de passivité pour la gouvernance et de catastrophe pour les littoraux (D3).

La deuxième famille, composée d'un seul scénario, décrit une absence persistante de coordination des politiques d'atténuation à l'échelle mondiale malgré l'ampleur croissante des menaces, et d'une grande hétérogénéité d'action aux échelles territoriale et nationale. Les adaptations, comme les politiques d'atténuation, restent à l'échelle des États ou d'alliances régionales, ce qui est insuffisant pour freiner le changement climatique et ses effets. Ces politiques fragmentées, même si elles sont localement vigoureuses, ne permettent pas de répondre efficacement aux enjeux d'adaptation des littoraux (F).

La troisième famille part d'une volonté d'adaptation des littoraux, qui est plus ou moins couplée à une politique d'atténuation des émissions de gaz à effet de serre. Quatre scénarios structurent cette famille en fonction de l'ampleur de l'effort d'adaptation nécessaire pour faire face à la hausse du niveau de la mer. Dans le premier, une politique d'atténuation ambitieuse coordonnée à l'échelle mondiale permet d'aboutir en 2050 à une situation de maîtrise climatique, ce qui permet de rester à un effort limité en matière d'adaptation littorale (A1). Dans le second, les États, les entreprises et les sociétés, prenant la mesure des changements en cours et de leurs causes, mettent en œuvre des objectifs de sobriété, ce qui permet d'anticiper les risques de la non-action, de renforcer les efforts d'atténuation et de réagir à temps pour adapter les littoraux ( $\left.A_{3}\right)$. Dans un troisième, la réaction en termes d'atténuation a lieu plus tard au cours du siècle ; les politiques d'adaptation littorales mises en œuvre, variables selon les pays et les situations sur les zones côtières, se focalisent en priorité sur les villes littorales où se concentrent populations, activités et richesses patrimoniales pour l'homme (A2). Dans un quatrième, les logiques d'adaptation littorale restent longtemps prédominantes et efficaces en l'absence de tout effort sérieux d'atténuation jusqu'à ce que, sous la contrainte des phénomènes majeurs liés à la hausse du niveau de la mer, l'atténuation devienne à son tour une priorité, mais trop tardivement pour avoir un effet significatif (A4).

\section{Description des scénarios à 2100}

\section{Les scénarios du déni du changement climatique ou du déni de l'élévation du niveau de la mer}

\section{D1 : Du déni à la réaction}

Les enjeux du changement climatique et les risques liés à l'élévation du niveau de la mer font l'objet dans un premier temps d'un déni de la part de la société et des gouvernants qui conduit à une passivité générale et à une absence complète d'anticipation 
Tableau 2. Tableau morphologique complet

Composante

\begin{tabular}{cl} 
& $\begin{array}{l}\text { P1- Part de la population } \\
\text { exposée }\end{array}$ \\
\cline { 2 - 3 } $\begin{array}{c}\text { Population } \\
\text { littorale }\end{array}$ & $\begin{array}{l}\text { P2 - Migrations internes } \\
\text { et internationales }\end{array}$ \\
\cline { 2 - 3 } & $\begin{array}{l}\text { P3 - Degré de vulnérabilité } \\
\text { sanitaire des populations }\end{array}$ \\
\hline \multirow{3}{*}{$\begin{array}{c}\text { Urbanisme et } \\
\text { infrastructures }\end{array}$} & $\begin{array}{l}\text { U1 - Dynamiques urbaines } \\
\text { sur la zone côtière }\end{array}$ \\
\cline { 2 - 3 } & $\begin{array}{l}\text { U2 - Niveau de résilience des } \\
\text { infrastructures }\end{array}$ \\
\cline { 2 - 3 } & $\begin{array}{l}\text { U3 - Adaptation des zones } \\
\text { littorales exposées }\end{array}$
\end{tabular}

EN1 - État des ressources en eau douce (Quantité et Qualité)

Environnement

et ressources

naturelles

et alimentation $\mathrm{AA}_{3}$-Poids de l'aquaculture

Économie

\section{EN2 - État des sols}

(salinisation, érosion...)

$\mathrm{EN}_{3}$ - Dynamiques des écosystèmes littoraux et côtiers (habitats, biodiversité)

EN4 - Modification du trait de côte

\begin{tabular}{ll}
\hline$A A 1-$ Disponibilité en terres \\
agricoles en zone côtière \\
\cline { 2 - 2 } AA2 - Systèmes de production \\
agricoles en zone côtière
\end{tabular}
et de la pêche

$\mathrm{AA}_{4}$ - Sécurité alimentaire
Hypothèse 1

Décroissance, car retrait progressif de la population des zones côtières

Des départs échelonnés, au fil de la montée progressive des eaux

Un bon accès aux

infrastructures de santé limite la vulnérabilité des populations

Forte urbanisation et mégavilles littorales

Vulnérabilité forte (résilience faible)

Résister à l'élévation du niveau de la mer (priorité aux digues)

Maîtrise de l'exploitation et de

l'usage des ressources

Salinisation et pollution faibles

Translation et/ou modification

sans altération des fonctions

écosystémiques

Érosion modérée et recul marginal

\section{Forte}

Forte urbanisation et dispersion urbaine

Stabilité de la partie en zone côtière (malgré une population mondiale en croissance) Les évacuations s'accélèrent, certaines villes importantes sont touchées Les zones côtières ont une forte vulnérabilité sanitaire Vulnérabilité moyenne (résilience moyenne) Faire avec l'élévation par une adaptation progressive Dégradation progressive, altération des fonctions écologiques

Salinisation et pollution modérées à moyennes Adaptation/modification in situ, altération des fonctions écosystèmiques Érosion moyenne et hétérogène ; recul moyen localisé prévisible

Réduction de plus de la moitié Protection efficace des terres des terres agricoles agricoles

Adaptation des espèces Substitution progressive cultivées et des pratiques des cultures par l'élevage agronomiques

Maintien des apports via pêche Diminution des apports de et aquaculture durables la pêche et aquaculture stable

Réduction de l'accès économique aux productions agricoles

\begin{tabular}{cl}
\hline \multirow{2}{*}{$\begin{array}{c}\text { Économie } \\
\text { littorale }\end{array}$} & $E C$ - Économie littorale \\
\cline { 2 - 2 } & $\begin{array}{l}\mathrm{EC} 2-\text { Solidarité et adapatation } \\
\text { aux situations de crises }\end{array}$
\end{tabular}

Multiplication des formes de valorisation économique in situ Perturbations ou ruptures ponctuelles de l'accès Brutalisation » des rapports Solidarité à tous les niveaux sociaux

G1 - Prise de conscience des risques littoraux Appropriation des enjeux de la Déni montée des eaux

Gouvernance G2 - Réactivité et degré

littorale d'engagement des acteurs

G3 - Niveau (local et global) Élevé, et aux deux niveaux: de coordination/mutualisation
Passivité sans implication Implication minimale

Élevé, et aux deux niveaux: Inexistant ; règle du « chacun local et mondial pour soi » 
Hypothèse 3

Accroissement progressif de la population en zone côtière
Hypothèse 4

Hypothèse 5

Forte croissance conjuguée

à des migrations au sein/vers

des mégalopoles côtières

Crises répétées engendrant des exodes massifs

La prolifération des mégalopoles

côtières augmente encore

la vulnérabilité sanitaire

Faible urbanisation et villes en réseau

avec arrière-pays

Vulnérabilité maîtrisée (résilience

développée)

Organiser le retrait par un

Absence de stratégie spécifique

changement structurel

Nécessité d'importer de l'eau de

zones extérieures vers le littoral

Salinisation, pollution et

imperméabilisation fortes

Forte altération allant jusqu'à

la disparition d'écosystèmes

Érosion accélérée hétérogène ; recul Recul marqué généralisé

fort mais localisé

Quasi disparition des terres agricoles

en zone côtière

Synergies des systèmes agricoles

et aquacoles

Accroissement des apports via une

aquaculture diversifiée

Réduction de la diversité de Amélioration par la diversification des

l'alimentation

sources d'approvisionnement

\begin{tabular}{lll}
$\begin{array}{l}\text { Repli stratégique planifié et relance } \\
\text { via l'arrière-pays }\end{array}$ & $\begin{array}{l}\text { Économie du flottant, de } \\
\text { l'aménagement « avec la mer » }\end{array}$ & $\begin{array}{l}\text { Déplacement massif des activités } \\
\text { économiques }\end{array}$ \\
$\begin{array}{l}\text { Deux extrêmes ensemble : cartel } \\
\text { des riches et solidarité des pauvres }\end{array}$ & $\begin{array}{l}\text { Chacun pour soi et stabilité par } \\
\text { redistribution humanitaire }\end{array}$ & $\begin{array}{l}\text { Financiarisation assurantielle et } \\
\text { judiciarisation des tensions }\end{array}$ \\
\hline Prise de conscience de façade & $\begin{array}{l}\text { Clivages et persistance du déni ou } \\
\text { du scepticisme }\end{array}$ & \\
$\begin{array}{l}\text { Réactivité forte et mobilisation } \\
\text { de la majorité des acteurs }\end{array}$ & $\begin{array}{l}\text { Proactivité et implication de tous } \\
\text { les acteurs }\end{array}$ & \\
\hline $\begin{array}{l}\text { Ciblé d'abord sur les, villes cotières } \\
\text { coordonnées en réseau }\end{array}$ & $\begin{array}{l}\text { Élevé, mais sous le contrôle } \\
\text { des multinationales type GAFAM }\end{array}$ & $\begin{array}{l}\text { Fort, piloté par les régions dans } \\
\text { les choix stratégiques }\end{array}$ \\
\hline
\end{tabular}




\section{Tableau 2 (suite)}

\begin{tabular}{|c|c|c|c|}
\hline Composante & Variable & Hypothèse 1 & Hypothèse 2 \\
\hline \multirow{4}{*}{ Contexte global } & $\begin{array}{l}\text { C1 - Croissance économique } \\
\text { globale }\end{array}$ & $\begin{array}{l}\text { Décroissance choisie } \\
\text { (recyclage, sobriété, éducation } \\
\text { écologique...) }\end{array}$ & $\begin{array}{l}\text { Croissance à deux vitesses, } \\
\text { selon les régions du monde }\end{array}$ \\
\hline & $\begin{array}{l}\text { C2 - Bouquet énergétique } \\
\text { (dépendance au carbone) }\end{array}$ & $\begin{array}{l}\text { Synergies de tous les acteurs } \\
-\rightarrow>\text { décarbonation de } \\
\text { l'économie }\end{array}$ & $\begin{array}{l}\text { Fragmentation de la production } \\
\text { et de la consommation } \\
\text { énergétique }\end{array}$ \\
\hline & $\begin{array}{l}\text { C3 - Prise de conscience } \\
\text { des enjeux du changement } \\
\text { climatique }\end{array}$ & $\begin{array}{l}\text { Appropriation progressive } \\
\text { et partagée }\end{array}$ & Déni majoritaire et persistant \\
\hline & $\begin{array}{l}\mathrm{C}_{4} \text { - Gouvernance géopolitique } \\
\text { globale }\end{array}$ & Chaos généralisé & $\begin{array}{l}\text { Fragmentation dans un monde } \\
\text { mutipolaire turbulent et } \\
\text { contrasté }\end{array}$ \\
\hline
\end{tabular}

et d'implication sur ces enjeux. Vers le milieu du siècle, sous la pression des conséquences du changement climatique, ce déni initial cède la place à des stratégies simultanées d'adaptation littorale et d'atténuation du changement climatique, qui limitent la vulnérabilité littorale, mais conduisent néanmoins à un état physique « grave » en 2100. L'urbanisation du littoral se poursuit et la population continue de s'accroître dans la zone littorale en se concentrant dans des mégapoles. L'absence de stratégie d'adaptation des zones littorales les plus exposées contribue à accroître la vulnérabilité des infrastructures et des réseaux qui n'ont pas été conçus en tenant compte de ces nouveaux risques. Les ressources en eau douce se dégradent dans les régions côtières sous l'effet cumulé d'une salinisation progressive des nappes phréatiques et de la pollution lors des événements extrêmes de submersion ou d'inondation, de plus en plus fréquents et violents, alors même que les besoins en eau potable augmentent. Cette dégradation d'une ressource vitale entraîne la multiplication des conflits d'usage.

Les services écosystémiques sont altérés et des écosystèmes côtiers entiers disparaissent sous la pression de l'urbanisation du littoral et de l'élévation du niveau de la mer, ce qui augmente encore la vulnérabilité des côtes. Le trait de côte recule dès lors que le littoral de faible élévation, ce qui conduit conduisant à un repli contraint, anarchique et par à-coups de l'économie littorale.

Les sols agricoles disparaissent peu à peu en zone côtière sous les effets cumulés de la salinisation, de l'imperméabilisation et de la pollution croissante des sols. Ceci conduit à une substitution des cultures par de l'élevage hors sol et de l'aquaculture. Les apports de la pêche et de l'aquaculture augmentent, mais la diversité de l'alimentation se réduit. La croissance économique globale en dents de scie dans un monde multipolaire et turbulent se traduit par une politique énergétique de court terme, fluctuante dans ses priorités et 


\begin{tabular}{|c|c|c|}
\hline Hypothèse 3 & Hypothèse 4 & Hypothèse 5 \\
\hline Croissance en " stop and go » & $\begin{array}{l}\text { Décroissance subie ; chaos politique } \\
\text { et économique }\end{array}$ & \\
\hline $\begin{array}{l}\text { Chaos énergétique et priorité } \\
\text { à la sécurité énergétique nationale }\end{array}$ & $\begin{array}{l}\text { Priorité à l'autonomie énergétique } \\
\text { (sources locales) }\end{array}$ & \\
\hline Prise de conscience de façade & $\begin{array}{l}\text { Clivages marqués et crispation } \\
\text { des positions }\end{array}$ & \\
\hline $\begin{array}{l}\text { Généralisation d'un ordre } \\
\text { cybernétique fondé sur l'IA } \\
\text { (l'intelligence artificielle) }\end{array}$ & $\begin{array}{l}\text { Domination partagée entre } \\
\text { deux super puissances : Chine } \\
\text { et États-Unis }\end{array}$ & $\begin{array}{l}\text { Émergence de la gestion } \\
\text { collective des biens communs }\end{array}$ \\
\hline
\end{tabular}

toujours fondée principalement sur des ressources fossiles. Les technologies de l'exploitation des ressources renouvelables se développent mais sans politique mondiale intégrée. Au milieu du siècle, face à la constatation de l'ampleur de l'élévation du niveau de la mer, d'événements extrêmes plus fréquents et plus violents et de leurs conséquences, s'amorce un retrait progressif de la population des zones côtières, en raison de leur forte vulnérabilité sanitaire. On assiste à des départs échelonnés au fil de la montée progressive des eaux. Ces phénomènes de migration, et les conflits qu'ils génèrent, contraignent les États à réagir et à s'impliquer. Ceux-ci mettent alors en place des mécanismes de redistribution humanitaire qui s'avèrent de plus en plus coûteux. Cela conduit à une prise de conscience progressive de la société et des décideurs de la réalité et de la dangerosité des phénomènes en cours. Les scientifiques sont plus écoutés par une opinion publique qui prend conscience de la nécessité d'une réaction collective face aux risques. Les pouvoirs publics, poussés à agir à la fois sur les effets et sur les causes, mettent en place des stratégies d'adaptation littorale et d'atténuation du changement climatique, mais trop tardives pour en voir les effets avant le milieu du siècle suivant.

\section{D2 : Abandon progressif du littoral}

D’abord abandonnée par les acteurs économiques dominants et les pouvoirs publics, la zone littorale ne bénéficient que tardivement d'action de défense (2080). Les décideurs politiques ne consentent à défendre des zones littorales abandonnées aux populations pauvres que pour prévenir ou réduire les migrations massives de populations, mais sans agir sur l'atténuation du changement climatique, ce qui conduit inexorablement à un état physique « extrême » en 2100.

Dominé par quelques superpuissances, le monde connaît une croissance économique inégalitaire, où les tensions entre une forte croissance économique pour quelques oligopoles créateurs de richesses et une faible croissance pour la majorité de l'humanité 
s'accentuent. Le «bottom billion » ou le milliard de personnes les plus pauvres, double en 30 ans). Entre ces deux extrêmes, on observe la paupérisation progressive des classes moyennes. Globalement, la richesse mondiale augmente mais cet enrichissement est capté au profit des classes les plus aisées. Le désordre énergétique qui accompagne ces disparités conduit au repli sur la sécurité énergétique et l'utilisation en priorité des ressources énergétiques locales, y compris fossiles, plutôt que la recherche d'énergies décarbonées. La montée progressive du niveau de la mer provoque une dégradation des écosystèmes littoraux avec une salinisation et une pollution des sols. Face au recul marqué du trait de côte et à la disparition de plus de la moitié des sols agricoles en zone littorale, la classe économique dominante, lucide sur les évolutions climatiques et littorales à venir, organise le repli stratégique des activités économiques vers l'arrière-pays et abandonne la zone côtière aux classes pauvres, mal informées, et ayant peu d'accès au foncier. Elles y développent des activités de subsistance. De fréquentes perturbations, voire des ruptures ponctuelles, des approvisionnements alimentaires apparaissent malgré l'accroissement des apports de l'aquaculture. La salinisation des nappes phréatiques côtières impose de recourir à un transfert massif de ressources hydriques extérieures vers la zone littorale, accroissant encore la dépendance des populations littorales. Malgré une urbanisation littorale limitée, mais anarchique, l'imperméabilisation des sols reste importante et les écosystèmes littoraux continuent à se dégrader, entraînant la perte des services écosystémiques associés.

Face au manque d'anticipation et à la défaillance des services publics, chacun s'organise et s'adapte comme il peut. Ceux qui en ont les moyens fuient la zone littorale et rejoignent l'arrière-pays. Les évacuations s'accélèrent après chaque événement climatique extrême, créant des conflits de plus en plus violents, mais également des actions humanitaires ponctuelles soutenues à la fois par l'État et les acteurs économiques dominants pour maintenir les populations pauvres en place sur le littoral. À la fin du siècle, certaines villes littorales importantes sont gravement affectées. Ceux qui restent s'organisent au mieux, mais peinent au début à mobiliser des aides au-delà des régions qui partagent les mêmes épreuves dans une communauté de destin. Cependant, de mieux en mieux coordonnées en réseau, ces villes parviennent à convaincre l'État, les régions et les acteurs économiques dominants de la nécessité d'agir pour sauver ce qui peut l'être, éviter les désastres humains lors de tempêtes et freiner les migrations vers les villes de l'intérieur. Elles organisent des formes de redistribution via l'État afin de maintenir les populations pauvres dans les zones littorales que l'on peut protéger à moindre coût et limitent la vulnérabilité sanitaire des populations en adaptant a minima les infrastructures locales. Cette solution apparaît moins coûteuse et plus directement efficace que d'agir sur l'atténuation du changement climatique, ambition hors de portée d'un monde en repli.

\section{D3 : Passivité}

Le déni de l'accélération du changement climatique et de la montée du niveau de la mer se poursuit jusqu'en 2100. La passivité et l'absence d'anticipation qu'engendre ce déni 
conduisent à une forte vulnérabilité des régions côtières dans un contexte physique devenu « extrême ».

Les gouvernants, comme la majorité de la population, restent dans le déni, encouragés par les postures idéologiques défendues par des États dont la puissance économique pèse au niveau mondial. Ces États dominants freinent les États, minoritaires, qui souhaiteraient mettre en œuvre des solutions d'atténuation, les jeux économiques les mettant en défaut de moyens, voire en faillite. Sans vision d'avenir, les gouvernants gèrent le quotidien et les urgences. Dans un contexte global d'absence de coordination entre les acteurs, de passivité quasi générale face aux menaces croissantes sur le littoral et de manque de décision sur le long terme, c'est le règne du " chacun pour soi ». Les crises chroniques systémiques qui en résultent conduisent au repli de chaque État sur sa propre sécurité et prioritairement à l'utilisation des ressources énergétiques locales, y compris fossiles, plutôt que la recherche d'énergies décarbonées.

Sans stratégie ni coordination, l'accroissement anarchique des populations dans les zones côtières, principalement dans les villes, se poursuit. Du fait de la répétition des crises liées aux conséquences des événements climatiques extrêmes, de plus en plus intenses, on assiste localement et par à-coups à des exodes massifs. L'absence d'anticipation et de stratégie d'adaptation, face à la montée du niveau de la mer, et la forte urbanisation littorale, du fait du développement de mégapoles côtières, crée les conditions d'une vulnérabilité accrue pour les infrastructures. L'économie littorale s'en trouve perturbée et doit entamer un repli par à-coups au gré des événements climatiques extrêmes de plus en plus fréquents. Les classes pauvres ne pouvant compter sur aucune solidarité, ni aucune forme de mutualisation, la brutalisation des rapports sociaux est inévitable.

Cette anarchie urbaine littorale et l'imperméabilisation des sols qui l'accompagne se traduisent sur l'environnement et les ressources par (1) une dégradation progressive des écosystèmes littoraux et de leurs fonctions écologiques, (2) des phénomènes de salinisation et/ou de pollution des ressources en eau douce et des sols liée à des submersions et des inondations de plus en plus fréquentes et étendues. Certains écosystèmes littoraux (les mangroves, par exemple) disparaissent sous l'effet de la hausse du niveau de la mer, ce qui accélère le recul du trait de côte.

L'agriculture et l'alimentation pâtissent des conséquences de ce déni et du laisser-faire avec une réduction de moitié des terres agricoles dans les régions littorales. L'élevage hors-sol remplace les cultures. Le poids de la pêche est irrégulier et peut varier fortement selon des conditions environnementales. L'aquaculture se diversifie, notamment en milieu saumâtre à l'intérieur des terres. Dans tous les cas, les difficultés d'approvisionnement alimentaire rendent plus vulnérables et dépendantes les populations côtières soumises à des perturbations de l'accès aux productions agricoles, ainsi qu'à une réduction de la diversité de l'alimentation. Cette posture de déni mène à terme à des situations de crise dans tous les domaines de la gestion territoriale comme de la sécurité des biens et des personnes. Les États défaillants perdent toute crédibilité vis-à-vis des populations dont ils sont censés assurer la sécurité et favoriser le développement. 


\section{Le scénario de la fragmentation de la gouvernance internationale}

\section{F : Fragmentation persistante}

Face à une dégradation globale de l'environnement et notamment des littoraux et des fonctions écologiques associées, les États développent des stratégies d'autonomie nationale. Les différences de moyens financiers et technologiques entre États et l'absence de solidarité internationale conduisent à de fortes divergences entre les grandes régions mondiales, voire entre provinces d'un même État quand leurs situations sont contrastées. La logique politique dominante, stable au sein de régimes souvent autoritaires, est celle de la « défense des intérêts nationaux d'abord ». Ce monde, fragmenté et turbulent, fait coexister une grande variété de situations avec aux extrêmes, d'un côté, des États riches disposant de ressources et de technologies suffisantes pour leur permettre de se protéger de la montée des eaux afin de sécuriser patrimoine et ressources et, de l'autre côté, des États pauvres ou faillis qui subissent les contraintes du changement global sans capacité de remédiation ni d'anticipation des conséquences.

Ces trajectoires différentes renforcent une évolution vers un fonctionnement quasi « bipolaire » de la planète, où certains États renforcent sans cesse leurs capacités de protection des populations et des activités en région côtière, tandis que les autres, à l'inverse, subissent les risques climatiques et abandonnent les territoires devenus trop inhospitaliers en termes de sécurité et d'accès aux ressources. Cette évolution laisse peu de place à la collaboration internationale, en particulier à l'atténuation collective du changement climatique. Par conséquent, le contexte physique, déjà " sérieux » en 2050, ne fait qu'empirer dans les décennies suivantes, justifiant de plus en plus les politiques de repli et d'individualisme des États.

Les politiques touchant à l'urbanisation du littoral constituent un exemple emblématique. Ainsi, les États les plus développés mènent de front une politique de lutte contre l'invasion marine (construction et/ou élévation de digues ; zones tampons, habitat flottant...), tout en réduisant l'urbanisation des zones littorales. Les villes restantes s'adaptent aux contraintes en privilégiant les systèmes de gestion : modélisation des risques, alarmes, évacuations préventives... À l'inverse, les États les plus pauvres ne peuvent s'opposer à la prolifération de mégalopoles situées en zones littorales, recueillant notamment les populations rurales chassées par la perte de leurs terres agricoles. Cette évolution accroît la vulnérabilité des populations aux phénomènes naturels et aux crises sanitaires.

Il en va de même en matière de gestion de l'environnement et des ressources (limitation des dégâts d'un côté et dégradation marquée de l'autre) ou de l'accès aux ressources alimentaires : diversification des pratiques et des sources d'approvisionnement d'un côté contre dégradation des terres et crises alimentaires chroniques de l'autre.

L'occurrence croissante d'événements climatiques extrêmes entraîne une augmentation des évacuations temporaires, voire des relocalisations. Certaines villes importantes, y compris des capitales comme Djakarta, Bangkok ou Manille, sont déplacées. Au regard des disparités extrêmes et des crises alimentaires répétées, les déplacements de population 
finissent par évoluer en migrations massives chroniques, très difficiles à réguler au niveau international. Dans un tel contexte, la montée de foyers de violence n'est pas à exclure.

\section{Les scénarios de l'adaptation de la zone littorale}

\section{A1 : Maîtrise climatique}

L'atténuation du changement climatique s'est mise en place de manière précoce et massive, vers 2025, limitant l'élévation du niveau de la mer en 2100. Les acteurs nationaux se sont coordonnés à l'échelle internationale pour une gestion globale des biens communs et surtout ont mis en place des mesures proactives pour arriver à la neutralité carbone avant 2050 par une double stratégie. En premier lieu, l'ensemble des acteurs contribuent à la mise en œuvre d'un vaste programme mondial de décarbonation de l'économie, incluant une transition énergétique basée sur une sortie des énergies fossiles, leur substitution par des énergies renouvelables, de fortes mesures d'efficience énergétique, (découplage du PIB et de la consommation d'énergie), le captage et le stockage du carbone dans les couches géologiques profondes et le renforcement des fonctions des puits de carbone (océan, sols, forêts...).

Face à un changement climatique limité engendrant une faible hausse du niveau de la mer, soit un contexte physique considéré comme " modéré », les stratégies d'adaptation du littoral qui sont mises en œuvre restent des mesures incrémentales basées sur la transformation des villes sans remise en cause du développement urbain. Pour autant, la hausse du niveau de la mer et les événements extrêmes ont ponctuellement des impacts importants sur certains littoraux plus exposés aux aléas.

S'appuyant sur une perception similaire parmi la majorité des acteurs de l'importance des changements globaux et du rôle central du climat, des politiques globales d'atténuation du changement climatique sont mises en place de manière précoce et massive (dès 2025), ce qui a permis de freiner le changement climatique et donc de réduire ses conséquences sur la hausse du niveau de la mer.

Cependant, en raison de leur attractivité continue, les espaces littoraux connaissent une croissance de leur population et un développement rapide de l'urbanisation et de mégavilles, ce qui augmente leur vulnérabilité. Les aménagements du littoral mis en œuvre pour faire face à la montée du niveau de la mer correspondent à des stratégies incrémentales et à une transformation de la ville sur elle-même. Dans certaines zones plus exposées, comme les grands deltas, où les phénomènes de subsidence des sols sont particulièrement marqués, des politiques d'aménagement et de planification urbaines visant un changement transformationnel sont rendues nécessaires afin de déplacer les populations et les infrastructures urbaines vers des zones d'arrière-pays moins exposées aux aléas.

Les espaces agricoles littoraux se réduisent, conséquence de la forte urbanisation. Cependant, les ressources alimentaires se diversifient grâce au maintien de la mondialisation des échanges et au développement local de nouvelles formes d'agriculture et d'aquaculture, plus durables et moins consommatrices d'eau. Un haut niveau de sécurité alimentaire est ainsi maintenu malgré une salinisation progressive d'une partie des terres agricoles. 
La bonne connaissance des mécanismes en jeu, la concertation permanente entre les usagers de l'espace et l'implication de tous les acteurs dans les décisions permettent de maintenir un bon état global des milieux naturels et des ressources afférentes. Cependant, des développements urbains mal régulés peuvent entraîner dans certains pays des altérations des écosystèmes littoraux et de leurs services associés, ce qui mobilise les efforts de la communauté internationale.

\section{A2 : Villes résilientes}

Les grandes villes et les mégapoles, devenues le cœur de l'économie mondiale, jouent un rôle crucial dans l'adaptation à la hausse du niveau de la mer et l'atténuation du changement climatique. La croissance de la population, importante dans les zones littorales, se concentre peu à peu dans des villes côtières du fait de leur attractivité (emplois, services, etc.). Les autres zones littorales sont délaissées en raison de catastrophes récurrentes, sans volonté de protection de la part des autorités. En conséquence, les populations rurales côtières se replient vers des zones d'arrière-pays moins exposées aux aléas, ou vers les métropoles côtières, mieux protégées.

La coordination de ces grandes villes, structurées en grands réseaux régionaux, permet la mise en œuvre des mesures de réduction des émissions de gaz à effet de serre, initiative généralisée par les États à toutes les activités émettrices, ce qui a pour effet de limiter le changement climatique et ses conséquences. L'adaptation du littoral se concentre sur les villes, délaissant les zones littorales de faible densité. Ces villes en réseau, grâce à l'expérience partagée de systèmes d'innovation et d'adaptation, renforcent leur résilience face aux effets du changement climatique, comme les vagues de chaleur, les événements météorologiques marins extrêmes et la hausse du niveau de la mer, grâce, dans un premier temps, à des protections artificielles et naturelles. Ensuite, en s'appuyant sur des technologies numériques (internet des objets, données massives, modélisation, intelligence artificielle...) et sur une ingénierie urbaine innovante, ces villes deviennent capables de freiner les mécanismes de subsidence, de renforcer la sécurité alimentaire, de développer des infrastructures flottantes (ports, aéroports, parcs énergétiques) et surtout d'accroître la résilience des infrastructures énergétiques de communication et de transport. L'atténuation du changement climatique s'organise entre 2030 et 2050, à l'initiative des grandes métropoles mondiales. Elles mettent en œuvre de forts investissements pour la transition énergétique et la réduction des émissions des gaz à effet de serre, ce qui conduit à freiner la vitesse du changement climatique et à restreindre la hausse du niveau de la mer à $1 \mathrm{~m}$ en 2100, même si la hausse perdure. En conséquence, en dehors des régions urbanisées, les zones littorales évoluent vers des situations de vulnérabilité croissante face aux aléas. Le contexte physique général doit alors être considéré comme " sérieux ». Les populations, les activités et les infrastructures se replient sur l'arrière-pays. L'agriculture reste présente, mais les terres agricoles littorales se réduisent sous l'effet conjoint de la salinisation et de l'artificialisation des sols à la périphérie des métropoles. Le trait de côte subit de fortes modifications. De manière générale, les 
impacts négatifs sur les écosystèmes littoraux et côtiers sont importants dans ce scénario, à la fois du fait de l'élévation du niveau de la mer, du retrait de la ligne de côte et de l'artificialisation des sols.

\section{A3 : Sobriété et anticipation}

Face à la menace d'une élévation du niveau de la mer, c'est le choix de la sobriété des modes de vie à l'échelle mondiale qui déclenche l'inversion de la tendance des émissions de gaz à effet de serre, et permet de limiter le changement climatique, à défaut de le maîtriser, avant 2100. Cependant, du fait des dynamiques amorcées et des émissions qui persistent pendant des décennies, l'élévation du niveau de la mer en 2100 reste significative en moyenne par rapport à ce qui était projeté en 2020, ce qui fait que l'on doit parler d'un contexte physique "sérieux ». Mais la vitesse modérée d'élévation du niveau de la mer a permis l'accumulation suffisante de données scientifiques incontestables pour initier un changement des mentalités et des modes de vie. Décideurs et opinions publiques s'approprient aussi l'idée générale que, dans les zones littorales, le repli stratégique coordonné à grande échelle est la meilleure solution. Peu à peu, plus solidaires et mieux coordonnés face à une menace planétaire, les États sont plus efficaces pour minimiser les effets négatifs de l'élévation en adaptant les littoraux et pour anticiper la restructuration territoriale. Priorité est donnée à la restructuration progressive de l'économie littorale afin de planifier un repli stratégique en redéployant les activités sur l'arrière-pays.

La gouvernance mondiale, malgré de fortes disparités d'évolution d'une région à une autre, fait le choix d'une décroissance économique, par la mise en œuvre d'une sobriété plus ou moins bien acceptée des modes de vie, s'appuyant sur des mouvements de citoyens en pays démocratique et sur le triptyque injonction/contrôle/sanction pour les pays dirigistes (Conway et Oreskes, 2014). Les investissements se concentrent alors dans les domaines de la recherche et de l'innovation technologique en vue de l'adaptation littorale. Les sources locales d'énergie sont privilégiées pour atteindre une autonomie énergétique croissante. La gouvernance, qui implique de plus en plus de citoyens structurés en réseaux à multiples échelles, joue un rôle clef dans l'équilibre des ressources et des sociétés.

La population littorale reste stable dans une première phase, avant d'accepter le déplacement vers des zones sûres. En matière d'urbanisation et d'infrastructures, on passe progressivement d'une politique d'ouvrages de protection à l'organisation d'un repli en échelons : dans un premier temps, les ouvrages de protection permettent de sécuriser les terres agricoles et les villes, ce qui donne du temps pour préparer les replis, là où cela s'avère nécessaire, sur plusieurs échelles de temps. Ensuite, la restructuration territoriale, anticipée et modélisée, permet un développement plus durable car mieux pensé : agroforesterie, diversification des productions agricoles en fonction des tendances climatiques locales, ville végétalisée, zones-éponge aux interfaces avec les fleuves et la mer, revalorisation de la biodiversité... autant de domaines générateurs d'innovations et d'emplois.

En matière d'environnement, la concertation permanente à tous les niveaux décisionnels et la détermination des acteurs à diminuer l'exploitation et l'usage des ressources permettent 
de conserver une certaine maîtrise des milieux et des ressources, notamment l'eau. La salinisation et la pollution des sols, l'érosion et le recul du trait de côte restent ainsi modérés.

\section{A4 : Adaptation prioritaire et atténuation tardive}

Depuis 2020, l'adaptation à la hausse du niveau de la mer s'est rapidement mise en place dans toutes les régions littorales du monde, mais les actions en faveur de l'atténuation du changement climatique, tardives, ne se sont développées qu'après 2050. Il en résulte en 2100 un changement climatique marqué et une hausse importante du niveau de la mer. Après 2050, la mise en place de mesures globales de lutte contre le changement climatique (par exemple, une décarbonation de l'économie) permet d'éviter le pire en termes d'élévation du niveau de la mer, en limitant la hausse à $1 \mathrm{~m}$, mais le phénomène reste en accélération, ce qui justifie de considérer le contexte physique global comme " grave ». Jusqu'en 2060, les politiques étatiques poursuivent les tendances observées en 2020. Le monde est fragmenté, en mosaïque, avec une instabilité géopolitique, une croissance économique en dents de scie (notamment, en raison des effets des pandémies), une lente prise de conscience des enjeux du changement climatique, et le maintien de la dominance des énergies fossiles dans la consommation énergétique. Mais en 2050-2060, l'accélération du changement climatique est telle qu'il devient urgent d'agir pour limiter ses impacts et les coûts d'adaptation, devenus exorbitants. Cette prise de conscience débouche sur la mise en place autoritaire d'une coopération mondiale pour la gestion des biens communs et sur une transition énergétique accélérée vers la décarbonation de l'économie.

Anticipant le recul prévisible de leur trait de côte, les régions ont déjà mis en place des stratégies d'adaptation qui permettent de limiter l'impact de la hausse du niveau de la mer. Elles agissent, d'une part, en contrôlant le développement urbain et en stabilisant leur croissance démographique, et, d'autre part, en s'adaptant peu à peu aux risques par des innovations incrémentales et le déplacement des habitations et des populations les plus exposées. Dans ce contexte d'accroissement des aléas, les villes augmentent leur résilience en impliquant tous les acteurs et en organisant la solidarité à tous les niveaux en situation de crise.

Dans le même temps, la production alimentaire sur les terres littorales se réorganise. Malgré une disparition significative des surfaces agricoles, les décideurs recherchent, à partir de 2050, à sécuriser l'approvisionnement alimentaire, grâce, notamment, à la diversification des cultures et à la recherche de synergies entre systèmes aquacoles et agricoles. L'appropriation, bien que tardive, des enjeux de la hausse du niveau de la mer par les gouvernants et la société conduit, à partir de 2060, à la multiplication des formes de valorisation économique des littoraux (cités «lacustres », usines flottantes...) afin de disposer de temps pour des replis plus durables. La protection des écosystèmes littoraux permet l'adaptation in situ des écosystèmes, bien que les services écosystémiques soient souvent altérés, notamment lors d'événements extrêmes dont l'intensité s'est accrue. 


\section{Schéma}

de positionnement

des scénarios

LeS TRAJECTOIRES DES HUIT SCÉnARIOS ont été synthétisées dans un graphe (cf. Fig. 1) où sont croisés l'atténuation du changement climatique à l'échelle globale et les efforts d'adaptation des littoraux à la hausse du niveau de la mer. L'origine du repère représente la situation en 2020 , et l'extrémité de la flèche la situation en 2100. La trajectoire, de l'origine à l'extrémité de la flèche, décrit la proportion des efforts consentis pour l'atténuation du changement climatique (et donc principalement la réduction des gaz à effet de serre) et les efforts d'adaptation du littoral dans le monde, entre ces deux repères temporels. Des points d'inflexion dans les trajectoires sont précisés avec une année qui représente le début d'une décennie de changement marqué. Plus on monte sur l'axe des ordonnées, plus l'atténuation globale est importante dans le scénario considéré. Plus on se déplace vers la droite sur l'axe des abscisses, plus les efforts d'adaptation des littoraux sont prioritaires. Des efforts « négatifs » ont été envisagés, il s’agit de situations où les actions pour l'adaptation sont plus faibles que celles actuellement mises en œuvre, conduisant ainsi à une inadaptation croissante des littoraux. De même, des atténuations " négatives » ont été envisagées, qui correspondent à des situations d'accroissement des émissions de gaz à effet de serre.

L'état physique du contexte global en 2100 est repérable par un code couleur porté par le nom du scénario, au bout de chaque flèche de trajectoire : vert clair pour "modéré ", vert foncé pour « sérieux ", orangé pour " grave » et rouge pour " extrême ".

Pour chacune des trois familles définies précédemment (cf. 1.2), il s'agit de faire ressortir la trajectoire générale de la famille, ainsi que les stratégies mises en œuvre dans chaque scénario par les principaux acteurs, notamment en matière d'adaptation des zones littorales face aux projections d'évolution du niveau de la mer. 
Figure 1. Positionnement des 8 scénarios et des 4 états physiques globaux en 2100 selon les évolutions des efforts d'adaptation littorale et ceux d'atténuation du changement climatique

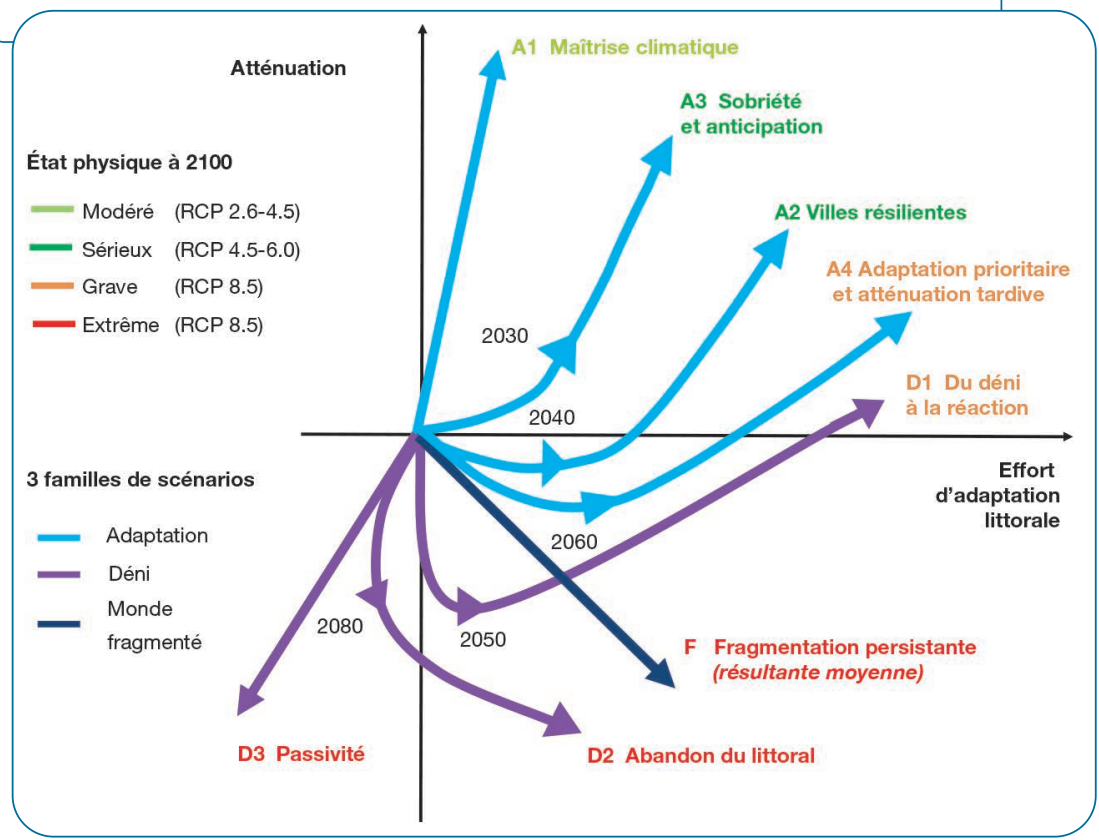

\section{Famille du " Déni "}

Cette famille Regroupe trois scénarios allant du déni durable à des situations de « réveils » plus ou moins tardifs. Le point commun de ces différentes variantes est l'attitude consistant, durant au moins la première moitié du xxl ${ }^{e}$ siècle, à refuser d'agir face aux risques pourtant de plus en plus incontestables liés à l'accélération du changement climatique et, en particulier, à la montée du niveau de la mer. Durant cette période d'immobilisme, les initiatives de prévention, tant en termes climatiques que d'adaptation littorale, ne constituent pas des priorités pour la communauté internationale et seuls quelques pays, très exposés et dotés de moyens financiers et technologiques suffisants, comme les Pays-Bas, prennent des initiatives isolées. Progressivement, toutefois, sous l'effet principal de la fréquence croissante d'événements climatiques catastrophiques en lien avec la montée des eaux, une prise de conscience de la criticité de la situation finit par émerger et contribuer à infléchir les politiques dans deux scénarios sur trois. Cependant, les situations résultantes en 2100 diffèrent sensiblement en termes de conséquences sur l’intégrité des littoraux. 
La trajectoire du scénario $\mathrm{D}$ 1, «Du déni à la réaction », commence par un déni à la fois du changement climatique (avec une augmentation des émissions de GES) et de ses effets sur les littoraux, puis bascule, après 2050, vers des actions massives de réduction des émissions de gaz à effet de serre et d'adaptation des littoraux, au prix d'efforts considérables. Si, après 2050 , les bénéfices des actions d'adaptation littorale se font rapidement ressentir (diminution de la vulnérabilité humaine et environnementale des littoraux), les premiers effets réellement perceptibles d'atténuation du changement climatique ne sont pas attendus avant le milieu du siècle suivant. Les mesures d'adaptation sont alors calibrées pour faire déjà face à une importante montée du niveau de la mer en 2100 et au-delà. La trajectoire du scénario D2, "Abandon du littoral », s’inscrit dans un déni du changement climatique et de ses impacts sur la hausse de la mer, puis après 2080 se caractérise par une transformation radicale des littoraux, sous la forme d'un retrait généralisé, le tout dans un contexte extrême de hausse du niveau de la mer (+ 1 à 2 m). Après 2080, peu d'actions d'atténuation des émissions de gaz à effet de serre sont engagées car on estime qu'il est désormais trop tard pour enrayer le changement climatique et ses conséquences. À l'échelle internationale, des investissements massifs sont consacrés à l'aménagement des littoraux et à l'organisation d'un repli stratégique sur l'intérieur des terres. Ces investissements visent à limiter les désastres humains mais surtout à prévenir les migrations massives susceptibles de mettre en péril un équilibre géopolitique déjà précaire. Les stratégies d'adaptation peinent à tempérer la forte exposition des littoraux aux risques de submersion et d'inondation, ce qui justifie leur fréquent abandon.

La trajectoire du scénario D3, "Passivité », se caractérise par un déni persistant du changement climatique et une passivité assumée en matière de gestion littorale dans un contexte extrême de hausse du niveau de la mer ( $2 \mathrm{~m}$; toujours en accélération). Malgré la multiplication des signaux d'alerte et en raison de l'absence de moyens communs affectés aux efforts de prévention et d'adaptation à l'échelle mondiale, l'attitude de déni continu aboutit à une politique court-termiste de « chacun pour soi ». En l'absence de politiques d'atténuation, une forte accélération de la hausse du niveau de la mer après 2050 provoque des transformations majeures des littoraux à l'échelle globale. Faute d'anticiper les effets de cette hausse, la plupart des zones littorales connaissent des crises humanitaires, alimentaires et environnementales répétées, à l'occasion d'épisodes de submersion marine et/ou d'inondation. Cela conduit à une dégradation accélérée des écosystèmes, une intensification des flux migratoires entre zones à risque élevé de submersion et zones de sécurité de long terme (mégapoles côtières, arrière-pays, pays, régions mondiales), et une brutalisation des rapports sociaux et géopolitiques.

\section{Famille " Fragmentation persistante "}

Cette famille aTyPique est constituée d'un Seul scénario qui rassemble des situations hétérogènes. Il s'agit d'une trajectoire « hybride » qui regroupe des situations locales aux 
caractéristiques parfois opposées, certaines représentatives de la famille des scénarios du déni et d'autres de celle des scénarios de l'adaptation. La cohabitation de caractères aussi contradictoires dans une seule et même trajectoire s'explique par le récit qui y est associé, celui d'un monde dual qui avance schématiquement à deux vitesses, selon la logique globale du « chacun pour soi ».

La trajectoire du scénario $\mathrm{F}$ « Fragmentation persistante » se caractérise par des efforts désordonnés d'atténuation globale du changement climatique du fait d'un nombre minoritaire de pays et des efforts d'adaptation très variables d'une région du monde à l'autre. L'absence de mobilisation sur la politique climatique globale aboutit à des émissions importantes de gaz à effet de serre et un contexte physique extrême d'élévation du niveau de la mer (+ $2 \mathrm{~m}$ ). Face à cela, on observe des situations aux caractéristiques opposées : les littoraux des pays riches font l'objet de travaux massifs et coûteux d'adaptation, alors que les littoraux des pays pauvres voient leur vulnérabilité s'accroître. Ce scénario de fragmentation reste sombre à l'échelle mondiale. Si certains États réussissent à « limiter la casse » sur leurs littoraux durant le siècle à venir (toutefois, à quel prix ?), la question qui se pose sur la pertinence des investissements colossaux consentis pour y parvenir est : « jusqu'à quand »?

\section{Famille " Adaptation littorale "}

Cette famille Regroupe quATRe SCÉnARIos constituant un corpus globalement vertueux même si les capacités d'anticipation, les efforts de prévention et l'intensité des conséquences peuvent différer. La famille se décompose en deux paires de trajectoires. La première, qui regroupe les trajectoires $A_{1}$ et $A_{3}$, correspond à des scénarios résolument proactifs, dans lesquels une prise de conscience rapide de la gravité de la situation, associée à la mise en œuvre de mesures efficaces d'atténuation, limite considérablement la gravité des conséquences (contexte physique global : " modéré » à " sérieux »). La seconde, qui regroupe les trajectoires $\mathrm{A} 2$ et $\mathrm{A} 4$, correspond à une évolution « à rebond », la prise de conscience et la mobilisation des politiques publiques pour l'atténuation intervenant plus tardivement dans le courant du siècle. Ce retard au démarrage dû à une attitude réactive a des conséquences plus préoccupantes que celles de l'attitude proactive car le contexte global final est soit « sérieux », soit " grave ».

La trajectoire du scénario A1, « Maîtrise climatique », conjugue de fortes politiques d'atténuation du changement climatique mises en place très précocement et des efforts modérés d'adaptation des littoraux, car répondant à une faible hausse du niveau de la mer. En effet, la mise en œuvre dès 2020 de fortes mesures de réduction des émissions de gaz à effet de serre (décarbonation de l'économie, meilleure efficience énergétique, technologies de capture et stockage du carbone...) par les acteurs nationaux a permis de limiter l'élévation du niveau de la mer. Les mesures d'adaptation des littoraux sont 
proportionnelles au risque de hausse du niveau de la mer qui reste modéré tout au long de la période ( $50 \mathrm{~cm}$ en 2100 avec une vitesse faible d'évolution).

La trajectoire du scénario $A_{3}$, « Sobriété et Anticipation », conjugue une gouvernance littorale anticipant les conséquences du changement climatique sur les littoraux (la hausse du niveau de la mer est plus importante que dans le premier scénario) et un basculement précoce, aux alentours de 2030, vers une économie de la sobriété, entraînant une forte réduction des émissions.

La trajectoire du scénario A2, «Villes résilientes », voit les grandes villes, en particulier les mégapoles littorales devenues le cœur de l'économie mondiale, jouer un rôle crucial dans l'adaptation littorale à la hausse du niveau de la mer et dans l'atténuation du changement climatique. Très tôt, les grandes villes organisées en réseaux d'expérimentation et de recherche sur l'adaptation littorale renforcent leur résilience face à la hausse du niveau de la mer. Puis, après 2040, les grandes villes et les États se coordonnent à l'échelle mondiale pour mettre en œuvre des mesures de réduction des émissions, ce qui a pour effet de limiter la hausse du niveau de la mer dans la deuxième moitié du xxle siècle.

La trajectoire du scénario A4, "Adaptation prioritaire et atténuation tardive », combinent une adaptation très précoce des littoraux à la hausse du niveau de la mer à une quasi-absence de mesures de réduction des émissions de gaz à effet de serre, qui ne sont mises en œuvre que tardivement, après 2060. Si cette quatrième trajectoire suit initialement la deuxième trajectoire en termes d'adaptation littorale, l'accélération de la hausse du niveau de la mer après 2050 oblige à accroître constamment les efforts d'adaptation littorale pour faire face à une situation grave $(+1 \mathrm{~m})$.

Cette large gamme de scénarios, ceux-ci étant issus de combinaisons cohérentes, robustes et plausibles d'une centaine d'hypothèses, mène d'emblée à quatre remarques majeures : - Si l'on écarte les deux scénarios les plus improbables (A1, « Maîtrise climatique » et D3, «Passivité persistante »), la majorité des scénarios (4 sur 6) conduisent à des situations globales graves ou extrêmes en matière d'élévation du niveau de la mer et de ses conséquences à la fin de ce siècle.

- Dans la plupart des scénarios, les trajectoires s’infléchissent avec un changement de priorité : les efforts d'atténuation du changement climatique prennent le pas sur ceux consacrés à l'adaptation des littoraux à la montée du niveau de la mer et aux événements climatiques extrêmes, ce qui réduit le risque de connaître une situation globale extrême en 2100.

- Plus le changement de priorité est précoce (passage de l'adaptation à l'atténuation), plus la situation à 2100 a des chances de s'améliorer.

- La trajectoire actuelle dans le monde est proche du scénario de la fragmentation qui conduit à une situation extrême en 2100.

Ces constats sont globalement inquiétants. La majorité des climatologues estiment que l'humanité ne dispose que de quelques années pour amorcer un infléchissement nécessaire confirmé par l'analyse prospective et que plus ce changement de cap sera précoce, moins il sera cher, en termes de coûts d'adaptation comme de crises. 
Le chapitre suivant se concentre sur une région française et sur deux pays étrangers afin de montrer concrètement comment se pose cette question de la hausse du niveau de la mer dans des contextes géographiques, politiques, économiques et sociaux différents. 


\section{Partie II}

\section{Focus territoriaux}

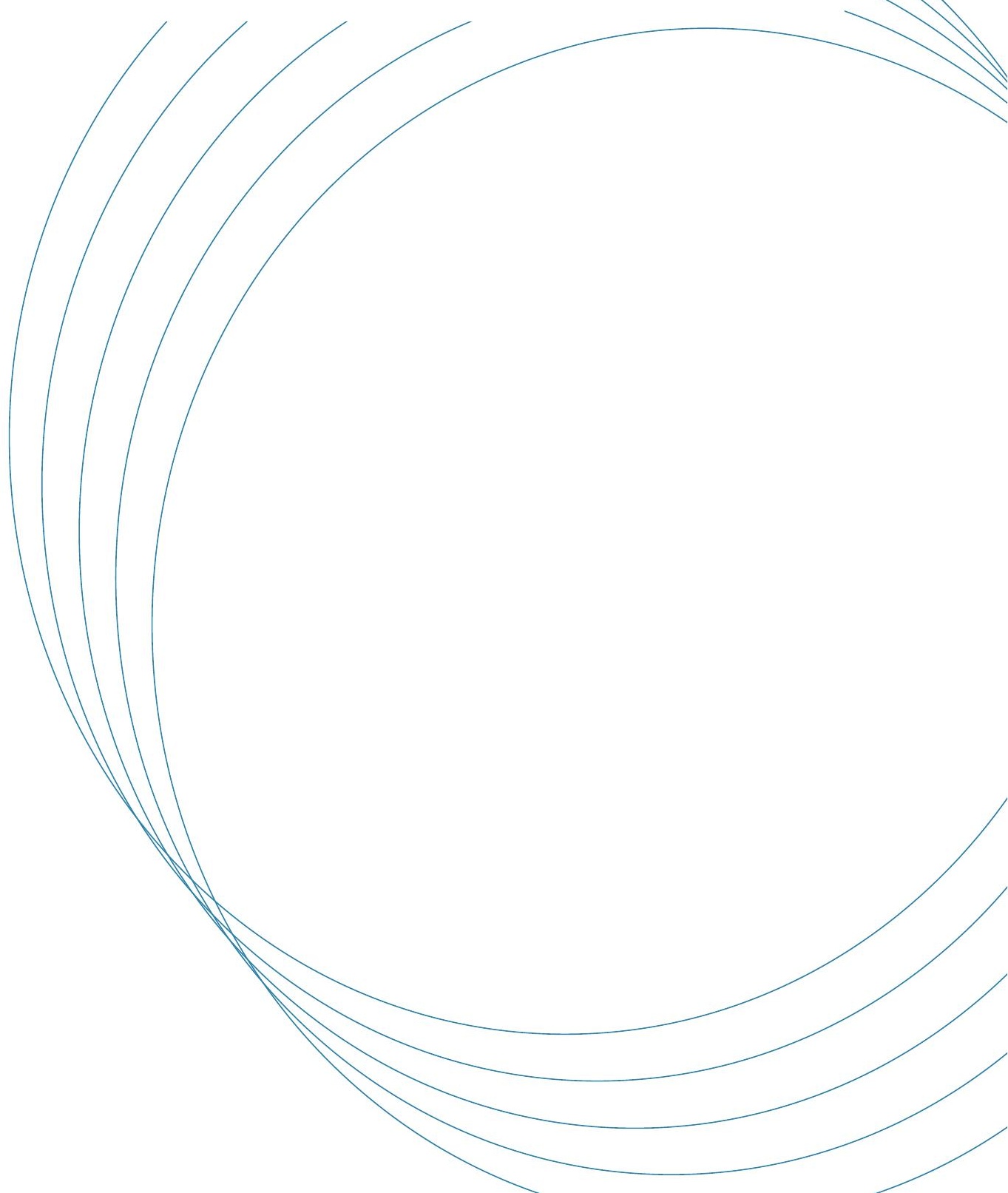




\section{Nouvelle-Aquitaine}

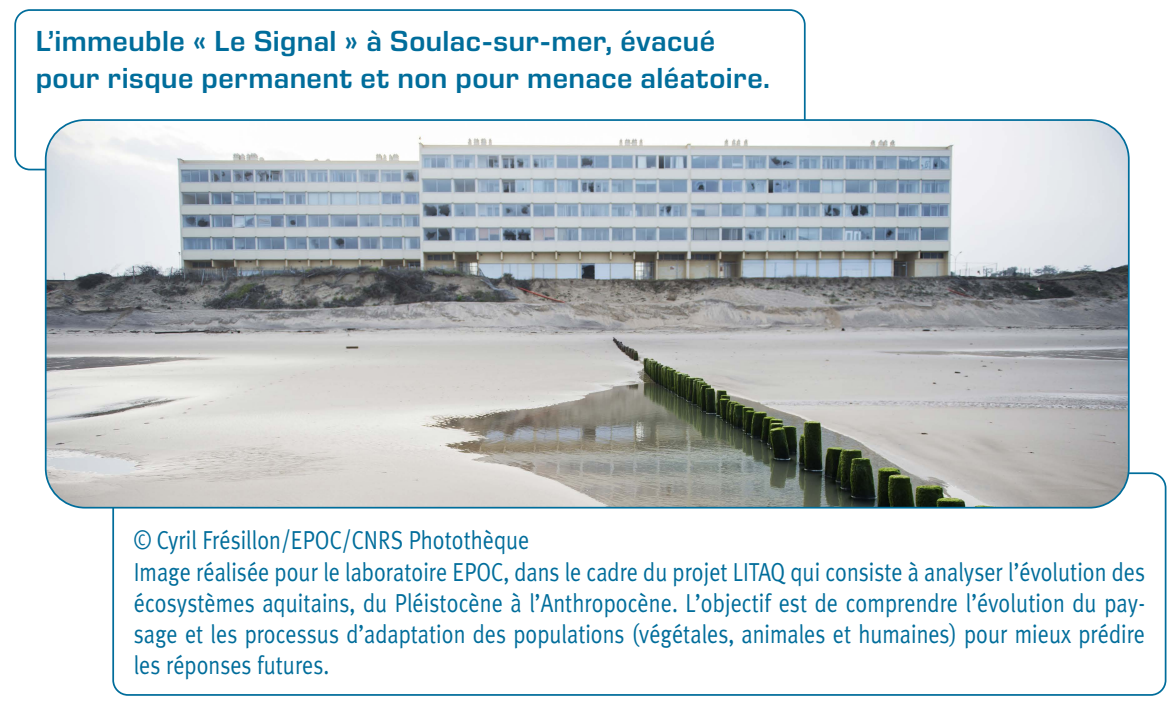

\section{Travaux d'enrochement sur la côte Aquitaine pour tenter de stabiliser} le cordon dunaire.

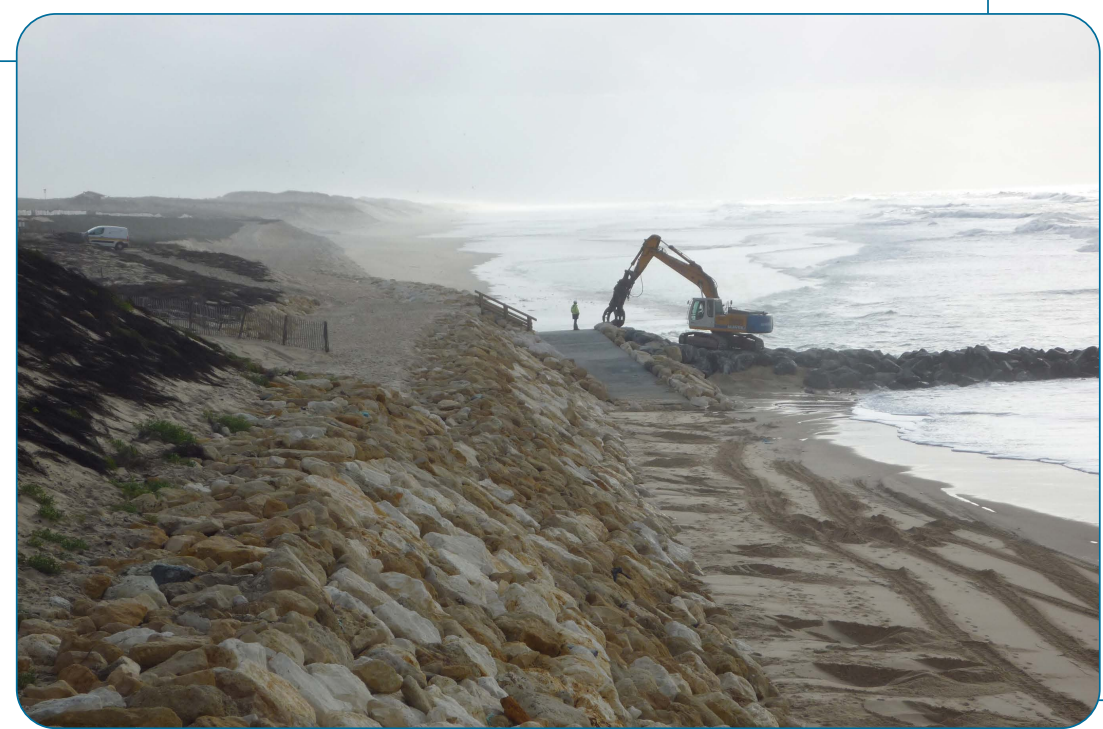

(c) Nicolas Rocle/INRAE 
Pays-Bas

Barrière amovible du chenal de Rotterdam Maeslant-Kettering, protégeant le port du risque de submersion marine.

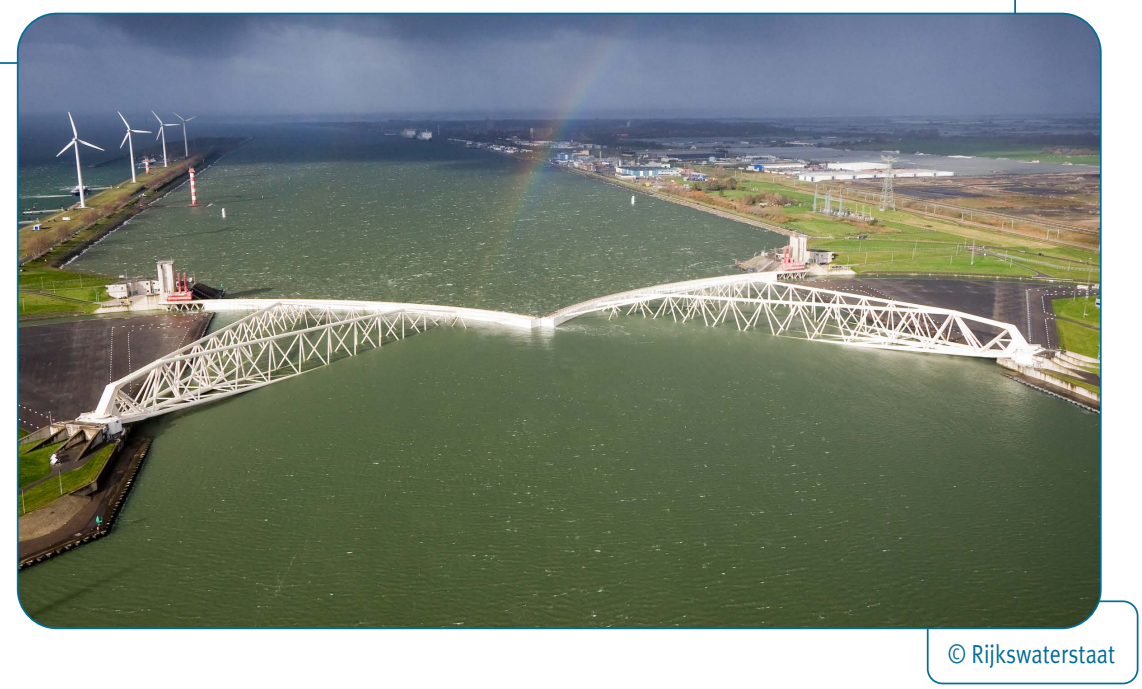

La rupture de digues en 1953 aux Pays-Bas entraîna une catastrophe majeure, justifiant la mie en place de l'ambitieux plan Delta sur 40 ans.

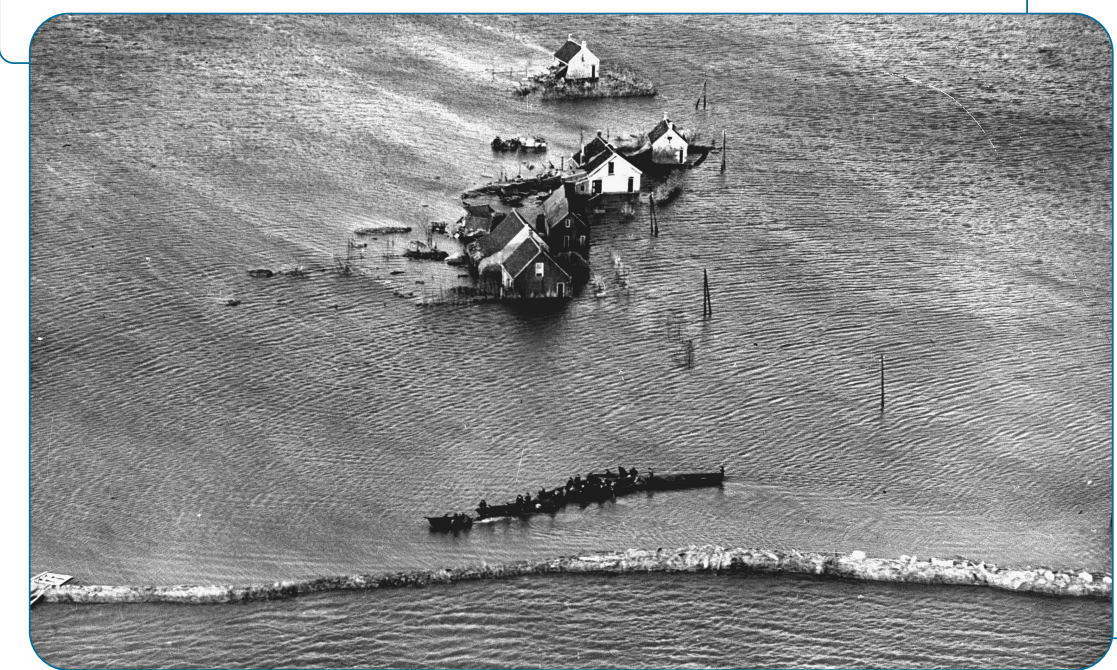

(C) Rijkswaterstaat 


\section{Vietnam}

Can Tho (1,2 million d'hab.), est la plus grande ville du delta du Mékong. Elle devient d'autant plus vulnérable à la submersion marine que les apports d'eau douce ont tendance à diminuer.

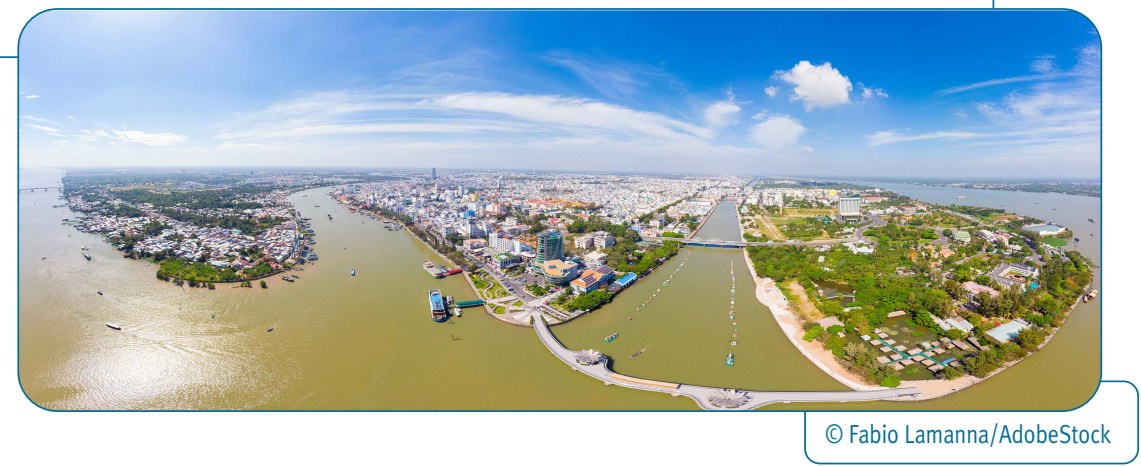

Recul de la côte dans la ville touristique de Cua-Dai-Hoi-An, au sud de Da Nang, province de Quang Nam.

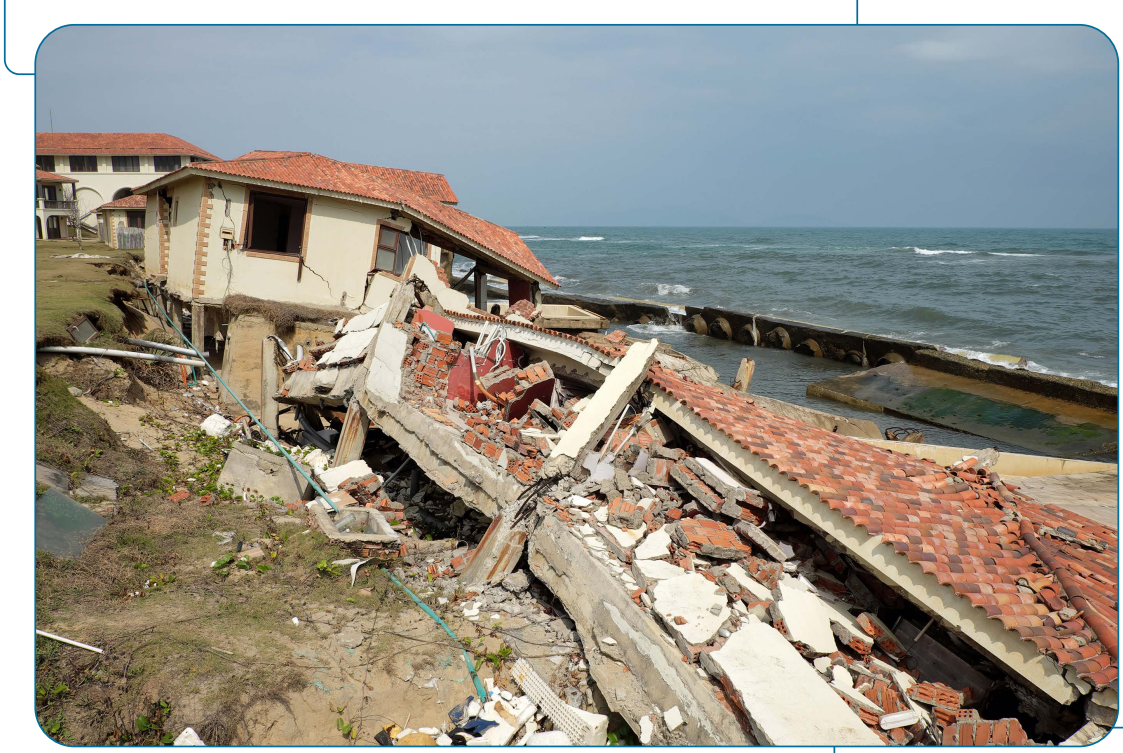

(c) Xuanhuongho/AdobeStock 


\section{La région Nouvelle- Aquitaine}

\section{Le Dittoral en Nouvelle-Aquitaine : diversifié, dynamique et attractif}

La RÉgion Nouvelle-Aquitaine est issue de la fusion en 2015 des trois anciennes régions Aquitaine, Limousin et Poitou-Charentes. Avec 12 départements totalisant $84000 \mathrm{~km}^{2}$, elle est la région la plus vaste de France. En dehors de la métropole bordelaise (900 ooo habitants) et des principales aires urbaines (Limoges, Poitiers, Pau, La Rochelle, Angoulême...), sa population globale, d'environ 5,9 millions d'habitants, se répartit principalement dans des villes moyennes ou des petites agglomérations. Le caractère rural de la région est notamment lié à l'importance de la forêt qui couvre environ le tiers du territoire et à une production agricole importante et diversifiée. Le dynamisme démographique, touristique et économique de la région est fort et tout semble indiquer que ces dynamiques vont se poursuivre : la richesse de ses patrimoines naturels et culturels, la démographie en constante progression (surtout sur le littoral), le développement de nombre d'industries régionales et des secteurs agricoles et touristiques en font un des territoires les plus attractifs de France.

En Nouvelle-Aquitaine, le linéaire côtier de 970 km (dont 4 îles) est très diversifié, autant en termes d'occupation et d'activités humaines qu'en termes de milieux et d'écosystèmes : côtes rocheuses, plages et barres sédimentaires, estuaires (dont celui de la Gironde, plus grand estuaire non endigué d'Europe), baies et estrans tidaux, ainsi que plaines côtières de faible altitude par rapport au niveau des plus hautes mers. Sur de larges portions de littoral naturel se sont développées une dizaine de stations balnéaires, avec des pôles ou des aires urbaines comme La Rochelle, Arcachon et la communauté d'agglomération du Pays basque. La fréquentation en hausse et la diversification des activités touristiques et récréatives caractérisent ce littoral riche et varié, mais les phénomènes d'érosion et de submersion marine, très hétérogènes dans le temps et dans l'espace, en font aussi un littoral vulnérable aux changements environnementaux.

\section{Dynamiques démographiques et économiques}

La bande côtière de Nouvelle-Aquitaine, structurée autour des villes et en premier lieu autour de la métropole bordelaise, concentre l'essentiel de la démographie avec $50 \%$ de la population résidant à moins de $60 \mathrm{~km}$ de l'océan. Le solde migratoire positif et une économie touristique importante expliquent l'augmentation continue des capacités 
d'accueil, dont $70 \%$ sont représentées par les résidences secondaires. L'emploi saisonnier est marqué, en raison de cette économie littorale et maritime, dont les principaux vecteurs d'identité sont la plaisance et les loisirs nautiques (La Rochelle est le $1^{\text {er }}$ port de plaisance de France), la pêche professionnelle et l'aquaculture (conchyliculture et mytiliculture), les industries navales et nautiques, les ports de commerce, ainsi que l'industrie de la glisse avec des entreprises de renommée internationale. La centrale nucléaire du Blayais, située sur la rive droite de l'estuaire de Gironde près de la commune de Blaye, a été mise en service en 1981 et produit environ 25 TWh par an. Le dynamisme économique du littoral néo-aquitain se caractérise par la diversité des formes productives, industrielles et de services, même si certaines activités primaires diminuent au profit d'activités tertiaires orientées notamment vers le tourisme et les loisirs de plein air.

\section{Dynamiques physiques et risques associés}

Suite aux tempêtes de l'hiver 2013-2014, une actualisation des projections de recul du trait de côte à l'horizon 2050 a été réalisée par l'Observatoire de la Côte NouvelleAquitaine (Bernon et al., 2016). Le recul moyen estimé pour le littoral aquitain à 2050 est ainsi de $50 \mathrm{~m}$ sur la côte sableuse et de $27 \mathrm{~m}$ sur la côte rocheuse. À ceci peuvent s'ajouter des reculs brutaux lors de tempêtes, de l'ordre de $25 \mathrm{~m}$ pour les deux types de côtes. La vitesse d'élévation du niveau de la mer est actuellement, au droit du littoral de la Nouvelle-Aquitaine, de l'ordre de $3 \mathrm{~mm} / \mathrm{an}$. Dans l'état actuel des connaissances, on sait que le climat affectera les zones littorales du fait de l'élévation du niveau de la mer et aussi du fait de la réduction des débits solides et liquides des bassins versants, notamment dans la Gironde. En revanche, les effets liés à d'autres facteurs de forçage sur la côte (intensité et fréquence des événements extrêmes, orientation de la houle et évolution des régimes de tempêtes) seront d'un ordre de grandeur inférieur. Parmi les conséquences de cet ensemble de phénomènes figurent, dans un premier temps, une aggravation perceptible des phénomènes de submersion marine, puis, ultérieurement, des transformations graduelles des paysages et des milieux littoraux et une accentuation des phénomènes d'érosion. Les effets sur les estuaires, les milieux limono-vaseux ainsi que les coûts et dommages associés demeurent aujourd'hui peu connus (Le Cozannet et al., 2016. Le Treut, 2018).

\section{Institutions et gouvernance du littoral}

Les dynamiques de politiques urbaines et d'aménagement du littoral néo-aquitain suivent des choix stratégiques faits depuis les années 2000. Ce cas d'étude fait en outre apparaître des différences notables entre le littoral de l'ex-Aquitaine et le littoral du département de Charente-Maritime, tant vis-à-vis des vulnérabilités liées au changement climatique que des logiques d'action en cours. 
Au plan historique, les principes d'aménagement posés par la Mission Interministérielle d'Aménagement de la Côte Aquitaine (MIACA) ont conduit à une urbanisation relativement concentrée sur les stations balnéaires et leur rétro-littoral, afin de maintenir de larges secteurs d'espaces naturels. L'observation et le suivi des dynamiques côtières, sur ce littoral très mobile depuis sa formation géologique, ont également conduit à de nombreux travaux de recherche et d'expertise, et à la création de l'Observatoire de la Côte Nouvelle-Aquitaine en 1996. Enfin, la volonté de pérenniser une action collective à l'échelle régionale s'est traduite par la création, en 2006, du Groupement d'Intérêt Public (GIP) « Littoral Aquitain », réunissant l'ensemble des collectivités territoriales du littoral de l'ex-Aquitaine et des services de l'État. Ce groupement est défini comme « un lieu de production d'études à caractère prospectif, mais aussi un outil de concertation, de mise en cohérence des projets, d'échanges d'expériences et de diffusion de bonnes pratiques entre ses membres et partenaires [dans] trois domaines principaux : l'organisation de l'espace littoral, la gestion de la bande côtière et l'aménagement touristique durable » (http://www.littoral-aquitain.fr/). Son périmètre d'action couvre désormais l'ensemble du littoral néo-aquitain.

À partir des travaux de l'Observatoire de la Côte Nouvelle-Aquitaine sur les dynamiques d'érosion et de recul du trait de côte, et dans le cadre du partenariat conduit par le Groupement d'Intérêt Public (GIP) Littoral Aquitain, une stratégie régionale de gestion de la bande côtière a été approuvée en 2012. Elle vise à encadrer et à accompagner les collectivités dans la mise en place de «stratégies locales de gestion de la bande côtière » sur les principales zones de vulnérabilité. Ces stratégies ne se substituent pas aux dispositifs réglementaires, tels que les plans de prévention des risques littoraux (PPRL), mais comportent diverses actions, depuis l'amélioration et la consolidation des connaissances, jusqu'à l'adoption de stratégies à moyen-long terme pour répondre aux tensions entre attractivité des stations, vulnérabilités liées à l'érosion, et protection des biens et des personnes (Rocle, 2017). Ainsi, le nécessaire abandon des constructions sur des dunes littorales menacées par l'érosion marine pose des problèmes juridiques et assurantiels complexes. C'est le cas emblématique de l'immeuble Le Signal, situé à Soulac-sur-Mer, dont les propriétaires ont fait l'objet d'une expropriation du fait des menaces d'effondrement prochain du bâtiment. La solution, encore à l'étude, serait le transfert de propriété de chaque lot à la Communauté de communes Médoc-Atlantique afin de pouvoir indemniser les propriétaires. De même, d'autres dispositifs prospectifs et d'ingénierie en partenariat avec les collectivités sont proposés, tels que la démarche "Aménagement durable des stations », et le futur schéma d'organisation de l'espace du littoral aquitain. Ces dispositifs visent ainsi à s'adapter aux dynamiques littorales et aux risques associés, tout en intégrant les nécessités de rénovation des stations balnéaires et de réflexion prospective en termes d'urbanisme, de mobilités et d'organisation de l'espace sur la frange terrestre du littoral, en incluant la préservation des espaces naturels, agricoles et forestiers. La figure 2 présente les stratégies d'aménagement et leur état d'avancement en 2021 : 
Figure 2. Cartographie de l'état d'avancement, en juin 2021, des stratégies locales de gestion de la bande côtière sur le littoral aquitain.

\section{STRATÉGIES LOCALES DE GESTION DE LA BANDE CÔTIÈRE}

\section{ÉTAT D'AVANCEMENT EN 2021}

île d'Oléron

Réflexion pour lancement

En phase d'étude

En phase opérationnelle

Opérationnelles engagées dans une actualisation

$$
\begin{array}{r}
\text { Agglomération } \\
\text { de Royan }
\end{array}
$$

Pointe Médoc Nord

Pointe Médoc Sud
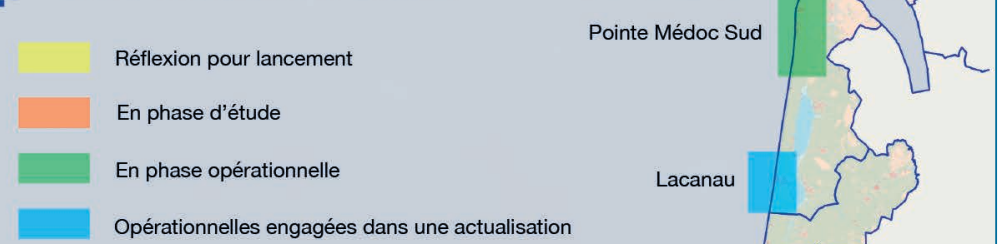

ge-Cap Ferret

La Teste-de-Buch

Biscarrosse

Lacanau

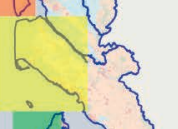

(

Côte Basque

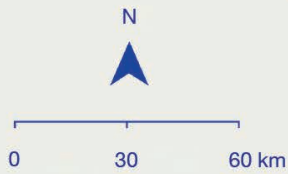

RÉALISATION : GIP LITTORAL, JUIN 2021

FOND DE CARTE : DONNÉES OCCUPATION DU SOL, GIP LITTORAL, 2015 


\section{Trois scénarios plausibles}

LES TROIS SCÉNARIOS SUIVANTS partent d'une même évolution climatique mondiale « de référence ", caractérisée par une élévation moyenne du niveau de la mer de $30 \mathrm{~cm}$ en 2050 et de $100 \mathrm{~cm}$ en 2100, avec une vitesse d'évolution toujours positive à la fin du siècle et des événements climatiques extrêmes plus fréquents et plus forts.

\section{Scénario 1 : Des stratégies de gestion à la dérive}

La croissance démographique n'ayant pu être freinée sur le littoral de Nouvelle-Aquitaine avant 2050, la plupart des stations balnéaires ont accueilli de nouveaux résidents et ont fait l'objet d'une rénovation importante (logements, infrastructures, cadre de vie). Cette attractivité a incité nombre d'élus locaux à décider (avec l'aide de diverses expertises) le renfort des mesures de protection, parfois avec des ouvrages massifs dont les coûts associés ont endetté les collectivités sur plusieurs décennies.

Cependant, malgré le foisonnement d'initiatives locales pour contrecarrer les effets de l'érosion et des submersions (de plus en plus violentes à chaque tempête), les collectivités peinent à s'adapter au rythme des dégâts et des dommages sur le littoral. Ceci est encore exacerbé en Charente-Maritime, où les submersions couvrent à chaque fois de plus grandes étendues de marais, mais aussi d'habitations. Le pôle de La Rochelle est particulièrement déstabilisé, notamment au niveau des ports de plaisance et des aménagements en front de mer.

Les stratégies locales de gestion de la bande côtière, initiées dans les années 2010, n'ont donc pas entraîné de changements dans la manière de penser et de vivre sur le littoral, tout juste ont-elles permis de renforcer les connaissances sur les phénomènes et de limiter les risques de non-conformité aux exigences européennes parues dans les années 2030. Les intercommunalités, qui ont repris toutes les compétences et prérogatives en matière de "défense contre la mer », ont du mal à s'entendre sur les actions à mener car tout ouvrage de protection à la côte a des effets en aval de la dérive sédimentaire.

Un sursaut a cependant eu lieu en $\mathbf{2 0 4 5}$, alors que le recul du trait de côte se faisait de plus en plus important à chaque hiver, et que les dégâts des tempêtes commençaient à faire vaciller le système d'indemnisation des catastrophes naturelles mis en place en France en 1982. L'État a alors entrepris une révision du système assurantiel, conditionnant en particulier la reconnaissance de l'état de catastrophe naturelle à de nouvelles mesures de prévention et de gestion de crise. De même, l'aggravation de l'érosion côtière a donné lieu à la création d'un fonds d'aide pour la recomposition littorale, permettant des acquisitions et des indemnisations par anticipation et la prévention de risques pour les personnes et les biens. Mais cet ajustement n'était déjà plus à la hauteur de l'anticipation que réclamaient des scientifiques, des organisations non gouvernementales et des mouvements citoyens à la suite du Sommet d'Abidjan en 2035.

Les conséquences de cette course contre les événements météo marins deviennent alors de plus en plus lourdes pour la région. Parmi les territoires les plus touchés par la 
montée du niveau de la mer, le bassin d’Arcachon et ses infrastructures périphériques (ports, routes, habitations, réseaux d'eau et d'assainissement...) sont progressivement fragilisés. Des phénomènes de pollution des eaux de plus en plus fréquents mettent à mal les filières conchylicoles, ainsi que la fréquentation touristique en raison des interdictions de baignade et des épisodes de submersion répétés.

C'est donc à un abandon progressif, mal coordonné et forcé, que doivent se résoudre les élus locaux et les populations concernées dans la seconde moitié du xxle siècle. Les ruines accumulées au pied des falaises rocheuses et la presqu'île disparue du Cap Ferret constituent, en 2050, les marqueurs d'une modification profonde des paysages tant recherchés par les touristes et les amateurs de sentiers littoraux au début du siècle. Cet « exode littoral » entraîne une augmentation rapide de la population dans l'aire métropolitaine bordelaise, qui révise alors ses schémas d'aménagement tous les 3 à 5 ans, aussi bien pour intégrer cette urbanisation galopante que vis-à-vis des soubresauts de l'estuaire dont on ne sait plus très bien appréhender la dynamique d'évolution en 2100.

\section{Scénario 2 : Aquitania ou la dépoldérisation du bassin aquitain}

«Vivre avec le risque » : ce mot d’ordre est apparu dans les années 2010 alors que les gouvernements européens voyaient poindre de sérieux problèmes quant aux effets du changement climatique sur les littoraux. Il est devenu le paradigme de la gestion des risques dans les années 2020, et a été particulièrement moteur en Nouvelle-Aquitaine, parallèlement aux efforts importants d'atténuation des émissions de gaz à effet de serre des pays européens.

Ainsi, dès 2022, avec les nouveaux « fonds européens de développement régional et de résilience territoriale ", la région et l'État ont-ils affirmé plus nettement encore que les aménagements et les modes d'occupation du littoral devaient être rendus compatibles avec un accompagnement des processus naturels, une libre évolution des cours d'eau et des estuaires, ainsi que les dépoldérisations provoquées par les tempêtes et l'élévation du niveau de la mer. Ces sources de financement étant devenues indispensables pour les collectivités, ces dernières ont tout mis en œuvre pour satisfaire à ces nouvelles exigences, devenues bientôt de véritables principes directeurs pour nombre d'acteurs du littoral.

Des collaborations avec les Pays-Bas et certains pays d'Asie du Sud-Est ont notamment permis, par le biais de réseaux scientifiques, d'associations, de collectifs d'architectes-urbanistes et d'aménageurs, de développer des formes innovantes d'habitats modulables, réversibles et pour certains flottants, afin de les déployer dans les zones rétro-littorales. Cela n'a pu être possible qu'à partir du moment où de nouvelles modalités de gestion souple du trait de côte ont été mises en œuvre, en écho à certains programmes d'expérimentation et de démonstration portés par le Conservatoire du littoral, l'Office Française pour la Biodiversité (OFB), des syndicats mixtes... Le concept de « solutions fondées sur la nature » a fait florès, d'abord porté par quelques associations environnementales, puis rapidement réapproprié dans de nombreux secteurs d'activité : nouvelles formes 
d'élevage dans les marais et plaines côtières, aquaculture durable, agriculture diversifiée et vivrière, filière bois-énergie, valorisation des espaces ennoyés par de nouvelles activités récréatives et sportives (sports de glisse, tourisme aquatique, etc.).

La tempête de 2047 a alors eu un tout autre retentissement que ce que les villes littorales avaient eu l'habitude de vivre : on ne déplorait plus les dégâts à la côte, on observait plutôt les effets sur la morphologie littorale afin de s'en inspirer et d'innover toujours plus dans les formes d'occupation et de valorisation plus éphémères, sensibles et artistiques que patrimoniales. Une bonne partie des métiers du littoral ont ainsi été créés à partir de ces nouvelles relations aux lieux et aux paysages, le littoral n'étant plus le lieu de villégiature de naguère, mais un gisement de ressources alimentaires, économiques, symboliques...

À partir des décennies 2060-2070, l'élévation du niveau de la mer a induit une profonde modification du linéaire côtier, des dunes, des estrans, des marais et des landes. La Charente maritime est désormais soumise en partie au va-et-vient des marées, ce qui induit de nouvelles activités de pêche et d'aquaculture, notamment la culture d'algues pour la santé et les cosmétiques. La vigne n'est plus cultivée sur la rive gauche de l'estuaire de la Gironde, et le démantèlement de la centrale du Blayais a été anticipé par la mise en place de turbines hydroélectriques et d'éoliennes. Lacs, marais, étangs et autres milieux humides composent désormais une mosaïque bleue, verte et saumâtre à l'échelle du bassin aquitain.

À la fin du siècle, le visage de la Nouvelle-Aquitaine est totalement différent de ce qu'il était 100 ans auparavant : les métiers, les filières, les pratiques de loisirs s'insèrent en majorité dans une économie bleue où l'eau est au cœur des efforts de préservation et de développement soutenable. L'élévation du niveau de la mer, qui se profile encore pour plusieurs siècles, est ainsi accompagnée, appropriée et mobilisée comme vecteur déterminant du devenir de la région.

\section{IScénario 3 : Les métropoles et leurs presqu'îles}

Le renforcement des politiques de prévention et de précaution face aux risques littoraux, en France et en Europe, a progressivement conduit entre 2020 et 2050 à freiner les mobilités démographiques vers les zones côtières, au profit d'une densification des arrière-pays mais aussi d'un petit nombre de métropoles proches de la mer. En NouvelleAquitaine, les politiques d'aménagement ont ainsi cherché à réduire l'urbanisation sur la bande littorale, tandis que la métropolisation sur les pôles bordelais et rochelais s'est poursuivie selon un rythme soutenu.

Parallèlement, les mesures de protection se sont renforcées là où les enjeux socioéconomiques étaient les plus forts, c'est-à-dire sur la plupart des stations balnéaires, notamment au Pays basque et dans l'aire de La Rochelle. Le bassin d'Arcachon a dû être réaménagé en profondeur (protection, dépoldérisation et relocalisation selon les sites à enjeux) afin d'atténuer au mieux les effets de l'élévation du niveau marin. Les coûts 
élevés de ces travaux ont été supportés par de nouvelles offres de biens et de services à destination des résidents et des touristes.

Les stations littorales, devenues des antennes-relais des métropoles grossissantes, évoluent alors sur le plan de leur aménagement comme de leur fonctionnement : la protection contre les aléas climatiques est renforcée, les réseaux de transport intelligent se sont développés pour accompagner des mobilités quotidiennes et des excursions depuis les métropoles, selon un nouveau modèle économique où les stations balnéaires doivent constamment s'adapter à une demande labile en termes de fréquentation touristique et récréative.

Dans les zones à plus faibles enjeux, vastes espaces entre métropoles régionales et villes à forte valeur patrimoniale ou touristique et les infrastructures de transport surélevées qui les relient, des formes de gestion souple sont appliquées afin d'accompagner les processus naturels à l'œuvre dans ces nouveaux « territoires du vide ». Les marais et les dunes sont ainsi laissés en libre évolution, ce qui conduit à un fort recul de la côte à partir de 2050, notamment en Charente.

Ces deux mécanismes (protection des stations balnéaires, et « laisser-faire » ailleurs) composent progressivement un linéaire côtier disparate, marquant une alternance forte entre les secteurs urbanisés et les anciens secteurs d'espace naturel. Un littoral « en pointillé » se dessine au fil des décennies avec une suite de « presqu'îles » sur la côte sableuse de la Charente aux Landes. Le Pays basque investit massivement dans la protection des côtes rocheuses, mais le niveau de la mer et les modifications de courants et de houle transforment la morphologie des plages et des barres sous-marines : des conflits avec les surfeurs, les pêcheurs et autres « gens de mer » deviennent chroniques. Vers la fin du siècle, la divergence entre les principales aires urbaines et les zones en libre évolution est de plus en plus marquée. Ces transformations aboutissent à un maillage dense de réseaux de transport et d'infrastructures entre les métropoles et les presqu'îles du littoral aquitain, ce qui renforce les interdépendances économiques et écologiques entre ces pôles. Ces évolutions s'accompagnent d'une diminution des surfaces agricoles et forestières dans l'arrière-pays, ce dernier étant désormais soumis à un mitage et une artificialisation difficilement contrôlée, compromettant les capacités de résilience et d'adaptation des espaces naturels, agricoles et forestiers d'une partie de la région. 


\section{Les Pays-Bas}

\section{Un pays de terres basses et d'eau}

Les Pays-Bas couvrent 41526 KMㄹ $^{2}$ dont 1/4 des terres est située sous le niveau de la mer et $1 / 6^{e}$ est constitué d'eau. Cette démocratie parlementaire ancienne compte 17,2 millions d'habitants (2019) et est l'un des membres fondateurs de l'ONU, de l'UE, de l'OTAN, de l'OCDE et de l'OMC. Pays riche, considéré comme très avancé en technologies d'aménagement hydraulique, les Pays-Bas figurent dans le haut du classement de l'indice du développement humain (IDH).

Les Pays-Bas sont traversés par $600 \mathrm{~km}$ de rivières et accueillent le delta de trois fleuves (Rhin, Meuse, Escaut) qui se jettent dans la mer du Nord. Les zones inondables représentent $60 \%$ de la superficie du pays, soit une menace pour 9 millions d'habitants, dont les grandes villes d'Utrecht, Amsterdam, La Haye et Rotterdam. Dans ce contexte hydrologique, les Pays-Bas se sont dotés de $3700 \mathrm{~km}$ de défense primaire contre les inondations et de plus de $14000 \mathrm{~km}$ de réseau secondaire. Il s'agit principalement de digues, traditionnellement construites en argile, et d'un renforcement des dunes naturelles. La partie centrale du pays est particulièrement vulnérable aux risques d'inondations et de submersion marine, avec un point bas à presque $7 \mathrm{~m}$ au-dessous du niveau de la mer, ce qui nécessite un pompage permanent en période de crue.

\section{Gouvernance et gestion de l'eau}

Il existe aux Pays-Bas une véritable gouvernance de l'eau, qui remonte au Moyen Âge. À la fin du x॥le siècle ont commencé à se constituer des autorités régionales de l'eau ou Waterschappen. Ces conseils de l'eau avaient trois missions, coordonnées par un maître de digue : la gestion des digues, celle des systèmes de drainage, l'arbitrage des conflits. Pour financer les travaux de construction et d'entretien, les Waterschappen ont introduit une taxe imposable à tous les citoyens, taxe basée sur la valeur réelle de leurs biens à protéger, quel que soit son rang ou sa profession. Cette taxe a été étendue depuis 2009 sur l'ensemble du pays, même dans les zones non vulnérables. À cet égard, les Waterschappen sont considérés comme l'un des premiers dispositifs territoriaux élaborés pour la gestion collective de l'espace (Gueben-Venière, 2015).

On peut ainsi parler d'une « démocratie fonctionnelle » pour la gestion de l'eau. Celle-ci s'appuie aujourd'hui sur les autorités régionales de l'eau dont les membres sont élus directement par les résidents et des représentants des usagers, et qui dispose d'une fiscalité propre. Dans cette gestion sont également impliqués les différents pouvoirs territoriaux, les entreprises et les instituts de recherche. Chaque maison, bâtiment ou terrain 
reçoit une estimation annuelle de sa valeur par la mairie, en suivant les prix de marché, ce qui facilite la collection des impôts (nationaux, locaux et régionaux) et les relocalisations.

Les enjeux de la gestion de l'eau, variables selon les lieux, sont de trois ordres :

- le risque de submersion marine (sur les côtes, avec un impact de la montée de la mer) ;

- le risque d'inondation par les fleuves et rivières (dans l'intérieur du pays) ;

- le risque de sécheresse en été (sur les hautes terres).

Les Pays-Bas consacrent 7 milliards d'euros par an pour la gestion de l'eau, principalement pour la prévention des inondations fluviales (90\% du budget) et la gestion de l'eau. Ce budget est alimenté par les taxes prélevées sur les propriétaires fonciers et les usagers de l'eau. La stratégie de gestion du risque lié à l'eau sur les zones littorales combine plusieurs actions complémentaires menées en parallèle :

- le renforcement des dunes par des apports de sable (en moyenne tous les 2 ans) ;

- la construction et l'entretien de digues sur les fleuves ;

- la barrière amovible (Maeslantbarrier) destinée à protéger la ville et le port de Rotterdam contre les tempêtes exceptionnelles et contre les surcotes (fermée deux fois depuis son inauguration en 1987);

- l'expérimentation d'un «Zandmotor» (ou « machine à sable ») où l'apport de 21 millions de $\mathrm{m}^{3}$ de sable extraits de la mer du Nord vise à renforcer l'approvisionnement du cordon littoral en utilisant le courant de dérive littorale comme moteur naturel de transport des sédiments.

\section{Le plan Delta}

À l'échelle nationale, la gouvernance de l'eau repose essentiellement sur le plan Delta. Celui-ci assure la coordination des différentes échelles (région, province, pays) afin de mobiliser tous les acteurs autour d'objectifs communs.

Le plan Delta a été élaboré par la commission éponyme suite à la tempête catastrophique de 1953, qui causa presque 2000 morts, provoqua l'évacuation de 70000 personnes, et entraina une perte de $5 \%$ du PIB (10 ooo têtes de bétail se noyèrent et 4500 bâtiments furent détruits). Ce plan consista à fermer tous les bras de mer, à l'exception de l'Escaut occidental et du Nieuwe Waterweg, par des barrages. Dix barrages furent construits en trente ans et les digues systématiquement rehaussées. Le projet fut achevé en 1996, soit 43 ans après la catastrophe. Une autre mesure de protection fut l'aménagement de grands lacs d'eau douce pour éviter les inondations, ces lacs étant par ailleurs utilisés pour l'agriculture. En réduisant le risque d'inondation et de submersion à un niveau acceptable par les habitants, « le plan Delta a largement conforté une vision techniciste de la gestion des eaux et du littoral, consistant à repousser la mer au-delà de digues et barrages et à continuer de conquérir de nouvelles terres pour ne plus jamais revivre un tel drame » (Gueben-Venière, 2015).

Considéré au départ comme un plan stratégique de protection, le plan Delta est maintenant adossé à un agenda d'investissements permanents qui se décline en trois volets : 
l'aménagement de l'espace (en particulier urbain), la gestion de l'eau, la gestion des catastrophes et des inondations. La gouvernance d'un système aussi complexe exige de bien coordonner les différents acteurs aux trois échelles pertinentes : locale, régionale et nationale.

Un changement de philosophie a vu le jour dans les révisions successives du plan Delta. En effet, celui-ci a d'abord été construit avec l'objectif de protéger les populations grâce à des solutions technologiques consistant à repousser la mer au-delà de digues et de barrages et à continuer de conquérir de nouvelles terres. Mais depuis quelques années, il a fait l'objet de contestations de la part des populations, sur le plan économique, environnemental et même politique. Il s'agit désormais de « vivre avec les aléas météo marins plutôt que de s'y opposer. [...] En effet, le challenge des Pays-Bas pour ce siècle ne concerne pas une menace à combattre. [...] La question fondamentale est plutôt : comment pouvons-nous nous assurer que notre pays sera toujours attractif, pour y travailler comme pour y investir et y vivre pour les générations futures ? (Delta Commissie, 2008).

L’idée directrice du plan Delta actuel est désormais de protéger en réduisant le risque d'inondation, mais aussi d'anticiper l'impact d'une inondation possible. Il s'agit donc de : - réduire la probabilité d'occurrence d'une inondation par des mesures appropriées comme des ouvrages de protection;

- réduire les dommages en cas d'inondation par l'aménagement de l'espace (aménagement urbain, architecture résiliente aux inondations). En effet, l'augmentation du niveau de protection ne résoudra pas tout. Par exemple, le programme "Room for the river» met en œuvre un déplacement d'habitations et de population pour créer une zone d'expansion temporaire des crues ;

- se préparer à gérer une catastrophe et anticiper les réponses (restauration des ouvrages, réhabilitation des zones affectées).

Les décideurs évoluent donc vers une gestion intégrée du territoire répondant simultanément à plusieurs enjeux : défensifs, environnementaux, économiques et récréatifs. Cette ambition implique la solidarité entre les différentes autorités régionales de l'eau pour le financement des investissements.

Les principes du plan Delta intègrent désormais cette philosophie :

- le risque individuel acceptable d'inondation est le même pour tous les habitants du pays ;

- toutes les instances de décision doivent être impliquées ;

- la stratégie privilégie la capacité d'adaptation : garder les choix ouverts, faire ce qui est nécessaire, combiner investissements de court terme et stratégies de long terme, travailler avec la nature (par exemple, le rechargement des plages) ;

- la garantie de la continuité de l'action repose sur l'engagement budgétaire institutionnel de l'État ; à cette fin, il est sanctuarisé un fond dédié « Delta » de 1 milliard d'euros par an. Un commissaire est responsable selon la loi (Delta act) d'un rapport annuel au parlement ;

- la surveillance et l'évaluation continue des stratégies intègre la détection des signaux faibles et des procédures de révision ; 
- Les projections sur le futur à l'horizon de 100 ans doivent tenir compte des contraintes suivantes : une hausse du niveau de la mer comprise entre 20 et $100 \mathrm{~cm}$, des tempêtes plus fortes, une érosion croissante de la côte, un accroissement du débit des fleuves de $10 \%$ en hiver, une diminution du débit de $80 \%$ en été, et donc plus de sécheresse estivale, une subsidence (tassement du sol) de $10 \mathrm{~cm}$ dans les zones de tourbe, un développement spatial et une artificialisation des sols, avec un million de maisons supplémentaires, une salinisation des terres.

\section{Une révision en cours du plan Delta}

Le plan Delta est en cours de révision. En effet, si ce plan présente une certaine robustesse à l'horizon 2050, de nouveaux éléments comme une accélération de la montée du niveau de la mer à l'horizon 2100, pourrait conduire à sa révision en profondeur. La figure 3 montre les hypothèses de hausse du niveau moyen de la mer envisagées pour les Pays-Bas en 2050, 2075 et 2100.

Figure 3. Hypothèses d'évolution du niveau moyen de la mer.
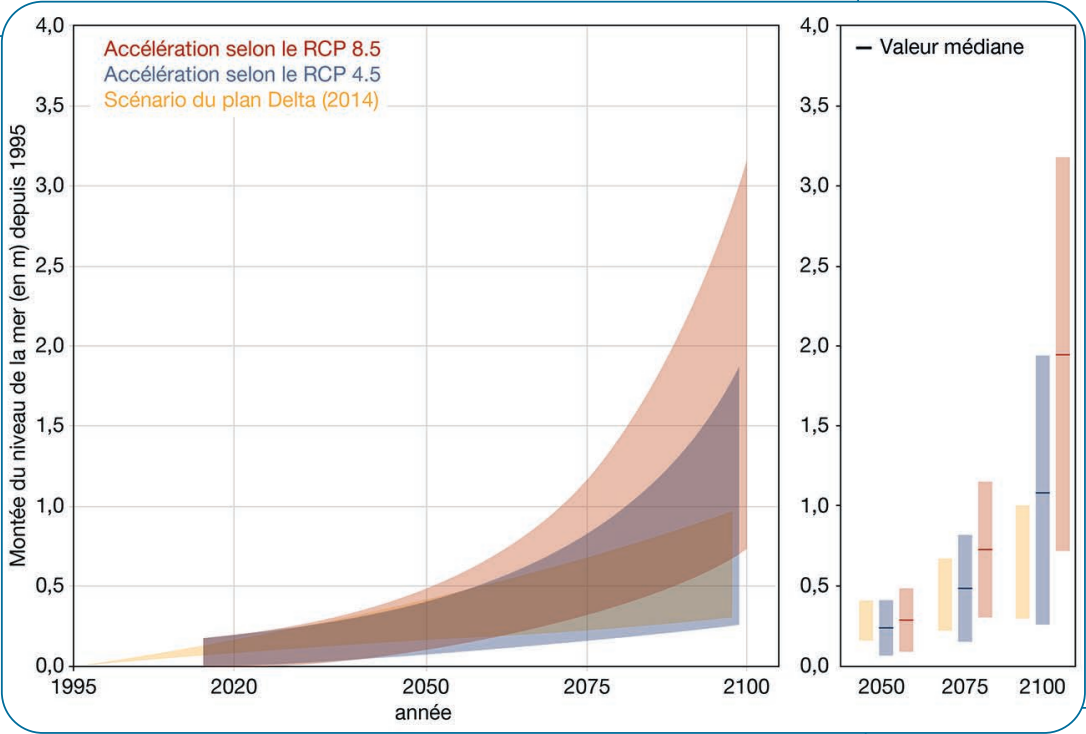

Haasnoot et al., 2020 
Actuellement les Pays-Bas font face à une élévation de $2 \mathrm{~mm} / \mathrm{an}$ le long de la côte, mais on constate déjà une accélération du phénomène (signal donné par le système de surveillance ainsi qu'un rapport en cours sur la fonte des glaciers de l'Antarctique). Un travail du Delta commissionner est à l'étude sur les conséquences d'une élévation des températures de $4^{\circ} \mathrm{C}$ en 2100. La hausse du niveau de la mer passerait alors de 1 à $2 \mathrm{~m}$, voire $3 \mathrm{~m}$ sur les côtes néerlandaises. Dans ce cas, la dynamique pourrait s'accélérer sur la période 2020-2100, et passer de $+15 \mathrm{~mm} / \mathrm{an}$ en début de période, à $+60 \mathrm{~mm} / \mathrm{an}$ en milieu de période, pour finir à $+100 \mathrm{~mm} / \mathrm{an}$ en fin de période. Cette accélération pose la question des capacités d'adaptation à long terme du territoire néerlandais.

Ainsi, dans le pire cas de figure, l'efficacité des plans pourrait aller décroissant (cf. figure 4) :

- un plan pour juguler une élévation du niveau de la mer de $50 \mathrm{~cm}$ aurait une durée de vie (efficacité) de 60 ans ;

- le plan suivant pour lutter contre une hausse de $1 \mathrm{~m}$ du niveau de la mer aurait une durée de vie de 20 ans ;

- le plan suivant pour lutter contre une hausse de 1,5 m du niveau de la mer aurait une durée de vie de 10 ans.

Ces projections doivent être rapprochées du fait que les travaux du premier plan ont pris 40 ans à être réalisés. De plus, une telle hausse entraînerait une très forte demande pour le rechargement des plages, opération dont le coût risquerait de croître jusqu'à devenir insoutenable. Enfin, la barrière initialement prévue pour protéger le premier port d'Europe, actuellement fermée tous les 10 ans, devrait être fermée environ 30 fois par an, dans ce cas de figure. Il y a des doutes sur la capacité de cet équipement à répondre à de telles sollicitations sans compter les perturbations du trafic portuaire. 
Figure 4. Délais potentiels de réaction face à une élévation de $50 \mathrm{~cm}$.

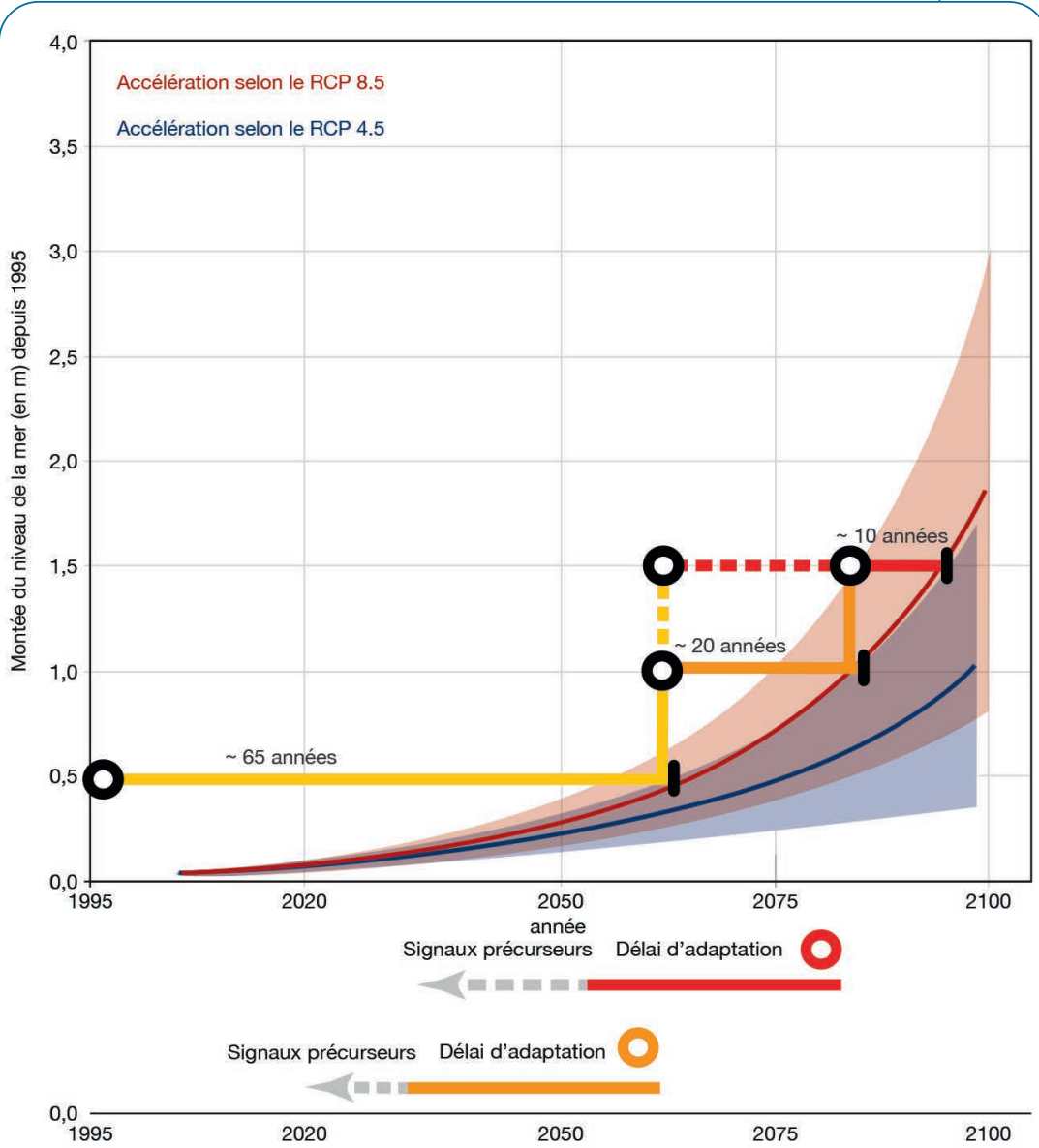

Les barres horizontales colorées représentent la durée de réalisation et de validité des aménagements faits pour une adaptation à une hausse de $0,50 \mathrm{~m}$. Si l'on suit la ligne rouge de la trajectoire moyenne de hausse du scénario du changement climatique le plus fort (RCP 8.5), le plus probable à ce jour, on observe que cette durée de validité décroît de 65 ans à 20 ans, puis à 10 ans pour chaque hausse de $0,50 \mathrm{~m}$. La ligne en pointillé montre que, si l'on anticipe dès 2060 une hausse de $1 \mathrm{~m}$ (tireté jaune), on dispose alors d'un délai d'adaptation de 30 ans (tireté rouge) pour une hausse cumulée de 1,50 m vers 2090. 


\section{Les scénarios possibles : quelles trajectoires d'adaptation pour le " Dutch Delta " à 2100 ?}

La trajectoire actuelle des Pays-Bas est celle de la conservation d'une forte capacité d'adaptation à la hausse du niveau de la mer et aux inondations, à la fois du point de vue de la gouvernance et des technologies déployées. Cependant peu d'actions sont engagées pour réduire les émissions de gaz à effet de serre et le modèle de développement des Pays-Bas reste très émetteur. De ce point de vue, on reste sur une trajectoire conventionnelle de développement. Mais une telle trajectoire d'adaptation peut-elle être durable à l'horizon 2100, notamment s'il y a une accélération du changement climatique et une accélération forte de l'élévation du niveau de la mer ? On peut imaginer deux scénarios contrastés selon les choix de gouvernance du pays sur plusieurs décennies.

\section{Scénario 1 : La technologie a ses limites}

Ce scénario considère une trajectoire conventionnelle de développement avec le maintien de la logique actuelle, basée sur la confiance générale, y compris politique, dans le « tout technologique ». Les Pays-Bas continuent à mettre en place une gestion adaptée à la montée de la mer en s'appuyant sur les technologies, la modélisation et l'intelligence artificielle, portée par une gouvernance de l'eau forte et intégrée. Ils cherchent à maintenir le trait de côte à tout prix, mais en l'absence d'actions d'atténuation significative à l'échelle mondiale, le changement climatique s'emballe et les techniques de rechargement des plages et de protection des zones sensibles sont progressivement mises en échec.

Après 2050, le plan Delta se révèle de moins en moins efficace, les tempêtes et la surcote qui leur est associée ne sont plus maîtrisables. Les populations vulnérables, principalement dans la plaine centrale, du delta de l'Escaut à Amsterdam sont contraintes à un retrait précipité. Les événements météorologiques extrêmes de plus en plus fréquents s'accompagnent d'importants dégâts.

Malgré les pressions naturelles croissantes sur leur modèle de développement urbain et agricole, le pays peine à sortir d'une gouvernance spécialisée sur la seule gestion de l'eau. Les relocalisations de villes ou d'infrastructures, comme une partie du port de Rotterdam, ne sont faites qu'en cas de danger récurrent avéré. Mais la stratégie reste fondée sur des réponses technologiques plutôt que sur des restructurations territoriales d'ampleur.

\section{IScénario 2 : Réaménagement du littoral}

Les Pays-Bas s'impliquent fortement dans la lutte contre le changement climatique, en synergie avec les pays européens. De plus, l'Union européenne développe massivement tous les outils de l'adaptation, notamment sur le littoral. L'adaptation à la montée de la mer se développe en appliquant une gouvernance démocratique de l'eau à toutes les échelles territoriales pertinentes et en mobilisant l'ensemble des acteurs concernés. 
La stratégie d'adaptation a évolué : retrait planifié des habitats et des activités humaines de la ligne de côte, constitution de zones-tampons sur les littoraux et dans la plaine centrale, renforcement d'infrastructures de protection naturelles et de barrages/digues plus en retrait. Cette adaptation se fait dans le cadre d'une coopération régionale avec la France, la Belgique, et l'Allemagne pour mieux réguler les débits des trois fleuves Rhin, Meuse et Escaut.

La relocalisation des populations s'accompagne d'un réaménagement urbain, afin de réduire la vulnérabilité des grandes villes de la plaine basse centrale (principalement le triangle Rotterdam-La Haye-Amsterdam), au profit du développement des villes de petite taille, sur les plateaux situés à l'est. Cette trajectoire s'appuie sur la forte culture démocratique de la gouvernance de l'eau qui est ici en capacité d'intégrer une diversité d'acteurs dans des changements radicaux de développement (par exemple, l'impact de la transition énergétique sur l'agriculture et les transports, l'acceptation du repli des habitations et infrastructures...), et d'inscrire la maintenance des infrastructures sur le long-terme, en accord avec les populations. L'expertise acquise dans ces aménagements d'envergure est valorisée au plan international, en Europe, mais aussi en Asie. 


\section{Le Vietnam}

\section{Limportance des deltas au Vietnam}

Le Vietnam eSt un PAyS du sud-est asiatique de $331000 \mathrm{~km}^{2}$ comptant quatre pays voisins : Cambodge, Chine, Laos et Thaïlande. Il s'étire sur 1500 km, la plaine côtière étroite étant adossée à une chaîne de montagnes, sauf au nord, dans le delta du fleuve Rouge et au sud, dans le delta du Mékong. Il connaît un climat tropical à subtropical selon la latitude et est soumis à un régime de mousson, avec des précipitations abondantes (1,2 à $3 \mathrm{~m} / \mathrm{an}$ ). C'est un pays riche d'histoire, constitué en royaume en 2500 avant notre ère. Depuis 1975, le parti communiste structure la vie politique, économique et sociale de l'ensemble du pays. La population (97 millions d'habitants en 2018), en croissance lente, est concentrée sur la bande côtière et dans les deux grands deltas, au nord celui du fleuve Rouge, et au sud, celui du Mékong. À dominante encore rurale, elle s'urbanise : de $13 \%$ en 1995 à $35 \%$ en 2018. L'économie, dite " socialiste de marché », est en croissance rapide ( 5 à $8 \%$ par an depuis 30 ans) avec un PIB de 245 milliards $\$$ (35 rang mondial en 2018). Elle se développe surtout dans les grandes villes des deux deltas. Le secteur majeur est celui des services (51\%) suivi par l'industrie (33\%), puis l'agriculture et la pêche (valeurs 2018). Le PIB/habitant a progressé de 600 à 2200 \$/an entre 1985 et 2018 avec un indice de développement humain de 0,7 , en progression.

\section{Axes d'analyse}

La problématique de la montée du niveau de la mer au Vietnam peut être abordée sous les angles de l'environnement, du social (au sens large) et de l'économie, en tenant compte de leurs interactions.

\section{Au plan environnemental}

Le changement climatique constitue l'une des composantes de la montée du niveau de la mer dans le delta du Mékong. Celle-ci est la résultante de quatre autres phénomènes ou processus :

- la multiplication du nombre de barrages sur le Mékong en amont du pays (10 fleuve du monde par sa taille, soit $4350 \mathrm{~km}$ ) en Chine, mais aussi au Myanmar, en Thaïlande, au Laos et au Cambodge ;

- la réduction des apports en sédiments, d'où le recul du trait de côte du delta, autrefois en extension. Entre les années 1970 et la période 2000-2016, la réduction des apports a été de 75 \% pour le Mékong et de 90 \% pour le fleuve Rouge (Vinh et al., 2014 ; Ha et al., 2018). Les causes sont connues : les pompages dans les sols du delta à des fins agricoles (irrigation), urbaines (eau douce) ou énergétiques (gaz, pétrole) (Minderhoud et al., 2020) ; 
- les extractions de sables et graviers pour la construction (estimés a minima à 55 millions de t/an), ce qui entraîne une érosion accrue des berges et le recul du trait de côte. Une des conséquences de ces phénomènes est la dégradation de la biodiversité dans le fleuve, surtout en aval, en raison des barrages qui bloquent les espèces migratrices et « segmentent » artificiellement les populations de la faune aquatique.

La submersion est observée dans le delta du Mékong, dans certaines baies et lagunes de la côte centrale et dans la région nord Haïphong - Hanoï. L'enjeu premier est celui du Mékong, en raison de la densité urbaine et de l'importance agricole (riz) et économique de cette région.

Les estimations de la hausse varient selon les études et ne cessent d'augmenter. La hausse relative annuelle varie de 0,3 à $2,2 \mathrm{~cm}$ par an dans le delta, ce qui est considérable, car la hausse moyenne de la mer n'est que de $0,3 \mathrm{~mm} /$ an en moyenne depuis 2004 (Dieng et al., 2017). Cet écart s'explique par la subsidence (abaissement du niveau du sol) qui compterait pour 80 à $90 \%$ de ce bilan. Sur la période 1995-2010, on observe une baisse du niveau des nappes phréatiques de $30 \mathrm{~cm}$ par an et, selon les mesures par satellite, une subsidence moyenne de 1,6 cm/an (de 1 à $4 \mathrm{~cm}$ ), (Erban et al., 2014), voire $6 \mathrm{~cm} /$ an (Dörr et al., 2020).

Même une hausse limitée à 1 m en 2100 entraînerait une submersion de la moitié du delta et concernerait environ 17 millions de personnes. Le cas du delta du fleuve Rouge, au nord du pays, est tout aussi préoccupant, bien que l'échelle soit moindre. Enfin, une grande partie des infrastructures de la mince plaine côtière qui va du nord au sud devra, tôt ou tard, être déplacée, et ce en raison des impacts des événements climatiques extrêmes.

Ces phénomènes s'observent dans presque tous les deltas du monde. Les 26000 km² $^{2}$ de deltas déjà situés sous le niveau de la mer et les $96000 \mathrm{~km}^{2}$ situés à moins de $2 \mathrm{~m}$ d'altitude devraient doubler en surface d'ici 2100. La généralisation à l'échelle mondiale d'un déficit d'apport d'eau et de sédiments à la côte risque de s'aggraver avec une série de conséquences négatives pour les écosystèmes comme pour les sociétés humaines (Syvitsky et al., 2009).

\section{Au plan politique (gouvernance), sociétal et de l'urbanisme}

Il apparaît une prise de conscience progressive des enjeux multiples induits par la hausse du niveau de la mer et par les événements météorologiques extrêmes dont la puissance et la fréquence devraient s'accroître (tempêtes, mousson...). Les villes et les infrastructures du delta devraient être inondées plus fortement (+10 à $+70 \mathrm{~cm}$ de 2030 à 2050) et plus fréquemment (de 72 à 365 jours de 2030 à 2050) y compris dans des villes comme Can Thô (1,1 million d'habitants à o,8 m d'altitude) (Nguyen et al., 2016). Dans la région de Hai Hau (côte sud-ouest du delta), les $33 \mathrm{~km}$ de digues de protection ne devraient pas tenir au-delà de 2060, quel que soit le scénario de changement, la menace venant surtout de l'érosion côtière. Celle-ci s'accroît en raison de la disparition progressive des mangroves sur le littoral du pays. 
Selon les provinces concernées, les interlocuteurs et le contexte, les autorités oscillent entre :

- le déni : «Les observations restant dans la variabilité globale, il n’y a pas de tendance alarmante »;

- la discrétion : «On sait qu’il se passe quelque chose, mais il ne faut pas inquiéter les populations »;

- la modération : « Il y aura des impacts de la hausse du niveau de la mer, mais quelques ouvrages adaptés et une bonne information de la population devraient permettre de gérer ce problème »;

- la planification d'actions d'ampleur comme la «fermeture » de deux bras du Mékong afin de recharger en eau douce les bras restants, au nord (Ho-Chi-Minh-Ville) et vers le sud (Can Thô) ;

- la préoccupation : «Il faudra se préparer à des migrations de population, car les impacts de la submersion pourraient menacer de plus en plus de populations pauvres, les plus exposées ».

\section{Au plan économique}

Le plus grand impact potentiel concerne la riziculture dans le delta du Mékong (50\% du total national) et la salinisation pourrait la réduire de $25 \%$ d’ici à 2050 . Cette salinisation est en partie due à l'intrusion saline consécutive à l'extraction de sable qui peut atteindre de 10 à 15 cm/an dans certains lits (Erban et al., 2014). Cette salinisation favorise la transformation de rizières en bassins d'élevage de crevettes et d'espèces euryhalines, comme cela a été observé en Égypte avec des contraintes similaires (Sadek, 2013). Ainsi les surfaces en aquaculture couvrent désormais près de $20 \%$ de la surface du delta (Li et al., 2020) et le delta du Mékong produit $60 \%$ de l'aquaculture nationale (poisson et crevette). Si la hausse atteignait le mètre, la plupart des activités du delta seraient impactées et il faudrait restructurer en profondeur villes, infrastructures et activités.

\section{Synthèse d'études récentes}

LeS nombreuses ÉtUdes mènent à des conclusions diverses, ce qui reflète l'ambivalence des approches, entre volonté de lucidité et préférence d'aménagement minimal. Ces études visent un horizon assez court $(2030,2050)$ pour un pays de 100 millions d'habitants avec une forte densité sur nombre de zones vulnérables. Peu d'études prospectives sur ce phénomène au Vietnam assument que préparer l'avenir implique des restructurations profondes et coûteuses. De fait, le sujet est considéré comme sensible (Bravard et al., 2013). La priorité est donnée à la gestion des problèmes et des crises, au passage de la riziculture à l'aquaculture, à l'adaptation locale et conjoncturelle aux inondations chroniques. De 2005 à 2017, le delta du Mékong a déjà enregistré un solde migratoire négatif de plus d'1,1 million d'habitants (Nguyen et al., 2021). Ni dans les sphères dirigeantes, ni 
au niveau des populations vulnérables, les mentalités ne semblent préparées à un repli stratégique d'ampleur. L'organisme intergouvernemental chargé de proposer des solutions pérennes aux problèmes liés au fleuve (Mekong River Commission) n'a aucun pouvoir contraignant et peu de moyens. Et la Chine, qui exerce la plus forte pression sur le fleuve, refuse d'en faire partie.

\section{Irois scénarios plausibles}

Les trois scénarios partent d'une même évolution climatique mondiale « modérée », soit une hausse de $30 \mathrm{~cm}$ en 2050 (hors subsidence locale) et de $100 \mathrm{~cm}$ en 2100, avec une pente d'évolution positive à la fin du siècle et des événements climatiques extrêmes plus fréquents et plus forts. La vitesse de subsidence dans les deux deltas du pays est variable selon les choix politiques et stratégiques des pays des deux bassins versants du Mékong et du fleuve Rouge.

\section{Scénario 1 : Prise de conscience et pro-action}

La prise de conscience de la vulnérabilité croissante du sud-est asiatique à la submersion marine progresse dans tous les pays de la région, y compris en Chine. Les pays riches et peu vulnérables à cette menace, notamment les puissances régionales (Australie, Singapour, Brunei...) savent qu'une déstabilisation de toute la zone côtière de la région par une submersion, même partielle, aurait des conséquences graves pour leur économie et leur sécurité : exodes de population, pertes d'infrastructures, déstructuration de l'agriculture et du tissu urbain sur la côte, menaces sur la sécurité alimentaire... Cette prise de conscience conduit à une nouvelle impulsion dans la coopération internationale, notamment à l'échelle des pays impliqués dans la gestion des bassins versants du Vietnam. La Mekong River Commission, que la Chine rejoint, est active, reconnue, et dotée de moyens de contrôle et d'incitation. Une politique commune en matière de prélèvements de tous types, d'aménagement des fleuves (barrages, débits réservés...) et d'usage des terres (reforestation, planification des cultures...) permet de freiner la subsidence et de l'annuler en 2100.

La hausse reste toutefois trop forte pour maintenir un statu quo des activités et des habitats. Mais la concertation internationale et l'information des populations permettent la planification d'un repli stratégique progressif, voire d'une émigration négociée avec les pays voisins et coresponsables de la situation. Les provinces limitrophes des pays voisins accueillent des millions de migrants sous statut d' "éco-réfugiés ", statut élaboré par les Nations Unies pour réduire les risques de rejet, marquer le caractère exceptionnel et réversible de la situation et soutenir les pays d'accueil.

Ce repli stratégique de grande ampleur justifie des efforts de recherche et d'innovation technologique pour mieux mesurer, modéliser et anticiper les changements à venir et choisir les meilleures stratégies d'adaptation. De nombreux pays sont ainsi sollicités, notamment en Europe... Une forme de solidarité régionale émerge, avec un partage des 
expériences positives, ainsi qu'à l'échelle mondiale pour des régions aussi vulnérables : golfes du Mexique et de Guinée, Méditerranée, Baltique... Cette évolution « vertueuse » n'est compatible qu'avec la mise en place d'une gouvernance mondiale éclairée, active et dotée de moyens proportionnels aux enjeux considérables liés à la hausse de la mer à l'échelle mondiale.

\section{Scénario 2 : Crises récurrentes et adaptation incrémentale}

La lenteur de la prise de conscience de la menace que fait planer l'association de la hausse du niveau de la mer et de la subsidence d'origine anthropique conduit à des situations critiques pour un nombre croissant de régions côtières dans le pays. Les autorités reconnaissent peu à peu les dangers liés à la submersion marine et à la salinisation des terres. Les pressions pour passer à l'action viennent des populations concernées et des organisations internationales confrontées à des problèmes identiques dans d'autres pays voisins comme la Thaïlande, l'Indonésie et les Philippines.

Plusieurs tempêtes entraînent des pertes de bande littorale et de villages entiers. Les autorités prennent alors des mesures conjoncturelles comme la construction de digues tout en reportant les décisions les plus coûteuses, comme le repli à grande échelle des infrastructures et des populations exposées. Une catastrophe d'ampleur finit par décider le gouvernement à lancer un équivalent du « plan Delta » hollandais, combinant tous les outils d'aménagement classiques (digues, barrages mobiles, réseaux de pompage, restauration de mangrove...) et l'aide internationale.

Même tardives, ces réactions finissent par porter leurs fruits et des portions de zones côtières sont mises en sécurité. Cependant, certaines régions sont abandonnées, comme celles situées en aval des deux bras centraux du Mékong afin de répartir l'eau douce dans les zones les plus peuplées. Mais les plans d'urgence se succèdent sans solution pérenne. Il faut donc replanifier tous les 10 ans des programmes de repli. Cette situation de précarité conduit à l'ouverture de négociations, difficiles, avec les pays situés en amont des deux deltas. La lenteur de la prise de décision s'accompagne de la poursuite de la subsidence. La hausse du niveau de la mer concerne des espaces de plus en plus vastes. Cette vulnérabilité croissante n'est compensée que par à-coups, sans plan de restructuration globale à moyen terme.

\section{Scénario 3 : Déni persistant et catastrophes}

La position de scepticisme des autorités en matière de risques de submersion marine persiste pendant plusieurs décennies. Le gouvernement tente de minimiser le phénomène et certains lanceurs d'alerte, découragés, finissent par quitter le pays. La population est partagée entre inquiétude et résignation. Les coopérations en appui aux programmes de protection et de prévention sont limitées aux sites les plus exposés et les négociations internationales sur la gestion des deux fleuves ne progressent pas. En effet, les États voisins privilégient d'abord leurs propres intérêts, notamment la Chine qui maintient ses capacités de pression sur le Vietnam, notamment en vue de poursuivre sa politique 
régionale d'expansion territoriale en mer. L'Association des nations de l'Asie du Sud-Est (ASEAN) et le forum de la coopération pour l'Asie-Pacifique (APEC) fonctionnant mal, et l'absence de dialogue régional conduit à des stratégies politiques strictement nationales. La Mekong river Commission constate les dysfonctionnements du fleuve, mais elle reste impuissante. Face aux menaces de submersion, les pays voisins suivent leur logique propre de défense côtière et d'aménagement du littoral et des zones basses. Les entreprises étrangères proposent des solutions pour chaque pays mais sans plan coordonné à moyen terme.

La situation s'aggravant au fil des décennies, une partie des classes aisées et les jeunes diplômés sont tentés de partir à l'étranger, tandis que les populations côtières vulnérables cherchent à s'exiler par la mer pour trouver refuge en Indonésie, en Malaisie et jusqu'en Australie. La bande côtière littorale est impactée, ce qui aboutit à une coupure récurrente des échanges entre le nord et le sud.

Le pays affronte alors la pire conjoncture : l'impuissance des décideurs, l'absence de politique structurelle efficace, le retard des investissements vitaux en infrastructures de sécurité civile, la baisse de la sécurité alimentaire due à la régression des surfaces rizicoles et la fuite des compétences. Le seul recours est l'armée qui doit gérer des situations de crises à répétition et encadrer des relocalisations forcées. Ce repli mal préparé est source de tensions, voire de violences sociales. Cette évolution conduit à une situation de survie du pays qui se trouve alors contraint d'importer des denrées vitales. Les Nations Unies doivent créer et gérer des camps de réfugiés sur les zones frontalières de l'ouest, la frontière chinoise étant fermée. Les pays d'accueil potentiels de la région comme l'Australie, ne veulent pas accueillir des millions de réfugiés, sachant qu'ils risquent de rester pour toujours. Sans prise de conscience et réaction à grande échelle, l'impasse paraît complète.

\section{Conclusion}

En ADMetTANT QUe Les CAuses de la subsidence des deltas vietnamiens soient maîtrisées d'ici 2100, on devrait observer, tout d'abord une aggravation de ce phénomène, puis une diminution, en fonction des progrès de la coopération internationale des pays concernés, pour atteindre l'équilibre en 2100 (voir fig. 5). Cette évolution devrait atteindre des valeurs maximales de hausse vers 2060 pour diminuer jusqu'à s'annuler en 2100. À la fin du siècle, la hausse apparente du niveau de la mer devrait retrouver la courbe de hausse générale moyenne mondiale. Ce cumul de la subsidence et de la hausse naturelle conduit à atteindre un mètre vers 2050-2060, soit 40 à 50 ans avant le reste du monde, selon l'hypothèse haute actuelle du GIEC. En conséquence, même avec l'hypothèse de maîtrise de la subsidence en 80 ans, le Vietnam devrait connaître des épisodes de submersion sévère dès les années 2030-2040. En cas de non-maîtrise de la subsidence, et d'accélération de la hausse moyenne de la mer, l'hypothèse d'une hausse cumulée à 2 m 
en 2100 ne peut pas être écartée. Elle aboutirait à des situations catastrophiques sur tous les plans. L'aménagement de la majeure partie du pays serait alors à « repenser », surtout si la hausse de la mer se poursuit au xxı ${ }^{\mathrm{e}}$ siècle. Même une maîtrise rapide de la subsidence, en trois ou quatre décennies, permettrait d'allonger le délai d'adaptation du pays, sans le mettre à l'abri toutefois d'une profonde restructuration, indispensable à terme.

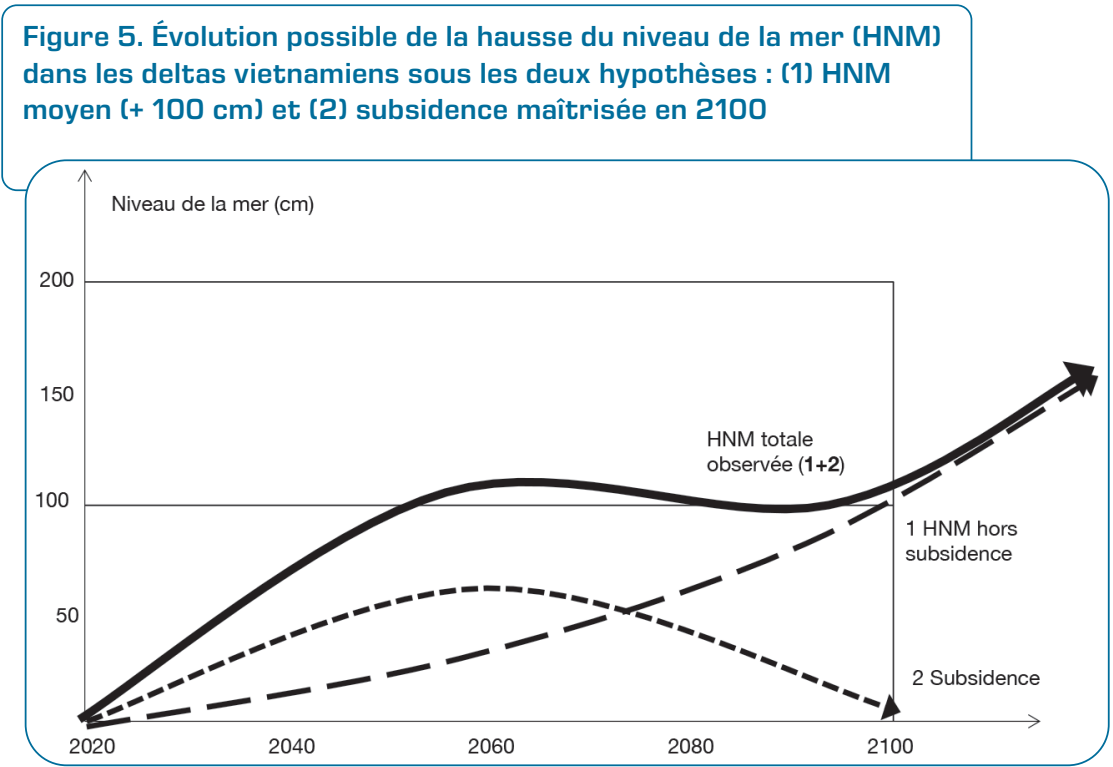

Le tableau 3 montre la combinaison de trois hypothèses de subsidence et de hausse dans les deux grands deltas vietnamiens. Les cumuls montrent que la valeur considérée comme « critique » de $1 \mathrm{~m}$ serait atteinte, voire dépassée dans 7 cas sur 9. Ce tableau montre l'intérêt de considérer sérieusement ce qu'impliquerait la prise en compte du principe de précaution dans la sécurisation des zones basses du Vietnam.

Tableau 3. Croisement de 3 hypothèses de subsidence et de 3 hypothèses de hausse du niveau de la mer (valeurs à 2100, sans préjuger de la pente; en gras sur fond gris, valeurs supérieures à 1 m)

\begin{tabular}{lllll} 
& $\begin{array}{c}\text { Hausse moyenne mondiale }(\mathrm{cm}) \\
\text { Subsidence deltaïque }(\mathrm{cm})\end{array}$ & 50 & 100 & 200 \\
\hline 20 & 70 & 120 & 220 \\
\hline 40 & 90 & 140 & 240 \\
\hline 60 & 110 & 160 & 260
\end{tabular}





\section{Partie III}

\section{Conséquences potentielles des scénarios}

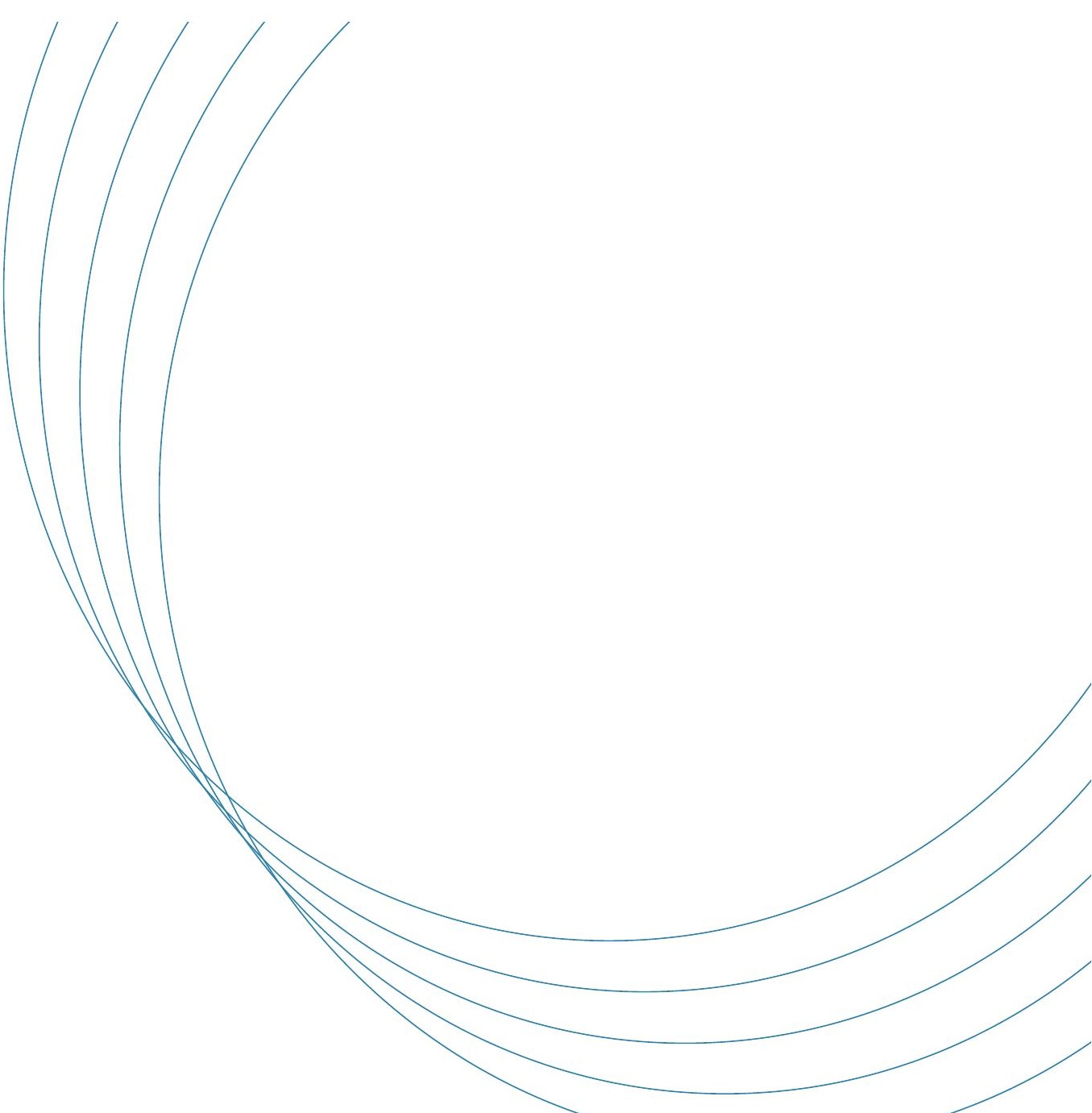




\section{Enyironnement}

Le Mont-Saint-Michel devrait voir s'accentuer son caractère insulaire avec des zones de pré salé en extension vers l'intérieur des terres.

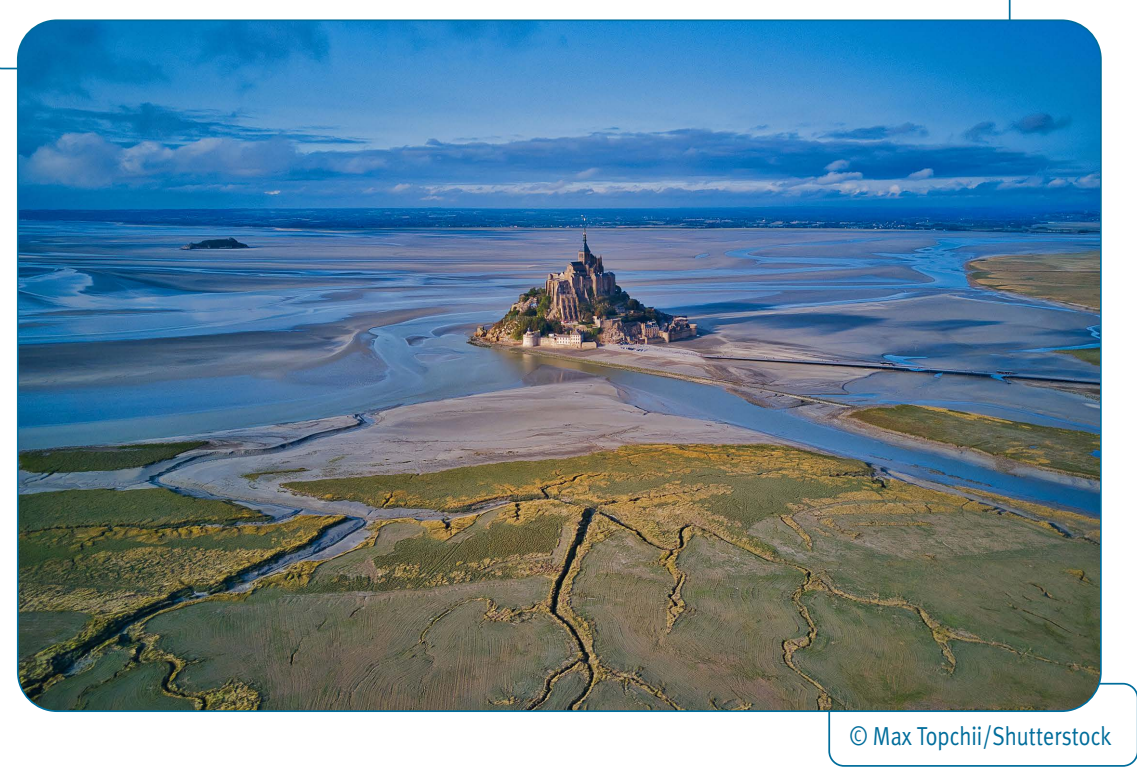

Le recul des côtes à falaise de roche sédimentaire devrait s'accélérer en raison d'une attaque plus directe de la base des falaises par les vagues.

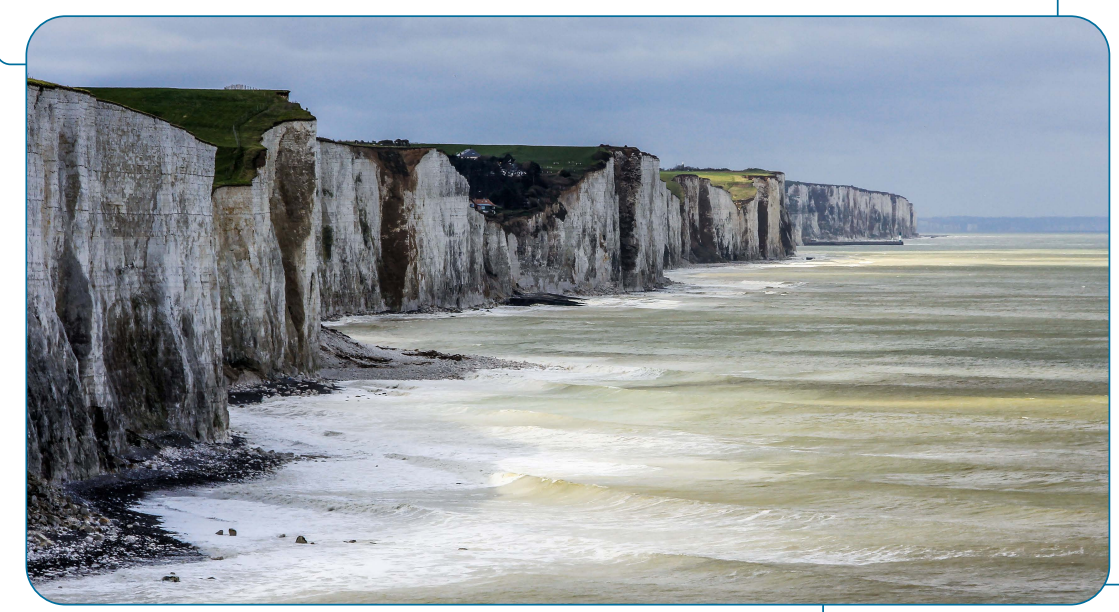

(c) Wilfried Photo/Shutterstock 
Villes

Rotterdam, premier port d'Europe, est de plus en plus menacé, autant par la submersion marine que par les inondations fluviales du Rhin ; avant la fin du siècle, il faudra envisager sa relocalisation.
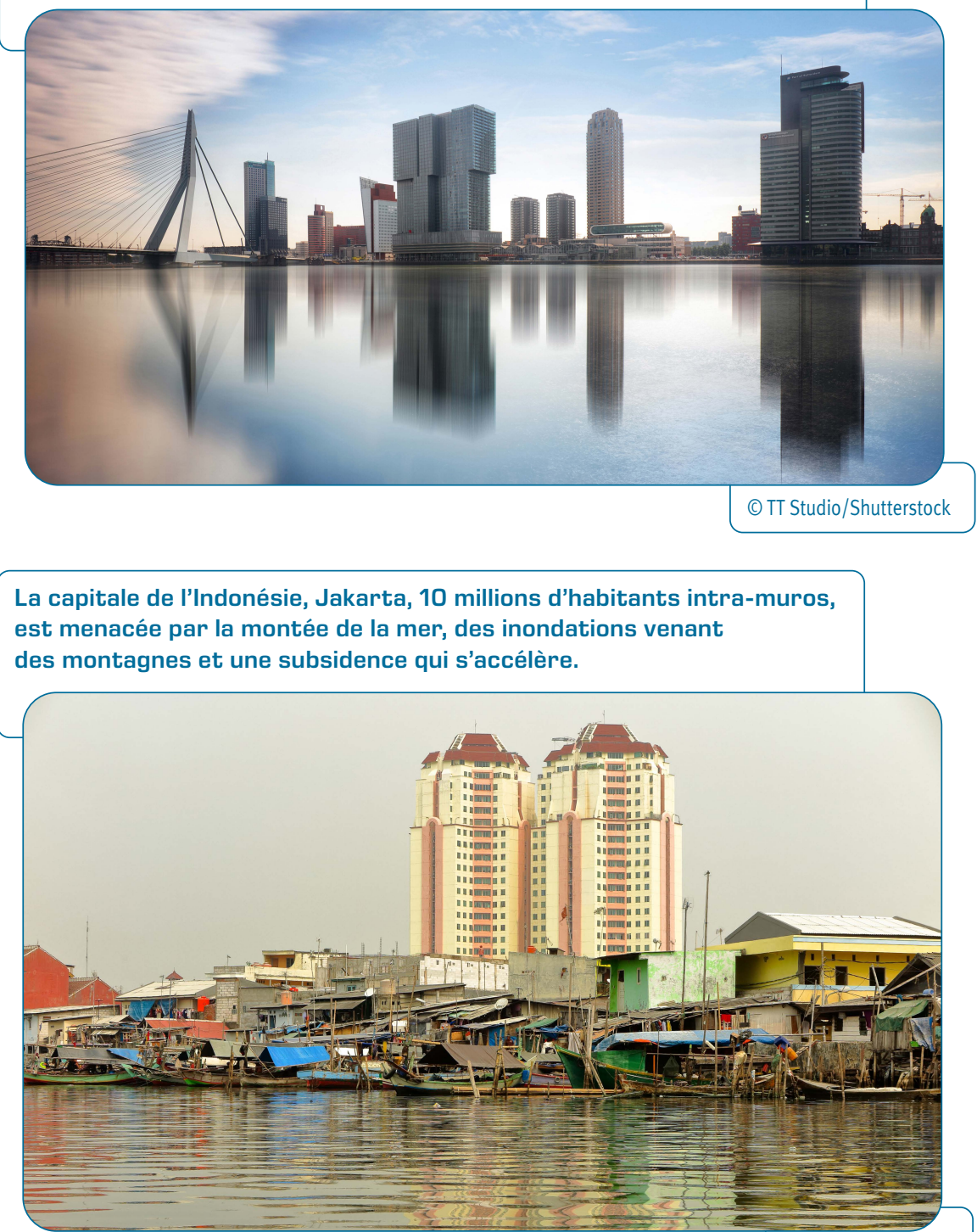
Ville musée, patrimoine de l'Unesco, Venise a déjà bénéficié de grands travaux de protection (plan Moïse) contre les inondations (agua alta) mais sa sauvegarde n'est pas certaine sur le long terme.

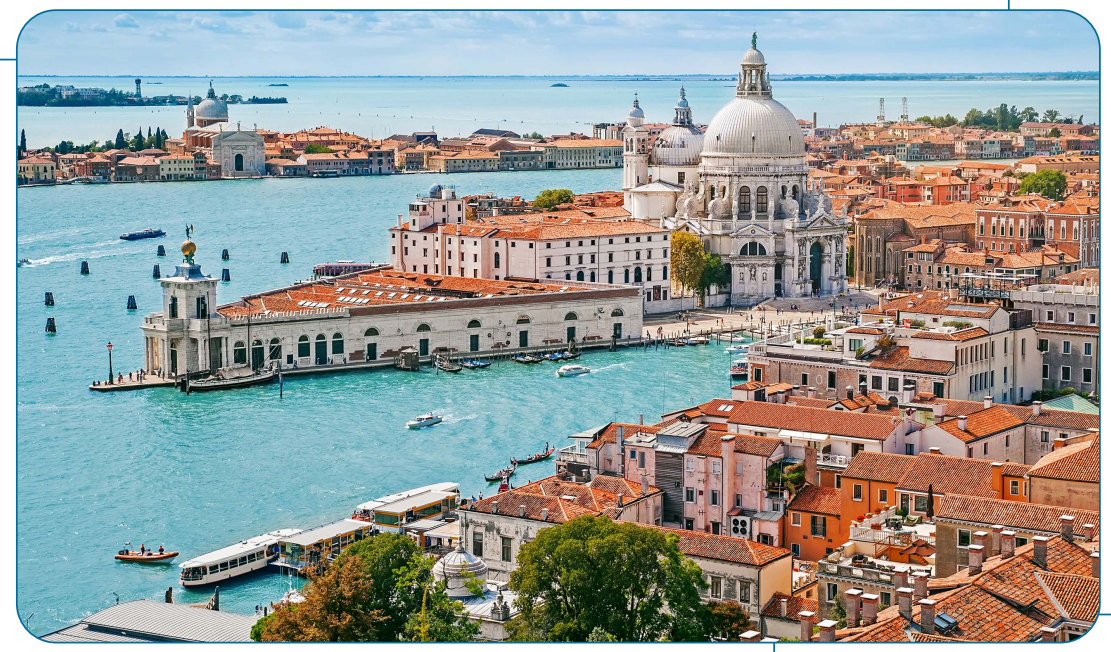

(c) Mariia Golovianko/Shutterstock

Sur de nombreux littoraux africains, les constructions trop exposées à la mer doivent être abandonnées, comme ici, à Yenne Tode, au Sénégal.

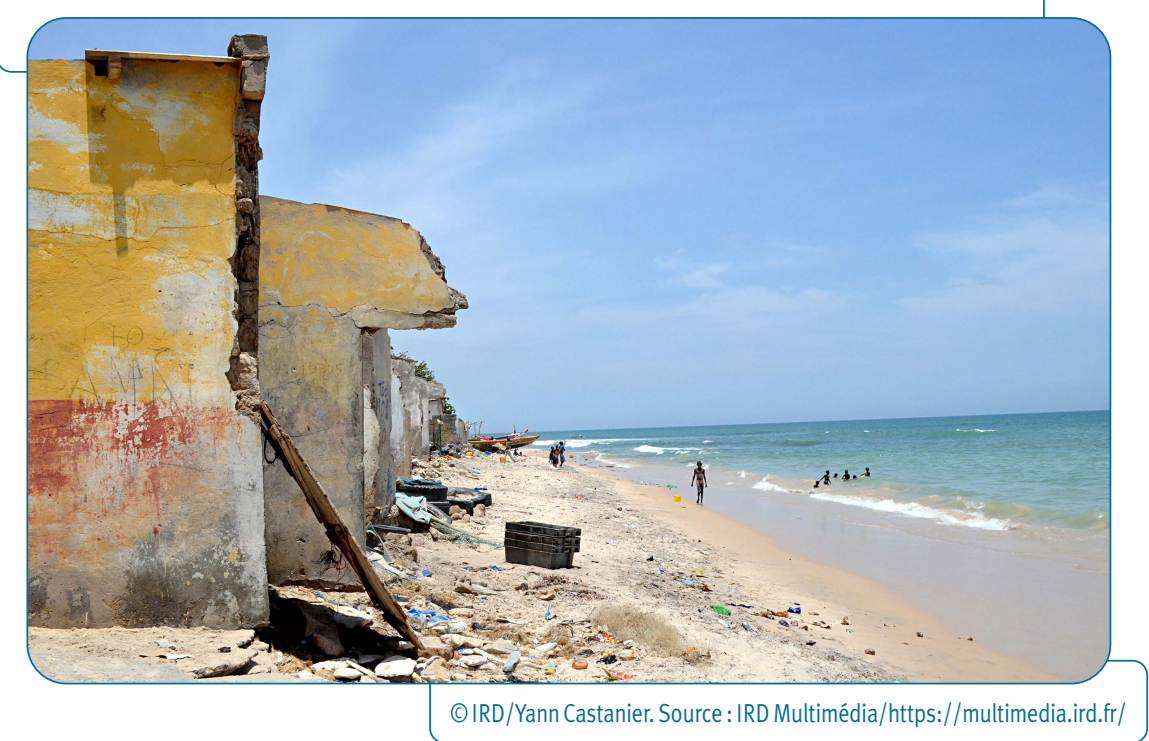




\section{Économie}

De nombreuses infrastructures situées sur le littoral, comme cet aéroport à Gibraltar, devront être protégées, voire être relocalisées face à la montée du niveau de la mer couplée à l'accroissement de la violence des tempêtes.

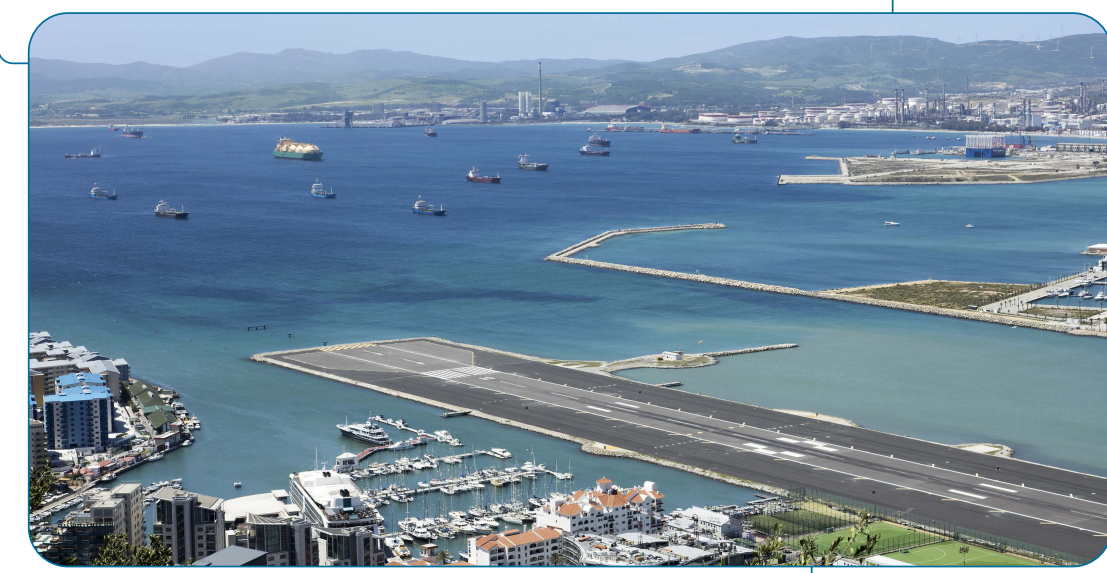

(c) David Wingate/Shutterstock

La relocalisation des infrastructures industrielles côtières vulnérables pose de nombreux problèmes dont celui de la pollution des sols.

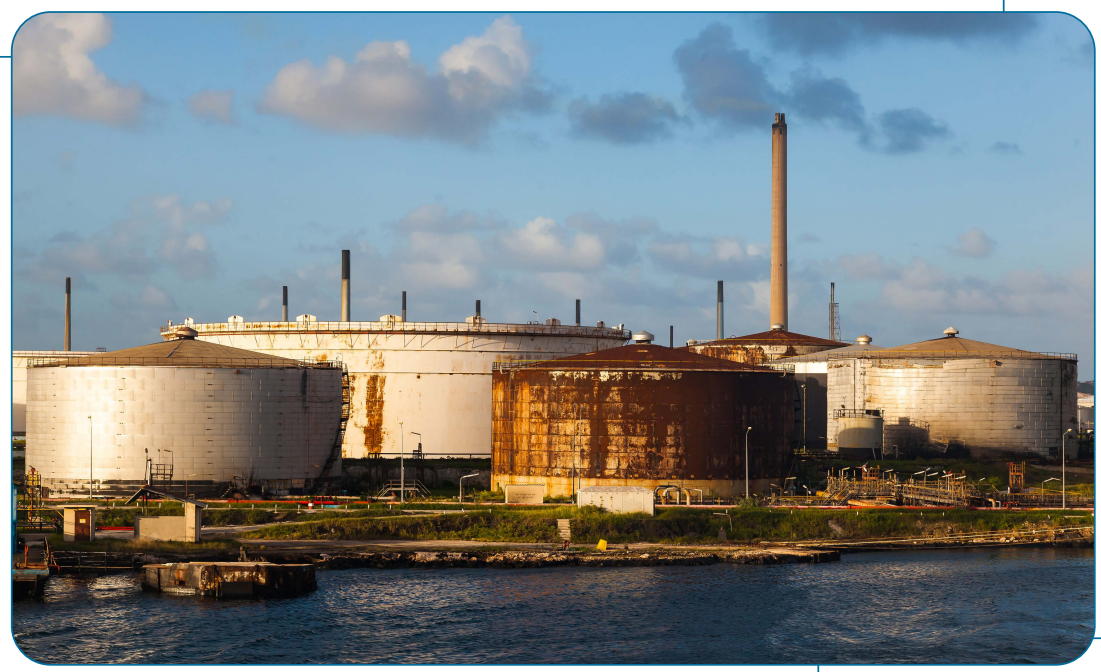

(c) ZHMURCHAK/Shutterstock 


\section{Géo politique}

Oceanix City, projet de cité flottante multifonction et autonome au large.

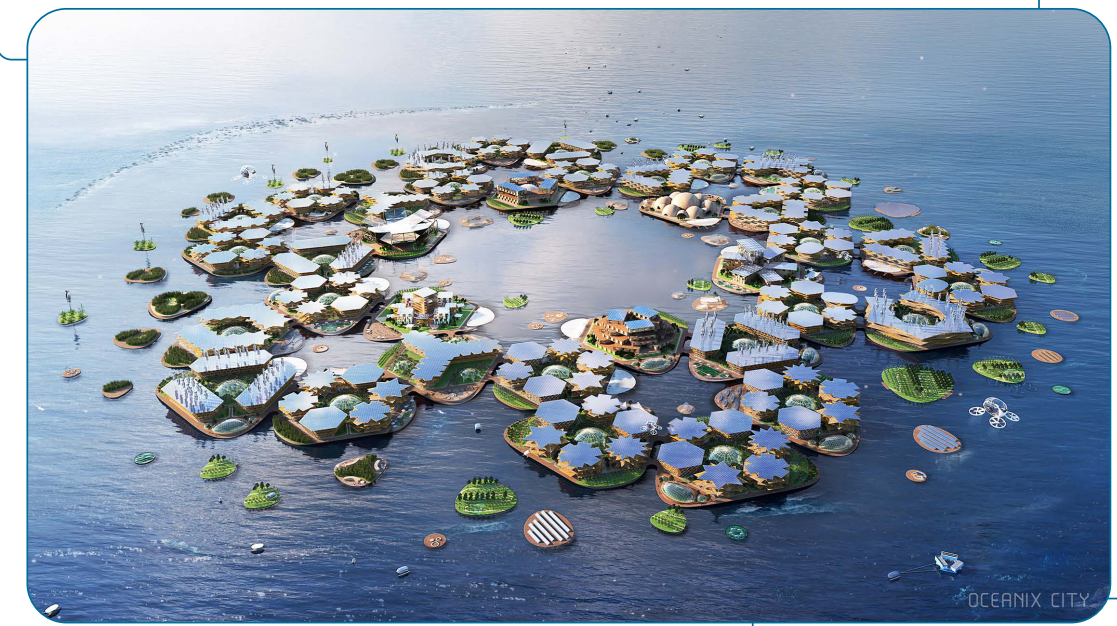

(c) OCEANIX/BIG-Bjarke Ingels Group.

\section{Une solution technologique : l'habitat flottant ;} ici, maisons du quartier d'ljburg d'Amsterdam.

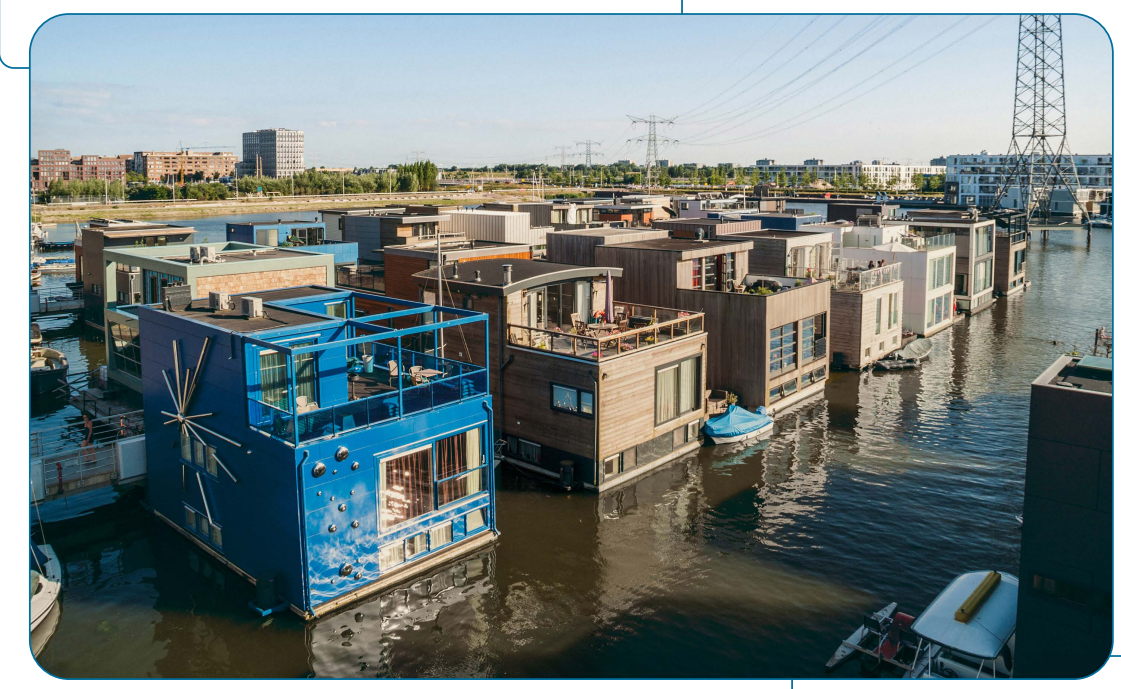

(c) Kleinstofzuigertje/Shutterstock 
Malé, île-capitale des Maldives, dans l'océan Indien, n'a, en moyenne, que $2 \mathrm{~m}$ de hauteur au-dessus du niveau de la mer. Sa restructuration radicale, voire son évacuation partielle, devra être planifiée.

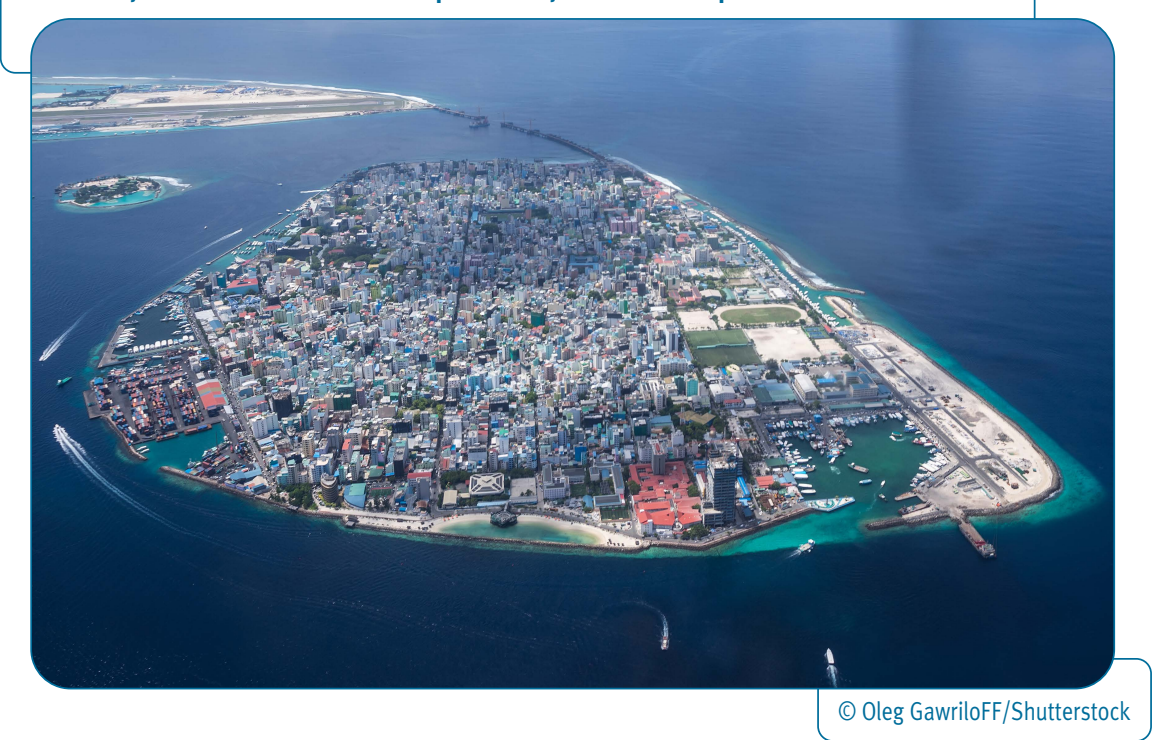




\section{Pour l'environnement et les ressources naturelles}

LES ÉCOSYSTÈmES LITTORAUX ET CÔTIERS abritent une biodiversité très riche tant sur terre qu'en mer et dans les airs et sont plus ou moins sensibles aux conséquences de l'élévation du niveau de la mer. Ils présentent en effet une grande diversité qui se caractérise par : - l'estran, c'est-à-dire la zone de plage (de sable ou de galets) et les vasières qui sont soumises à l'influence des marées ;

- les côtes et falaises rocheuses ;

- les pelouses aérohalines (formations végétales rases dominées par les graminées sur les côtes exposées aux vents dominants) ;

- les cordons dunaires et les zones humides intra-dunaires ;

- les marais maritimes (prés-salés, marais salés endigués, forêts de mangroves tropicales...) ;

- les lagunes côtières ;

- les estuaires des cours d'eau ;

- les lagons récifaux.

L'élévation du niveau de la mer n'est qu'une des pressions (forçage) auxquelles sont soumis ces écosystèmes du fait du changement climatique. Elle s'ajoute notamment au réchauffement et à l'acidification des eaux, ainsi qu'aux conséquences de l'artificialisation croissante des littoraux sous la pression de l'urbanisation et du développement des activités économiques et des infrastructures qui y sont liées. De ce fait, il est difficile de déterminer la part spécifiquement due à l'élévation du niveau de la mer dans la dégradation des écosystèmes littoraux et côtiers. On peut estimer qu'elle est parfois secondaire en comparaison des effets du réchauffement de l'océan et des interventions humaines diverses (Gattuso et al., 2015). Cependant les pollutions par lessivage des zones urbaines et industrielles et la salinisation lors des épisodes de submersion réduisent la biodiversité et favorisent les espèces capables de se développer en milieu salé.

L'évolution du trait de côte est une traduction directe de l'élévation relative du niveau de la mer, de la fréquence et de l'intensité des tempêtes, et de la disponibilité sédimentaire du système littoral. Elle est parfois accentuée par certains prélèvements (sable, galets...), ou ouvrages de protection qui modifient les transports de sédiments par charriage, transport par l'eau ou le vent, et leurs dépôts. La vulnérabilité du trait de côte dépend du contexte géologique et topographique. Elle est accrue par la dégradation, voire la disparition, d'écosystèmes qui constituent eux-mêmes une protection naturelle, tels que les mangroves tropicales ou les récifs coralliens.

Au-delà de la biodiversité, deux ressources naturelles majeures sont directement impactées par l'élévation du niveau de la mer : l'eau douce et le sol. Toutes deux sont susceptibles d'être dégradées par la conjonction de phénomènes de salinisation ou de pollution 
des nappes phréatiques et des sols côtiers. Les sols sont également soumis à l'érosion et au lessivage lors des épisodes de submersion faisant suite aux tempêtes.

\section{Les écosystèmes littoraux}

LES RÉCIFS CORALLIENS SONT, avec les forêts tropicales, les écosystèmes les plus riches de la planète. Couvrant $600000 \mathrm{~km}^{2}$, soit 0,2 \% de la surface des océans, ils abritent le quart de toutes les espèces marines connues dans le monde, soit environ 100000 espèces : coraux, poissons, mollusques, tortues, requins, etc. Les récifs coralliens, qui dissipent 97 \% de l'énergie des vagues, sont menacés par les conséquences du changement climatique (réchauffement et acidification des océans) et l'élévation du niveau de la mer à un rythme supérieur à la croissance du corail : 20 \% des récifs coralliens sont d’ores et déjà perdus et 25 \% gravement menacés (Burke et al., 2011). En Australie, la Grande Barrière de corail connaît par endroits un taux de mortalité de $60 \%$. Cependant, il y aurait la possibilité d'une certaine adaptation des coraux à la montée du niveau de la mer (Duvat, 2018). Les mangroves tropicales sont également des écosystèmes très riches et constituent des zones nourricières pour de nombreuses espèces. Pourtant, leur surface a reculé de $19 \%$ dans le monde entre 1980 et 2005 , entraînant une exposition et une vulnérabilité croissante aux aléas marins, du fait de la disparition des protections naturelles des côtes. Outre la construction immobilière, une cause importante en est le développement de l'élevage de crevettes à grande échelle, comme en Indonésie, en Malaisie ou aux Philippines. Dans certaines régions, la mangrove est remplacée à $80 \%$ par des bassins à crevettes ou de la riziculture. Parfois les palétuviers sont simplement coupés pour en faire du bois de chauffage ou de construction. Avec la montée du niveau de la mer, certains arbres ne pourront subsister, alors que la montée en puissance des ouragans et les cyclones justifie de plus en plus l'intérêt de leur fonction protectrice.

Avec près de $60000 \mathrm{~km}^{2}$ de récifs coralliens et lagons, répartis dans la zone intertropicale des trois océans, la France d'outre-mer compte 10 \% de la surface des récifs du monde. S'y ajoutent les écosystèmes associés, mangroves $\left(1000 \mathrm{~km}^{2}\right.$ ) et herbiers (plus de $1200 \mathrm{~km}^{2}$ ). Dans les estuaires, l'élévation du niveau marin est reproduite intégralement, voire amplifiée, sur l'ensemble du linéaire de l'estuaire et se traduit par un déplacement vers l'amont des zones de nourriceries des espèces marines migratrices sous l'effet de la remontée de la salinité dans l'estuaire. L'amortissement de la propagation de l'élévation du niveau marin en estuaire est principalement régi par le débit : plus le débit est important moins le niveau marin se propage dans l'estuaire.

La dynamique d'évolution des écosystèmes littoraux et côtiers va dépendre à la fois de la vitesse des changements permettant ou non leur adaptation et, dans certains cas, de leur situation géographique permettant ou non leur déplacement vers l'intérieur des terres en conservant les services écosystémiques qu'ils rendent tels que la fixation de $\mathrm{CO}_{2}$, le filtrage des sédiments, les habitats, la biodiversité et la protection des côtes. Lorsque 
cette adaptation ou cette translation ne sera pas possible les écosystèmes disparaîtront ou seront profondément modifiés et leurs fonctions écosystémiques altérées. Dans ce cas, leur rôle dans la protection du littoral disparaîtra également, ce qui augmentera la vulnérabilité de ce dernier.

\section{L'évolution du trait de côte}

Le trait de côte évolue sous l'effet de processus marins (houle, marées, courants), climatiques (vent, gel, précipitations) et anthropiques (prélèvement de galets ou granulats, barrages...). Il peut donc être aussi profondément modifié. Ceci est particulièrement visible sur la côte atlantique où les blockhaus du mur de l'Atlantique ou certains immeubles construits en bord de mer, tel que Le Signal à Soulac-sur-Mer (33), constituent de bons marqueurs de l'avancée de la mer. Les tempêtes fragilisent également les dunes littorales et les falaises. Ainsi, $24 \%$ du littoral français métropolitain recule chaque année, quand $45 \%$ est stable, $11 \%$ gagne des terres, et les $20 \%$ restants sont des terres artificialisées donc fixées (IFEN, 2006). L'action originale mise en place en France via le Conservatoire du littoral permet de sauvegarder certains écosystèmes littoraux des pressions anthropiques et facilite la mise en œuvre d'une gestion adaptée aux évolutions « naturelles ». En Aquitaine, le trait de côte devrait reculer de $50 \mathrm{~m}$ en côte sableuse et de $27 \mathrm{~m}$ en côte rocheuse d'ici 2050 (Bernon et al., 2016). Le Conservatoire national du littoral estime que $2 \%$ des espaces qu'il gère vont disparaître, $78 \%$ vont rester stables et $20 \%$ seront soumis à une submersion temporaire. Plusieurs stratégies sont possibles : suivre l'évolution naturelle, accompagner le processus naturel avec des aménagements légers, organiser le repli sur une nouvelle ligne de côte, maintenir à tout prix le trait de côte par des ouvrages de génie civil (défense). Les choix stratégiques à réaliser dépendent cependant de l'intensité et de l'étendue du phénomène et des connaissances permettant de les prévoir et de les anticiper suffisamment à l'avance.

\section{La ressource en eau}

L'eau sur terre est composée à 97,5\% d'eau salée et de seulement 2,5\% d'eau douce, qui se décompose en 1,8\% de glace et de neige, et $0,7 \%$ d'eaux douces de surface ou souterraine. L'eau douce est donc une ressource naturelle rare d'autant qu'elle est très inégalement répartie et que sa disponibilité à tendance à se réduire, à la fois du fait du réchauffement climatique et des pollutions (agricoles, industrielles ou urbaines) qui peuvent la rendre impropre à certains usages tels que l'eau potable ou l'agriculture. Dans le même temps, les besoins augmentent du fait de l'accroissement de la population mondiale et de l'élévation des niveaux de vie. Les prélèvements d'eau annuels au niveau mondial ont été multipliés par plus de 7 entre 1900 et 2000 pour atteindre $4000 \mathrm{~km}^{3}$. Ils devraient atteindre $10000 \mathrm{~km}^{3}$ avant la fin du siècle. Par conséquent, le volume d'eau douce de qualité disponible par habitant diminue régulièrement. Selon les normes de l'OMS, le Maghreb, l'Afrique saharienne, l'Afrique australe et le Moyen-Orient 
sont d'ores et déjà en situation de pénurie (<1 $000 \mathrm{~m}^{3} / \mathrm{hab}$./an), l'Inde, l'Iran et l'Europe centrale sont en situation de stress hydrique ( $<1700 \mathrm{~m}^{3} / \mathrm{hab}$./an) et la Chine en situation de vulnérabilité. La Californie ou l'Australie sont d'ores et déjà soumises à des sécheresses de plus en plus fréquentes.

Ceci est particulièrement vrai dans la grande zone côtière où se concentre 60 \% de la population mondiale, avec parfois de fortes variations saisonnières, la population pouvant être multipliée par 10 en été dans certaines stations balnéaires, faisant coïncider les plus forts besoins en eau avec la disponibilité de la ressource la plus faible. En France, les communes littorales accueillent un peu plus de $10 \%$ de la population sur seulement $4 \%$ du territoire et disposent d'une capacité totale d'accueil de près de 8 millions de personnes. Les efforts de sobriété et de recyclage engagés par l'industrie et par l'agriculture, premier secteur de consommation d'eau, n'empêchent pas des conflits d'usage de plus en plus fréquents.

Sur les littoraux cette ressource est souvent polluée par les activités industrielles et les fleuves qui charrient les pollutions diffuses agricoles (nitrates, phosphore, pesticides...) et urbaines, conduisant à des proliférations d'algues, et les polluants émergents qui ne sont pas traités par les stations d'épuration. Les tempêtes de plus en plus fréquentes et violentes sont également à l'origine de submersions de la zone littorale qui entraînent des pollutions par lessivage. La pénétration du biseau salé dans les nappes phréatiques côtières et dans les estuaires, accentuée par l'intensité des prélèvements et la réduction des débits d'étiage, conduit à une salinisation croissante des eaux côtières avec des conséquences considérables dans de nombreux domaines comme l'agriculture ou le tourisme. Un accroissement de $2{ }^{\circ} \mathrm{C}$ conduirait à passer de $2 \%$ à $10 \%$ de la population mondiale sous le seuil de $500 \mathrm{~m}^{3}$ d'eau douce disponible/an. Or la concentration continue des populations et des activités sur les côtes accroît fortement les besoins en eau douce dans la zone côtière et littorale. La mobilisation de toutes les technologies et l'adaptation des comportements pour optimiser l'utilisation de cette ressource et son efficience s'y imposent plus qu'ailleurs.

Il est certes possible d'accroître la ressource en eau douce sur le littoral par dessalement de l'eau de mer. Les technologies existent (osmose inverse, distillation, électrodialyse, condensation) mais restent coûteuses et consommatrices en énergie ( 2 à $5 \mathrm{kWh} / \mathrm{m}^{3}$ ). Les capacités s'accroissent néanmoins de $10 \%$ par an à l'échelle mondiale, pour moitié en osmose inverse avec un coût moyen de 0,50 \$/ $\mathrm{m}^{3}$. La sobriété, la récupération des eaux de pluie et leur stockage et le recyclage et la réutilisation des eaux usées sont autant de moyens de limiter la tension sur la ressource.

Il est également possible d'envisager d'approvisionner les zones littorales en eau douce par transfert de ressources provenant de l'intérieur des terres via des canalisations en dérivant des fleuves ou par bateau, mais cela ne peut se faire sans impacts écologiques forts sur les régions concernées, sans compter de possibles tensions géopolitiques. 


\section{Les sols}

Les sols sont une ressource naturelle dont la qualité est susceptible d'être directement impactée par l'élévation du niveau marin du fait des phénomènes de salinisation, de pollution par lessivage et d'érosion liés aux épisodes de submersion périodiques lors de tempêtes.

La pression démographique croissante sur les littoraux, et le développement des activités économiques (infrastructures portuaires, zones industrielles ou équipements touristiques) conduit par ailleurs à une artificialisation et à une imperméabilisation croissante des sols, avec des conséquences en termes de fréquence et d'importance des inondations, notamment lors de la coïncidence de marées de fort coefficient avec des précipitations importantes.

À la dégradation de la qualité des sols s’ajoute leur artificialisation croissante en raison de l'augmentation de la pression foncière, de la concentration des populations sur le littoral et du développement du tourisme. Cela contribue à réduire la disponibilité locale des sols pour l'agriculture.

Les hypothèses d'évolution dépendent fortement des actions d'anticipation et de prévention entreprises pour éviter ou réduire les submersions et limiter les pollutions grâce à la dépollution préalable des sites inondables lors des tempêtes. La limitation du pompage dans les nappes en zone basse côtière réduirait la progression du biseau salé et par conséquent la salinisation des sols. 


\section{Pour l'agriculture et l'usage des terres dans les zones littorales}

\section{Les tendances actuelles dans les zones littorales}

ALORS QUE LA POPULATION EST fortement concentrée sur les zones littorales, l'agriculture est plus dispersée dans l'espace. Ainsi, la zone littorale étendue (à moins de $100 \mathrm{~km}$ de la côte et à moins de $100 \mathrm{~m}$ d'altitude), où réside $27 \%$ de la population mondiale, ne concentre que $12 \%$ des terres arables (187 millions d'hectares) et $5 \%$ des pâtures mondiales (167 millions d'hectares) (Kummu et al., 2016). En revanche, les terres arables localisées dans les zones littorales sont souvent irriguées (30\%). Ainsi la zone littorale étendue concentre près de $20 \%$ des terres irriguées à l'échelle mondiale (61 millions d'hectares). Les deltas, espaces de production agricole intensive, jouent un rôle essentiel dans l'approvisionnement alimentaire des pays, notamment les deltas du Gange et du Brahmapoutre au Bangladesh, celui de la Volta au Ghana, celui du Mahanadi en Inde, et celui du Nil en Égypte.

Si on adopte une définition plus restrictive de la bande littorale (correspondant aux surfaces émergées à une altitude inférieure à $5 \mathrm{~m}$ ), les terres arables littorales représentent 40 millions d'hectares, et occupent $26 \%$ de la surface littorale ainsi définie (Dasgupta et al., 2011).

Les travaux sur l'évolution des surfaces agricoles littorales à l'échelle globale sur le siècle passé montrent deux tendances distinctes (Kummu et al., 2016). D’une part, on observe une réduction tendancielle des terres arables sur les zones littorales (par rapport à la moyenne mondiale), tandis qu'elles augmentent dans presque toutes les autres zones. Cette réduction résulte principalement de l'artificialisation des sols liée aux dynamiques d'urbanisation littorale et des processus de salinisation de sols. Pour autant, l'intensification de la production agricole semble s'y être accrue plus rapidement que dans les autres zones. Les travaux de Neumann et al. (2011) montrent que les fortes densités de population coïncident avec le développement de terres irriguées. Cette intensification agricole résulte d'une pression foncière accrue du fait de la concurrence pour l'usage des terres. En effet, les fortes dynamiques d'urbanisation dans les zones littorales génèrent une demande croissante de terres pour implanter des infrastructures de transport, des zones d'activités économiques et les logements, au détriment des terres agricoles existantes. 
La salinisation est un facteur de diminution de la disponibilité des terres, qui deviennent impropres à l'agriculture (voir partie précédente).

L'irrigation est également un facteur majeur de subsidence des sols, en particulier dans les zones de delta. La subsidence est liée à deux facteurs majeurs : l'affaissement des sols, notamment sous l'effet du pompage des eaux souterraines pour l'agriculture, et la réduction des apports de sédiments du fait d'un moindre apport des fleuves, lorsqu'il y a des barrages en amont, et de prélèvements accrus de matériaux pour la construction (sables et graviers). Cet affaissement est aussi lié à l'extraction de ressources énergétiques comme le gaz ou le pétrole, dans plusieurs grands deltas comme celui du Mékong (Dang et al., 2018). Dans le cas de ce delta, la subsidence est quatre fois plus rapide que la montée du niveau de la mer. Plus des deux tiers (71\%) des deltas sont affectés par ce phénomène (Syvitski et al., 2009). C'est donc un enjeu majeur pour l'évolution des surfaces agricoles sur les zones littorales qui pourrait être amplifié par l'élévation du niveau de la mer.

\section{L'év olution de la disponibilité des terres agricoles littorales}

Les travaux de Dasgupta et Al. (2009), réalisés sur une base de 87 pays en développement, évaluent les pertes de terres agricoles littorales sous la double contrainte d'une élévation du niveau de la mer de $1 \mathrm{~m}$ et d'une surcote liée à l'intensification des cyclones et des tempêtes. Dans une hypothèse haute, près de 20 millions d'hectares de terres arables pourraient être affectés, soit la moitié des terres agricoles littorales (voir Tableau 4).

Tableau 4. Surfaces agricoles mondiales affectées par une élévation du niveau de la mer et une surcote [estimation d'après table 2 dans Dasgupta et al. (2009)]

\begin{tabular}{lccc}
$\begin{array}{c}\text { Contexte d'élévation et de surcote } \\
\text { (HNM : hausse du niveau de la mer) }\end{array}$ & $\begin{array}{c}\text { Surface agricole } \\
\text { impactée } \\
\text { (en millions } \\
\text { d'ha) }\end{array}$ & $\begin{array}{c}\% \text { des surfaces } \\
\text { agricoles littorales } \\
\text { (total : } 40 \text { millions } \\
\text { d'hectares) }\end{array}$ & $\begin{array}{c}\% \text { des surfaces } \\
\text { agricoles totales }\end{array}$ \\
\hline HNM : 0,5 m + 1 m surcote & 10 & 25 & 0,5 \\
HNM : 1 m + 1 m surcote & 12 & 30 & 0,7 \\
HNM : 2 m + 1 m surcote & 20 & 50 & 1,1
\end{tabular}

Les régions du monde dont les terres agricoles littorales sont les plus exposées sont l'Asie du Sud, l'Asie de l'Est et le Pacifique, l'Afrique de l'Ouest et l'Amérique du Sud, où plus de $10 \%$ des terres agricoles littorales sont exposées à une submersion marine. Certains pays pourraient voir leur surface agricole totale se réduire jusqu'à $24 \%$. Ainsi, le Bangladesh, l'Égypte, le Japon, le Myanmar et le Vietnam pourraient subir des pertes 
de terres agricoles supérieures à $10 \%$ (voir Figure 6 ; Chen et al., 2012). La hausse du niveau de la mer pourrait avoir un impact important sur l'agriculture dans les zones de deltas comme celle du Mékong au Vietnam. En Égypte, un accroissement du niveau de la mer d'1 m inonderait $25 \%$ du delta du Nil et $13 \%$ des terres agricoles (Dasgupta et al., 2009). Les terres agricoles d’Indonésie et de Thaïlande seraient également affectées avec une baisse en conséquence de la production de riz.

Figure 6. Impact potentiel de la hausse du niveau de la mer sur les terres agricoles inondées (en \% de terres inondées par rapport à la surface agricole totale du pays) (Chen et al., 2012)

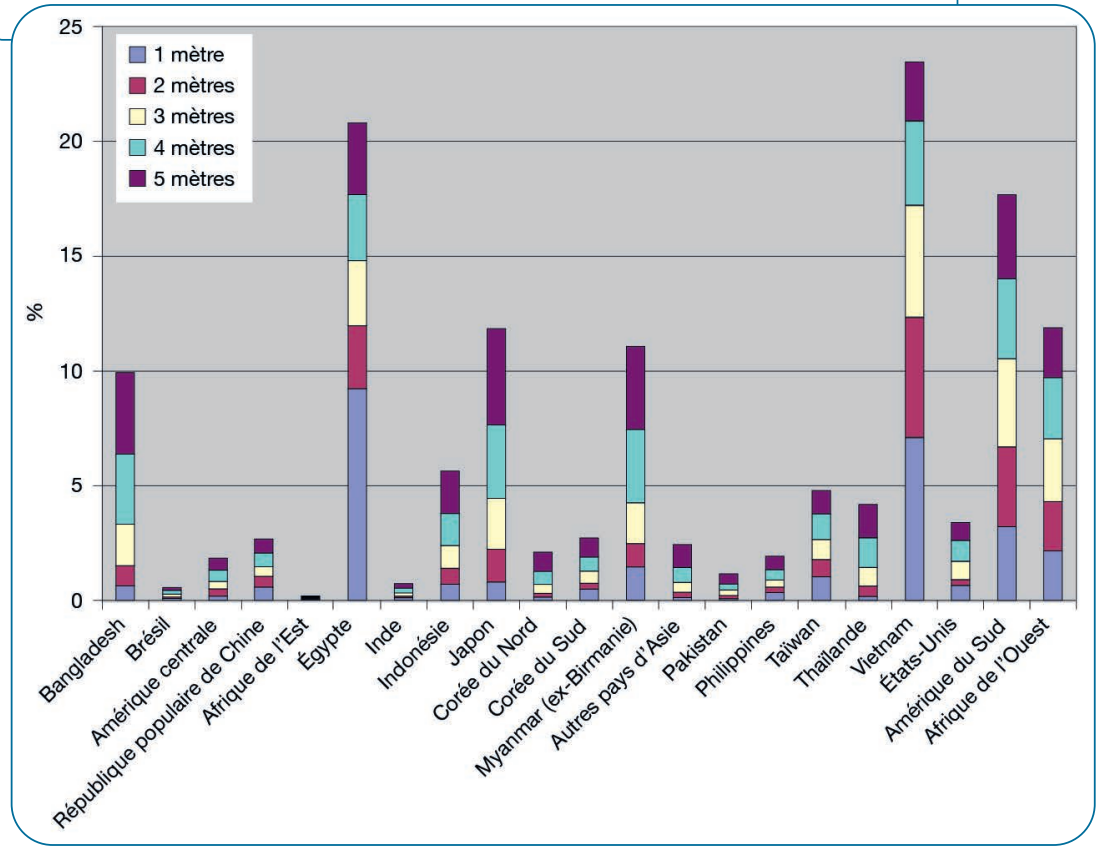

Pour estimer les surfaces futures des terres agricoles en zones littorales, il faut également prendre en compte la diminution des terres agricoles liée à l'urbanisation. Selon Bren D’Amour et al. (2016), 27 à 35 millions d'hectares de terres agricoles pourraient être urbanisés d'ici 2030, dont la moitié en Asie et le reste en Afrique et en Amérique. Plus de $50 \%$ de cette urbanisation globale pourrait avoir lieu dans les zones littorales où sont localisées les mégapoles (Gange-Brahmapoutre, Perle, Jangtze, Bohai, Hokkaïdo, Java, Washington-NY-Boston, Accra-Lagos, delta du Nil). Cela pourrait affecter non seulement des zones de productions de riz en Asie, mais aussi des zones de production de maïs et de blé. 
La réduction des terres agricoles sur les littoraux pourrait engendrer différents types de compensation et de substitution pour garantir l'approvisionnement alimentaire des zones littorales :

- un repli de l'agriculture vers l'arrière-pays lorsqu'il reste des surfaces disponibles potentiellement cultivables, à l'échelle nationale ;

- l'accélération des processus d'accaparement des terres (land grabbing) dans d'autres régions du monde où des terres sont accessibles ;

- une dépendance accrue des zones littorales au commerce international, avec un recours à l'importation de produits agricoles via les marchés internationaux pour assurer l'approvisionnement alimentaire.

\section{Con séquences potentielles sur la place de la pêche et de l'aquaculture}

LES PRODUITS DE LA PÊCHE ET DE L'AQUACULTURE constituent en moyenne 15 à $20 \%$ des apports en protéines et $2 \%$ des apports en calories. Cependant, pour certains pays (comme le Bangladesh ou le Ghana), 50 \% de l'apport protéique est issu des produits aquatiques. On observe une croissance rapide de la demande, qui est passée dans les quarante dernières années de 14 à $20 \mathrm{~kg}$ par habitant et par an. Ces évolutions sont liées à plusieurs facteurs, principalement le changement des styles de vie lié à l'urbanisation, la transition nutritionnelle et le développement de classes moyennes dans les pays émergents (Chine, Inde, pays de la zone ASEAN...).

Les activités de pêche et d'aquaculture sont également des sources d'emploi et de revenus pour les populations littorales, en particulier pour les ménages pauvres. Ainsi au Bangladesh, en Tanzanie ou en Thaillande, plus de la moitié des ménages en zone littorale dépendent à titre principal ou secondaire d'activités de pêches et d'aquaculture (Barbier, 2015).

En termes de production, on observe une stagnation des pêches depuis quinze ans à environ $100 \mathrm{Mt} / \mathrm{an}$. Cela est lié d'abord à la surexploitation qui impacte les trois quarts des stocks existants, à une érosion de la biodiversité marine, à une dégradation des habitats, à une pollution accrue (notamment par les plastiques), et globalement, à une réduction de la résilience des écosystèmes marins. On observe ainsi depuis une trentaine d'années une substitution progressive des débarquements de la pêche par les productions d'aquaculture. Celle-ci continue à se développer rapidement, quoiqu'avec un certain ralentissement dans plusieurs filières comme la crevette ou le saumon. Sa production a dépassé les $70 \mathrm{Mt} / \mathrm{an}$. L'aquaculture est en forte progression dans les zones deltaïques (production de tilapia, mulet, crevettes) et dans les élevages en cage au large (salmonidés, bar, daurade...). Les principaux enjeux du développement de l'aquaculture sont, d'une part de réduire sa dépendance à l'usage de farines et huiles animales (en 
privilégiant notamment la culture d'espèces herbivores), et d'autre part de réduire son impact sur les écosystèmes (FAO, 2020).

Le contexte d'évolution de long terme de ce secteur se caractérise par :

- une dégradation des écosystèmes littoraux tels que les mangroves et les récifs coralliens (qui représentent 20 à $25 \%$ des prises dans les pays insulaires en développement), du fait du changement climatique (hausse des températures, acidification) et de la montée du niveau de la mer ;

- un impact croissant des pollutions marines sur les écosystèmes côtiers ;

- une surexploitation des ressources, liée à la surpêche ; un développement de l'aquaculture avec des élevages d'espèces de poissons plus résilientes à l'acidification et en bas de la chaîne trophique, permettant de se passer de farines animales, et avec des impacts réduits sur les milieux. L'aquaculture pourrait tirer bénéfice de la salinisation des terres basses pour se développer sur d'anciennes terres agricoles, comme c'est déjà le cas dans le delta du Nil en Égypte.

\section{Conséquences potentielles sur la sécurité alimentaire}

LA SOUS-NUTRITION EST UN PHÉNOMÈNE PERSISTANT qui touche 850 millions d'individus, tandis qu'on observe plus récemment une explosion de la malnutrition avec deux milliards d'individus en surpoids et obèses, et une croissance des maladies liées à l'alimentation (diabète, maladies cardio-vasculaires, certains cancers) (Mora, 2018). De plus, les difficultés d'accès des ménages à l'alimentation, du fait de la faiblesse de leurs ressources financières, restent une cause majeure d’insécurité alimentaire dans le monde.

La sécurité alimentaire est un phénomène complexe qui dépend de quatre facteurs : la disponibilité alimentaire, l'accès à l'alimentation des populations, la stabilité de cet accès dans le temps, la nutrition et l'usage des aliments. Pour analyser comment la sécurité alimentaire pourrait être impactée par la hausse du niveau de la mer, nous allons examiner dans quelle mesure les quatre facteurs qui la définissent pourraient être affectés.

Dans une région donnée, la disponibilité alimentaire résulte à la fois des terres cultivées, des rendements des systèmes agricoles, des pertes et gaspillages et des produits importés. Sous l'effet de la hausse du niveau de la mer, la disponibilité en terres par habitant devrait se réduire (augmentation de la population et diminution des surfaces arables) dans un grand nombre de régions. Les régions dont les littoraux sont affectés par l'élévation du niveau de la mer et qui ont peu de réserves de terres cultivables, comme l'Afrique du Nord et le Moyen-Orient, risquent d'accroître leur dépendance vis-à-vis du commerce international pour leur approvisionnement alimentaire (Le Mouël et al., 2017). Le maintien de la disponibilité alimentaire dans les zones littorales dépendra également de la capacité des systèmes agricoles à sécuriser leur production dans un contexte de forte instabilité climatique et de dégradation des sols. Si rien n'est fait, les rendements agricoles pourraient se réduire dans de nombreuses régions. Dans ces conditions, il s'agira 
de favoriser l'adaptation des espèces cultivées, de réduire le recours à l'irrigation et de développer des systèmes agricoles plus résilients, c'est-à-dire des systèmes plus autonomes, s'appuyant sur des rotations longues incluant des légumineuses, développant une complémentarité entre culture et élevage pour boucler le cycle de l'azote, et mettant en pratique des techniques d'agroécologie (protection des sols contre l'érosion, haies, renforcement des structures paysagères).

L'accès à l'alimentation pourrait être affecté par la hausse du niveau de la mer de plusieurs manières. En effet, dans les pays en développement, les activités d'agriculture, de pêche et d'aquaculture contribuent à $50 \%$, voire à $70 \%$, des revenus des ménages pauvres localisés dans les zones littorales. Or ces ménages seront particulièrement vulnérables à la croissance des risques littoraux liés au changement climatique, que ce soit directement par leurs effets sur les conditions de vie, ou indirectement parce que ces changements affectent les écosystèmes littoraux dont ils dépendent (Barbier, 2015). Tout d'abord, les ménages situés sur les littoraux seront affectés directement par des catastrophes naturelles liées à des inondations ou des submersions marines qui risquent de générer des dommages sur leurs propriétés, leurs activités d'élevage ou agricoles, et de réduire leurs revenus tout en accroissant leur vulnérabilité. Or, on sait qu'en zone littorale, les ménages pauvres dépendent pour leur protection de manière exclusive des barrières naturelles, comme les mangroves; et ces zones naturelles sont fréquemment surexploitées et risquent d'être négativement affectées par le changement climatique. Indirectement, les ménages situés sur les littoraux vont subir les effets à long terme du changement climatique et de la hausse du niveau de la mer sur les milieux (salinisation et érosion). Par exemple, les effets de l'intrusion saline sur l'agriculture ont été documentés dans une région littorale au sud-ouest du Bangladesh. En 10 ans, $70 \%$ des agriculteurs ont partiellement ou totalement abandonné l'agriculture du fait de la salinisation des sols et ont, soit converti leurs terres en élevage de crevettes, soit vendu leurs terres ; 75 \% des ménages ont également arrêté l'élevage du fait de l'absence d'alimentation animale, la production de fourrage étant elle aussi impactée par les processus de salinisation. Il faut noter que cette salinisation des terres basses de certains deltas peut donner lieu au développement d'aquaculture d'eau saumâtre même avec des moyens rudimentaires : prélèvement d'alevins dans le milieu naturel et ensemencement dans d'anciennes rizières salinisées, alimentation avec des sous-produits agricoles, polyculture extensive d'espèces, marchés locaux de proximité (Hereher, 2010). Avec ces principes, l'aquaculture égyptienne est ainsi passée de 100 ooo tonnes de production aquacole dans les années 1990 à plus d'un million de tonnes à partir de 2010 (Kara et al., 2016). Consommée localement, cette production a conduit au doublement de la consommation des produits aquatiques en 30 ans soit un passage de 9 à $20 \mathrm{~kg} /$ tête/an (ce qui correspond à la moyenne mondiale).

Par ailleurs, les revenus des ménages pauvres liés à l'agriculture sont durablement réduits par des événements ponctuels comme des submersions marines à l'occasion de cyclones par exemple. Les activités de pêche des ménages pauvres en zone littorale 
sont également affectées par le changement climatique de deux manières : d'une part, les changements physiques dans les écosystèmes marins (température, acidité, circulation marine) vont modifier la structure des communautés biotiques et donc les zones de pêche ; d'autre part, les changements climatiques vont altérer certains écosystèmes littoraux tels que les zones humides, les mangroves, les récifs coralliens, qui sont des habitats vitaux pour la reproduction des espèces. Ainsi, les ressources et les revenus des ménages littoraux risquent d'être négativement impactés par le changement climatique et la hausse du niveau de la mer.

L'accès à l'alimentation est rendu instable par la survenance d'inondations à répétition ou d'événements extrêmes, occasionnant :

- des dégâts ponctuels sur les infrastructures logistiques nécessaires au transport et à la distribution, mettant en péril l'approvisionnement alimentaire ;

- des destructions de cultures, de bétail, des infrastructures aquacoles qui peuvent avoir un effet local sur la production alimentaire et sur les revenus des populations dépendant de ces activités ;

- une réduction de l'apport alimentaire en produits frais, en particulier en fruits et légumes (qui dépendent d'un approvisionnement de proximité) ;

- une dégradation de la qualité et de la potabilité de l'eau.

Enfin, les usages alimentaires pourraient être transformés à la suite de catastrophes climatiques. Les crises et l'aide alimentaire peuvent engendrer une substitution de la consommation de produits traditionnels par des céréales importées, et une modification des régimes alimentaires à long terme (vers des régimes déséquilibrés, voire carencés). De manière générale, il y a un risque important que les impacts du changement climatique et de la montée du niveau de la mer sur les littoraux renforcent un « piège de la pauvreté " pour les ménages pauvres des zones littorales en leur infligeant des pertes économiques directes et en dégradant les écosystèmes littoraux ou marins dont ils dépendent, générant des situations chroniques d'insécurité alimentaire (Barbier, 2015). 


\section{Pour les populations et les villes littorales}

\section{Une littoralisation des populations à l'échelle mondiale}

SUR LES TROIS DERNIÈRES DÉCENNIES, la population littorale (vivant à moins de $100 \mathrm{~km}$ de la côte) est passée de 1,6 milliard en 1975 à 2,5 milliards en 2005, dont 1,8 milliard vit dans les pays en développement (Barbier, 2015). Ce phénomène témoigne de l'attractivité des zones côtières qui repose sur de multiples facteurs : des ressources abondantes, en tant que lieux d'interface terre-mer, et le développement des activités économiques qui s'y concentrent du fait des réseaux de transport et du commerce international. Aussi, les zones littorales connaissent d'importants changements socio-économiques et environnementaux, se traduisant par une urbanisation rapide, une artificialisation des sols, une utilisation massive des ressources et des effets négatifs des activités humaines sur les écosystèmes côtiers. Ainsi, alors que les mangroves, les marais, et les récifs coralliens sont des protections naturelles contre les vagues associées aux tempêtes, ils sont dégradés ou détruits par la croissance démographique et l'urbanisation.

Les populations les plus exposées au risque de submersion sont concentrées dans les zones côtières basses ("Low Elevation Coastal Zone ») qui ont une altitude inférieure à 10 mètres au-dessus du niveau de la mer. Les zones côtières basses correspondent à $2,3 \%$ des terres émergées, la population qui y réside croit de manière régulière depuis trente ans. On estime que 528 millions de personnes y vivaient en 1990, 625 millions de personnes en 2000, 704 millions en 2010. La moitié de la population dans les zones côtières basses est des urbains. À l'échelle mondiale, la population résidant dans les zones côtières basses se concentre principalement dans les pays en voie de développement qui totalisent 578 millions de personnes (ibid.). La plupart des pays ayant des zones côtières basses sont des petites îles, mais aussi des zones de delta densément peuplées. L'Asie concentre les trois quarts de la population totale des zones côtières et les deux tiers de la population urbaine des zones côtières basses (voir Figure 7). La concentration de la population dans les zones côtières basses est aussi forte en Afrique : 7 \% de la population totale et $12 \%$ de la population urbaine sont situées sur $1 \%$ du territoire (McGranahan et al., 2007). 
Figure 7. Population mondiale en 2000 et projection de population à 2025 dans les zones côtières basses.

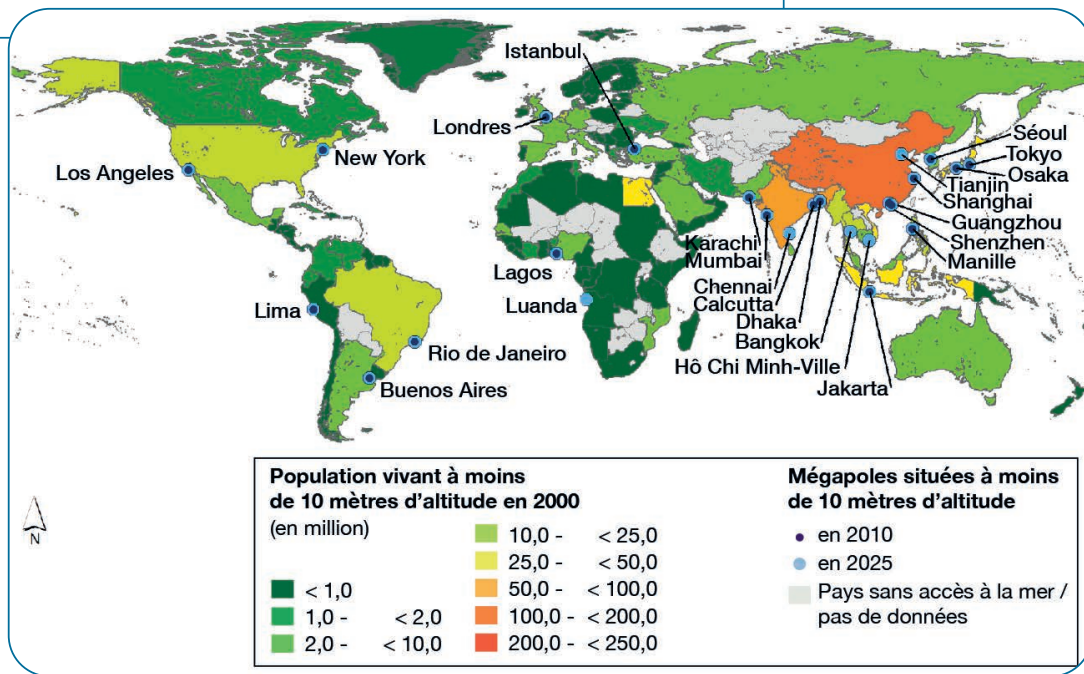

Source : Neumann et al., 2015

\section{Une estimation de la population exposée à une hausse du niveau de la mer en 2100}

Du fait des dynamiques d'évolution de la population littorale et de l'attractivité des zones côtières, en particulier dans les pays en développement, la population dans les zones côtières basses pourrait atteindre selon les projections réalisées par Neumann et al. (2015), 900 millions en 2030 (880-950 millions) et dépasser le milliard d'habitants en 2060 (1-1,4 milliard). La part de la population mondiale dans les zones côtières basses devrait rester stable à $10 \%$ tout au long de la période. Les travaux réalisés par Barbier (2015) estiment qu'en 2100 sur le 1,06 milliard vivant dans les zones côtières, 583 millions résideraient dans des zones urbaines.

C'est dans les pays en développement que la population des zones littorales pourrait croître la plus fortement. À l'horizon 2100, la population des zones côtières pourrait être multipliée par trois ou par quatre suivant les scénarios en Afrique, par deux ou par trois en Océanie, et presque doubler en Amérique latine, tandis qu'elle resterait stable ou légèrement croissante en Europe (Merkens et al., 2016). Cinq pays d'Asie compteront pour la moitié de la population des zones côtières basses : la Chine, l'Inde, le Bangladesh, l'Indonésie et le Vietnam. On estime qu'environ $30 \%$ de cette population pourraient être exposée au risque d'inondation lors d'une onde de tempête centennale (+1 $\mathrm{m})$, soit plus de 300 millions de personnes exposées après 2060 (Neumann et al., 2015). 
Ainsi, selon les projections existantes, la population exposée au risque de submersion devrait augmenter fortement d'ici 2100. Cependant, une forte incertitude reste attachée à ces projections, qui varient beaucoup d'un scénario à l'autre. La trajectoire de développement socio-économique est ici déterminante, car elle structure d'un côté la dynamique de la montée de la mer via les effets des activités anthropiques sur le changement climatique, et conditionne d'un autre côté la capacité des gouvernements et des acteurs à réagir à une hausse du niveau de la mer par des actions appropriées (mais coûteuses après 2050), comme le repli stratégique de populations littorales sur l'arrière-pays.

\section{L'enjeu des migrations et des inégalités liées à une hausse du niveau de la mer en 2100}

La hausse du niveau des mers est déjà une réalité dans certaines zones comme dans les États insulaires, où elle entraîne un processus de déplacement voire de relocalisation des populations. Ces petits États insulaires sont aussi déjà confrontés au problème de la salinisation des terres, avant même les risques de submersion (IPPC, 2014). Ce phénomène est également apparu en Afrique subsaharienne, par exemple au Bénin, où un recul significatif du trait de côte est déjà observable. La salinisation et le recul du trait de côte ont pour conséquence la réduction des terres arables, et donc une réduction des ressources disponibles pour les populations vivant de l'agriculture, ce qui peut générer un phénomène de migration interne. Cependant, la migration est un phénomène complexe et multi-causal. On sait, par exemple, que la propension à la migration est fortement corrélée à un niveau de vie élevé.

Le déplacement des populations engage des enjeux sociaux, culturels et politiques. L'espace, la population et l'organisation politique sont trois strates d'un système et toute migration liée à un changement de l'espace bouleverse leurs interfaces. On distingue en théorie les migrants environnementaux, économiques ou politiques, mais en réalité les facteurs déclenchant sont multiples et s'entremêlent, même si leurs pondérations respectives varient selon les populations, les cultures et l'ampleur des contraintes. Le déplacement des populations engage également une remise en cause du lien entre État, territoire et souveraineté. La Nouvelle-Zélande a choisi de créer un statut de réfugié climatique (visa), mais des difficultés de relocalisation des populations subsistent, en raison du lien culturel fort entre les habitants et leur île. Même si un désir d'Occident se fait ressentir chez les jeunes, il s'agit alors d'une stratégie choisie d'éducation pour préparer à l'exil. Selon les statistiques de migrations intra-étatiques, 25 millions de personnes sont déplacées chaque année par des événements climatiques extrêmes - soit trois fois plus que par les conflits - dont $86 \%$ suite à des catastrophes naturelles hydro-climatiques, l'Asie étant le continent le plus concerné (Kalin, 2014).

À l'horizon 2100, le changement climatique et la hausse du niveau de la mer vont diminuer les ressources des populations les plus défavorisées. Cependant, alors que certaines politiques environnementales envisagent la migration comme une manière pour 
les populations de s'adapter aux effets du changement climatique, de manière générale, les politiques migratoires des États visent a contrario à limiter les migrations (Gemenne et Blocher, 2017). Cela laisse présager de fortes tensions d'ici à 2050 sur les questions migratoires et plus encore après 2050 avec l'accélération possible de la hausse du niveau de la mer. Aussi, la création d'un statut de « réfugié climatique » est une voie envisagée pour accompagner les migrations internationales face au changement climatique (ibid.). Dans le cas contraire, de fortes migrations internes devront être gérées par les États. Mais ce statut, qui devrait être logiquement géré par les Nations Unies via le Haut-Commissariat aux réfugiés, pose des problèmes juridiques, économiques, culturels et géopolitiques complexes et de grande ampleur.

\section{Con séquences potentielles sur les zones urbaines littorales}

LA LOCALISATION DE LA POPULATION URBAine dans les zones littorales est un phénomène ancien et qui perdure. Depuis l'époque antique ou médiévale, les populations se sont concentrées dans des villes portuaires, aussi bien en Extrême-Orient que dans le bassin méditerranéen ou en mer du Nord. À l'époque coloniale, les villes littorales se sont développées par l'accroissement du commerce maritime. La plupart des métropoles littorales mondiales, comme Londres, New York et Tokyo doivent leur puissance au commerce maritime passé (Béchet et al., 2017). Sur les six plus grandes villes du monde, trois sont des méga villes littorales : Tokyo, la plus grande agglomération du monde (38 millions d'habitants), Shanghai avec 23 millions d'habitants et Mumbai avec 20 millions (WUP, 2018). Dans $60 \%$ des pays ayant une façade maritime, les principales villes sont situées dans la zone littorale et comptent pour 70 \% de la population urbaine (Noin, 1999).

L'étude menée par Kummu et al. (2016) à l'échelle mondiale montre qu'en 2010, sur une bande littorale étendue (définie comme la zone à moins de $100 \mathrm{~km}$ des côtes et à moins de 100 m d'altitude) qui s'étend sur 12,3 millions de $\mathrm{km}^{2}$ et recouvre $9 \%$ de la surface totale émergée, $63 \%$ de la population vit dans des villes (contre $51 \%$ à l'échelle mondiale). Cette bande littorale concentre également les activités économiques puisque $42 \%$ du PIB mondial y est produit. Il y a cependant des différences importantes d'urbanisation et de densité de population au sein de la bande littorale. À proximité de la côte (à moins de 20 km des côtes et à moins de 20 m d'altitude), le niveau d'urbanisation est de $69 \%$, soit quatorze points au-dessus de la moyenne mondiale. Dans l'arrière-pays (à plus de $20 \mathrm{~km}$ et à moins de $100 \mathrm{~km}$ des côtes), le niveau d'urbanisation, de $59 \%$, reste plus élevé que la moyenne mondiale. À l'échelle globale, les villes se concentrent sur la bande littorale à proximité des côtes. Ainsi, 17 des 30 plus grandes villes du monde (ayant une population supérieure à 5 millions d'habitants), sont localisées à moins de 20 km des côtes. Plus d'un tiers des villes de plus d'un million d'habitants (88 sur 226) sont également localisées sur la bande littorale. 
Or, d'ici 2100, la population urbaine et l'urbanisation littorales vont s'accroître. La population urbaine de la bande littorale « étendue » pourrait atteindre 1,9 milliard d'urbains, soit $80 \%$ de la population littorale (urbains et ruraux) dès 2050. Ainsi, le niveau d'urbanisation sur la bande littorale resterait très supérieur à la moyenne mondiale qui atteindrait $66 \%$ en 2050 selon les projections du WUP (2018).

Les populations urbaines localisées dans les zones littorales sont particulièrement exposées aux risques liés à l'élévation du niveau des mers, aux tempêtes, aux inondations et à la submersion marine. D'autant plus que, dans les zones urbaines littorales, la dégradation des écosystèmes littoraux, l'artificialisation des sols et les phénomènes de subsidence des sols, en lien avec l'expansion urbaine et les activités agricoles, diminuent la protection des littoraux contre les désastres naturels et accentuent les effets à long terme du changement climatique en favorisant l'intrusion saline et l'érosion des sols.

\section{I'exposition des villes au risque de submersion}

Dans l'hypothèse d'une élévation d'un mètre du niveau de la mer à 2100, accompagnée par une intensification de $10 \%$ des ondes de tempêtes, l'étendue des zones littorales urbanisées exposées au risque passerait en moyenne de $7 \%$ à $13 \%$ du total des espaces urbains littoraux (Dasgupta et al. 2011). Les populations urbaines les plus exposées sont principalement localisées en Asie, dans les pays en développement (Indonésie, Philippines, Vietnam...), ainsi qu'aux États-Unis, au Japon et aux Pays-Bas. Deux pays d'Afrique figurent parmi les quinze pays les plus exposés, l’Égypte et le Nigeria, et un seul pays d’Amérique latine, le Brésil. En termes de surfaces urbaines affectées, la région Moyen-Orient et Afrique du Nord serait particulièrement touchée avec en moyenne $20 \%$ des espaces urbains littoraux exposés aux risques (Tunisie $31 \%$, Yémen $39 \%$, Arabie Saoudite $26 \%$ ) (ibid.).

Brecht et al. (2012) estiment que $67 \%$ des populations urbaines littorales exposées à un risque centennal de submersion marine se concentrent dans 10 villes. Celles-ci sont situées dans les pays en développement, principalement en Asie (Chine, Inde, Bangladesh, Japon, Indonésie, Thaïlande, Vietnam). Le World Urbanization Prospect (WUP, 2018) estime, qu'en 2030, il y aura 43 méga villes (ayant plus de 10 millions d'habitants), alors qu'il n'y en avait que 28 en 2014, et que plus de la moitié de ces méga villes seront localisées dans les zones littorales (voir fig. 7).

En 2070, la population exposée au risque d'inondation et de submersion marine dans les grandes villes pourrait être multipliée par trois, du fait des effets combinés de l'élévation du niveau de la mer, de la subsidence, de la croissance de la population et de l'urbanisation (Hanson et al., 2011). L'exposition au risque se concentre sur un petit nombre de villes. Ainsi, en 2070, 15 des 20 villes en termes de population les plus exposées au risque de submersion seront en Asie (ibid.). Clark et al. (2016) estime qu'en 2070, 25 méga villes auront plus de $50 \%$ de leur population potentiellement affectée par des phénomènes de submersion marine. 
En termes économiques, l'étude sur l'exposition au risque des villes portuaires mondiales - de plus d'un million d'habitants - montre que les villes les plus exposées aujourd'hui à un risque centennal de submersion marine se situent aux États-Unis, au Japon et aux Pays-Bas (Hanson et al., 2011). Si l'on prend en compte les dynamiques d'urbanisation, il apparaît que les 20 villes les plus vulnérables à l'horizon 2050 à des pertes économiques sont majoritairement dans les pays en développement (Hallegatte et al., 2013). Seulement trois villes sur les vingt seront dans les pays développés : Miami, La Nouvelle-Orléans et Boston. Les pertes globales liées au phénomène de submersion marine, estimées à 6 milliards de dollars par an en 2005, seraient multipliées par 10 en 2050, sous les effets combinés de mécanismes de subsidence et d'élévation du niveau de la mer (ibid.). Compte tenu du rôle économique majeur des villes dans l'économie mondiale, les effets de long terme de l'élévation du niveau de la mer sur les activités économiques seront massifs.

\section{Les infrastructures littorales et les enjeux de résilience}

Les infrastructures littorales (transport, énergie, communications, approvisionnement en eau potable, services, banques...) sont sensibles aux submersions marines et aux inondations. Ces effets vont s'intensifier en conséquence de l'élévation du niveau de la mer.

Les infrastructures littorales seront affectées de multiples manières par les événements extrêmes : dégradation accélérée des réseaux, exposition croissante des infrastructures à des événements climatiques extrêmes, dysfonctionnements en cascade en cas de catastrophe, du fait de l'interdépendance croissante des réseaux notamment électriques. Des catastrophes naturelles peuvent engendrer la défaillance ou la destruction d'infrastructures critiques, immobilisant le système de transport, provoquant des coupures d'électricité, et engendrant une contamination par l'eau de mer de l'approvisionnement en eau potable. Une étude récente sur l'impact de la hausse du niveau de la mer sur les infrastructures au Royaume-Uni montre ainsi que l'ensemble des infrastructures seraient impactées par une submersion marine d'ampleur : transport ferroviaire, routier et maritime, approvisionnement en eau potable, installations sanitaires et réseau d'assainissement, centrales électriques et réseaux électriques de distribution (Dawson et al., 2015).

Azevedo et Mostafavi (2016) identifient quatre enjeux pour accroître la résilience des infrastructures. Le premier concerne la capacité des acteurs à investir pour la maintenance, la réhabilitation et l'adaptation des infrastructures face à la hausse du niveau de la mer. Un second enjeu a trait à l'évaluation et l'anticipation des risques, et à la collecte des informations permettant d'évaluer de façon fiable et pérenne la situation (par exemple, concernant la subsidence d'infrastructures côtières ou de remblais). Le troisième enjeu est le nécessaire engagement des communautés locales et éducatives pour développer une culture et une mémoire du risque, afin de réduire les vulnérabilités et d'agir de manière adéquate. Le quatrième enjeu consiste à réduire les coûts et les risques de la mal-adaptation. Il s'agit de faire en sorte que les investissements dans les infrastructures soient faits au bon moment, qu'ils ne soient pas mal dimensionnés, et qu'ils ne deviennent pas trop rapidement obsolètes sous l'effet de l'accroissement du niveau de la mer. 


\section{Les stratégies d'adaptation et de protection des zones urbaines littorales}

Les STRATÉgies D'ADAPTATION DES ZONES URbaines littorales face aux risques de submersion peuvent schématiquement se diviser d'une part, en des stratégies d'intervention pour la protection des littoraux et d'autre part, en des mesures plus générales de gestion des zones littorales.

Une première catégorie de mesures de protection des zones urbaines littorales concerne la construction de digues côtières, d'épis, de polders, de barrières fixes contre les vagues. Une seconde catégorie de mesures vise à développer des «zones tampons » en renforçant la résilience des écosystèmes littoraux et côtiers. Il s'agit alors de restaurer à grande échelle des zones humides du littoral, de renforcer et de replanter des mangroves, de protéger des récifs coralliens ou de renforcer un cordon dunaire. De telles stratégies peuvent également consister à libérer de l'espace pour les processus sédimentaires et s'affranchir de la nécessité de mener des interventions.

Les mesures de gestion des zones littorales regroupent entre autres des actions contre la subsidence, des règlementations urbaines, des mesures de relocalisation et des politiques d'aménagement de l'espace. Les mesures à mettre en œuvre pour freiner la subsidence, particulièrement importante dans les zones de delta, incluent la réduction du dragage et des prélèvements de sables, le maintien des apports des sédiments dans les zones aval - en limitant par exemple les barrages construits en amont - et la régulation des prélèvements d'eau pour l'irrigation. Un autre type d'action vise à réduire le coût et l'impact des tempêtes sur les littoraux en développant des systèmes d'anticipation d'alerte précoce et des systèmes d'évacuation. D'autres actions consistent à développer ou à réformer des systèmes d'assurance et d'indemnisation des dommages aux biens, tout en palliant les fortes inégalités face aux événements météorologiques extrêmes (cyclone de type Katrina, par exemple). Il semble nécessaire de repenser les principes et les règles de fonctionnement de ces dispositifs assuranciels face à l'accroissement des dégâts et des dommages liés au changement climatique. D’autres actions s'appuient sur le code de la construction, afin de le rendre plus restrictif, et sur la transformation des bâtiments afin qu'ils soient adaptés au risque d'inondation.

L'adaptation des zones urbaines littorales peut également passer par une relocalisation des activités et/ou des populations. On parle alors d'une stratégie de retrait. Elle consiste à retirer les activités et les habitats les plus exposés sur le trait de côte pour les relocaliser dans des zones non exposées à moyen ou long terme. Ainsi, les instruments de planification urbaine peuvent être mobilisés pour réduire l'exposition au risque en zone intra-urbaine et à une échelle territoriale plus large, il s'agit de mettre en œuvre un aménagement intégré des espaces urbains littoraux et ruraux, dans une perspective de recomposition territoriale face aux risques liés à l'élévation du niveau de la mer.

Il apparaît là une tension entre les stratégies d'adaptation des zones urbaines littorales qui peuvent conduire à des formes de mal-adaptation. Comparant différentes catastrophes 
récentes en s'intéressant aux « trajectoires de vulnérabilité », Magnan et Duvat (2015) identifient le mythe du " développement sûr », « maîtrisé » qui accroît en fait la vulnérabilité des sociétés aux aléas naturels. Ce mythe correspond à une confiance trop importante dans une culture d'ingénierie promouvant des solutions techniques lourdes comme les digues ou les barrages. Cette croyance en la maîtrise technique va de pair avec une perte de « conscience du risque » qui peut conduire à un déni des contraintes environnementales. Il s'ensuit un affaiblissement progressif de la « mémoire » du risque et de l'anticipation du risque sur le territoire. Ainsi, l'occupation humaine de zones «à risque " se développe, ce qui augmente l'exposition des populations et engendre une dégradation des écosystèmes naturels «tampon » sous l'effet des activités humaines. C'est ainsi qu'une confiance abusive en des dispositifs techniques de protection peut conduire à des catastrophes d'une ampleur bien plus grande que d'autres stratégies d'adaptation (comme des options de retrait du littoral), comme en témoignent les catastrophes engendrées en 2010 en Charente-Maritime en amont de la tempête Xynthia et, en 2005, par le cyclone Katrina à la Nouvelle-Orléans.

De manière générale, l'adaptation des littoraux urbanisés nécessitera des investissements importants, publics et privés, et une forte capacité d'intervention des administrations locales et nationales. Cela implique le développement de partenariats locaux ou nationaux et des formes élargies de gouvernement urbain. La définition et la mise en place des mesures d'adaptation demanderont par ailleurs d'impliquer les populations locales dans ces dispositifs afin d'accroître la compréhension commune des enjeux et des risques associés pour implémenter des politiques publiques sur plusieurs années, voire sur des décennies. 


\section{Pour la vie économique}

\section{Un enjeu complexe et multiforme}

L'OCÉAN ET SON LITTORAL SONT SOUVENT présentés comme de nouvelles et prometteuses frontières économiques, riche de ressources, d'innovations et d'emplois. L'économie maritime, parfois confondue avec l'économie « bleue » qui, elle, respecte les principes de durabilité et donc exclut notamment l'exploitation des ressources énergétiques fossiles, a de multiples définitions selon les pays. L'usage le plus courant est celui des activités ayant un lien direct avec la mer (ce qui disparaîtrait si la mer n'était plus là), selon une conception traditionnelle de ces secteurs : pêche, aquaculture, transport maritime, activités portuaires, tourisme littoral, biotechnologies, mais aussi ressources naturelles minérales et énergétiques (fossiles et renouvelables) et services fournis par les écosystèmes océaniques (fixation de $\mathrm{CO}_{2}$, émission d'oxygène...). L'océan joue donc un rôle croissant dans de nombreux domaines vitaux pour la sécurité économique et environnementale du monde comme l'alimentation, l'énergie ou la régulation de grands équilibres de l'environnement. L'OCDE a estimé en 2010 le poids de ce domaine à 1500 milliards \$, soit 2,5\% de la valeur ajoutée mondiale (GVA). Une récente étude européenne sur l'économie maritime donne le chiffre de 2500 milliards \$, soit l'équivalent de la $7^{\mathrm{e}}$ puissance économique du monde (EC, 2020). Le pétrole et le gaz offshore totalisent un tiers de cette GVA, suivis par le tourisme maritime et côtier (26\%), les ports (13\%), l'équipement maritime (11\%). L'emploi direct représentait environ 31 millions d'ETP en 2010, pour plus du double en 2020. Les secteurs majeurs d'emploi sont, dans l'ordre, les pêches (36\%), puis le tourisme (23\%). Le transport maritime permet d'échanger $90 \%$ des produits mondiaux et constitue un énorme levier de développement à l'échelle mondiale. Les activités de l'économie maritime fournissent un moyen de subsistance à environ 820 millions de personnes.

En France, l'économie maritime représente $14 \%$ du PIB, avec 270 milliards $€ /$ an, soit près de 470000 salariés, 58000 entreprises, 900 métiers (source : Fondation de la mer/BCG). Ce secteur pèserait trois fois le secteur automobile et cinq fois l'aéronautique. Mais son premier moteur est le tourisme, activité vulnérable à bien des crises.

Les projections tendancielles à 2030 donnent toujours de fortes croissances dans la plupart des secteurs, notamment dans l'énergie éolienne marine (+ $24 \%)$, l'aquaculture (plus de la moitié de l'apport en protéines aquatiques, soit $16 \%$ de l'apport protéinique mondial, avec une croissance d'environ $7 \%$ par an sur les 20 dernières années), la transformation des produits de la mer, les biotechnologies marines (+10\%/an) et les ports.

Mais la plupart des études sur l'économie marine ne prennent pas en compte le changement global et notamment la montée du niveau de la mer. Certes, il est difficile de dissocier les impacts spécifiques de cette dernière car elle croise de multiples effets du 
changement global, comme l'augmentation de la puissance des événements météorologiques extrêmes sur les côtes (liée à la hausse de la température de la mer) ou la vulnérabilité accrue des côtes dans le cas de la perte de récifs coralliens (liée à l'acidification de l'océan). Ces effets réduisent la résilience des côtes et les ressources qu'en tirent les populations locales. En matière d'environnement marin, le tableau général est plutôt sombre car les tendances connues sont inquiétantes : réchauffement des eaux superficielles (90\% de la chaleur excédentaire est absorbée par la mer), acidification et désoxygénation entraînant de multiples conséquences sur les écosystèmes, des perturbations dans les chaînes alimentaires, une érosion accrue de la biodiversité marine, des pertes de biomasse... Ces impacts, pour la plupart négatifs, sont encore mal connus, même dans le cas d'une élévation thermique limitée à $2{ }^{\circ} \mathrm{C}$ à 2100 . II persiste un fort déficit en connaissances pour comprendre les dynamiques à l'œuvre, en estimer les impacts de manière plus précise, région par région, et proposer des voies de protection et de prévention adaptées. De plus, toute la pollution des bassins versants finit à la mer, notamment les plastiques, en imprégnant particulièrement les zones côtières. Celles-ci sont d'autant plus vulnérables que nombre de décharges sauvages persistent sur le littoral et que la montée de la mer commence à concerner des décharges terrestres et des friches industrielles non dépolluées après le départ des industries. Mais aucun acteur ne se sent assez responsable pour payer la dépollution des sites exposés à la submersion.

\section{Des approches quantitatives}

Il faut reconnaître d'emblée qu'essayer d'évaluer les coûts d'une submersion progressive des zones côtières dans le monde avec des valeurs de hausse de la mer variant d'un facteur 3 et des contextes démographiques, géopolitiques, économiques et sociaux très divers reste un travail à considérer avec prudence. Seuls les ordres de grandeur peuvent être retenus, car il s'agit d'impacts incommensurables au sens strict. Il est pourtant utile de tenter de le faire, car cela peut constituer un socle de réflexion et de décision pour nombre de décideurs, à l'instar du rapport Stern (2006) sur les impacts du changement climatique sur l'économie mondiale.

L'OCDE a publié en 2019 les résultats d'une étude de plusieurs années sur le coût économique de la montée du niveau de la mer. La base de calcul utilise le modèle Diva qui intègre 12148 segments de côtes répartis dans le monde afin de construire un échantillon représentatif de la situation mondiale (Vafeidis et al., 2008). Le résultat principal est que, si l'on ne fait rien ou très peu en matière d'atténuation du changement climatique, ce qui implique un scénario climatique de type RCP 8.5 (le pire) et une montée de 1,30 m en 2100, le coût des dégâts liés à la submersion et aux événements météo extrêmes sur le littoral aboutirait à environ 50000 milliards \$/an, soit 4 \% du PIB estimé du monde. Ces coûts peuvent être divisés par 100 en cas d'adaptation généralisée et précoce, mais cette hypothèse reste peu probable. Cette adaptation coûterait entre 15 et 70 milliards \$/an selon les scénarios socio-économiques proposés par Hinkel (2014). 
Si l'on se focalise sur une analyse en termes de coûts-bénéfices, il semble alors justifié : - de protéger $13 \%$ des côtes mondiales (= $90 \%$ de la pop. côtière et $96 \%$ des richesses); - de ne rien faire pour $65 \%$ de ces côtes ( $=0,2 \%$ de la pop. côtière et $0,2 \%$ des richesses) ; - d'ajuster par repli stratégique selon les situations pour $22 \%$ (= 9,8\% de la pop. côtière et 3,8\% des richesses).

Le repli stratégique pourrait se révéler la solution la plus économique à long terme pour la majorité des activités humaines en région côtière vulnérable, en dehors des villes importantes et riches où la protection sera longtemps « rentable ». Néanmoins, le retrait sera difficile à financer en raison de la préférence générale de la majorité des acteurs pour le court terme. L'État de Californie (39 millions d'habitants ; $6^{e}$ puissance économique du monde en termes de PIB, devant la France) a fait mener une série d'études par le bureau du planning et de la recherche du gouverneur de l'État de Californie depuis 2006 (2009, 2012, 2018) avec les concours de centaines d'experts de grandes universités (Stanford, Berkeley...) et d'instituts fédéraux spécialisés (Bedsworth et al., 2018). L'étude part de deux hypothèses contrastées du GIEC : une hypothèse basse (RCP de 4.5 correspondant à un taux de $\mathrm{CO}_{2}$ de 550 ppm en 2100) et une hypothèse haute, en tendanciel actuel (RCP de 8.5 correspondant à un taux de $\mathrm{CO}_{2}$ de 900 ppm en 2100). La montée relative du niveau de la mer pourrait atteindre entre $1,80 \mathrm{~m}$, à $3 \mathrm{~m}$ en 2100 , en tenant compte des phénomènes locaux de subsidence qui peuvent atteindre $2 \mathrm{~cm}$ par an, comme dans le delta de Sacramento, débouché de la principale vallée agricole. Or, environ $55 \%$ de la population californienne est vulnérable à partir d'une hausse de 1,50 m. Les 19 comtés littoraux totalisent $22 \%$ de la surface de la Californie, $68 \%$ de la population et $80 \%$ du PIB. Les impacts du changement climatique sur le littoral seraient multiples et croisés via la température et l'acidité de la mer, la hausse de son niveau, les efflorescences d'algues toxiques, la réduction des forêts marines d'algues, fixatrices de $\mathrm{CO}_{2}$, les changements d'écosystèmes marins productifs, les événements météo extrêmes. En conséquence, les coûts économiques associés se chiffrent en centaines de milliards de dollars à l'horizon 2050. Les secteurs le plus touchés sont, dans l'ordre (en milliards de \$) : les propriétés côtières (80-126), la santé (50-85 pour une vie humaine estimée à 7 millions \$), les infrastructures (48), l'effet des inondations dans l'arrière-pays (42), les grandes villes (30).

Les autorités envisagent déjà le repli stratégique de certaines villes. Sur le moyen terme, l'État de Californie prépare un vaste programme d'amélioration de la résilience globale de tous les systèmes impliqués dans les grands domaines vitaux : environnement, agriculture, infrastructures, énergie, villes, eau. Ce programme, Rebuild by Design, a pour objectif de « reconnecter la nature, l'homme et les infrastructures face aux changements à venir ».

\section{La difficile répartition des responsabilités}

DANS TOUTES LeS RÉGIONS CôtIÈres, il n'existe pas de risque zéro. Par ailleurs, le niveau d'acceptabilité des risques par les populations et les décideurs politiques est fonction de nombreuses variables, ce qui implique une approche spécifique des situations. II faut 
rappeler que le risque n'est pas seulement le résultat de la multiplication d'un aléa (probabilité d'occurrence) et d'une vulnérabilité (ampleur des impacts potentiels) selon l'acception courante, car il doit être diminué par le degré de connaissance des deux termes par la population concernée. Ainsi, aux Antilles, en 1979, le cyclone David n'a fait aucun mort en Martinique, contre plus de 50 dans l'île voisine de la Dominique. L'explication tient surtout dans la qualité d’information de la population en temps réel.

En matière de répartition des responsabilités, plusieurs aspects doivent être pris en compte.

\section{Les stratégies de gestion des risques côtiers}

Chaque stratégie présente des avantages et des inconvénients : la protection est souvent justifiée à petite échelle, pour des patrimoines à valeur élevée, mais elle reste coûteuse, avec des effets secondaires comme l'accroissement des risques d'inondation sur les bords. Il faut essayer de favoriser des processus naturels (mangrove, marais...) plutôt qu'artificiels (recharge de sable, par exemple). L'adaptation implique d'anticiper l'urbanisation et l'architecture de toutes les infrastructures et constructions. Elle n'est possible qu'à la condition d'une prise de conscience globale par les citoyens, les politiques et les responsables de la vie économique. Le retrait concerté et anticipé est la meilleure stratégie en termes de coûts sur le long terme, mais son déclenchement dépend souvent de l'expérience préalable de catastrophes par les populations.

\section{La politique économique de l'adaptation côtière}

Même si elle est décidée démocratiquement, toute politique d'adaptation génère des conflits entre les acteurs dans divers secteurs (immobiliers, tourisme, pêche, assurance...). Il faut réussir la mise en cohérence des prises de conscience de la nécessité d'agir, des incitations financières, des compétences et des rôles des acteurs. Les choix issus des interactions entre acteurs dépendent de l'équilibre entre les contributions du public et du privé, des calculs de rentabilité à court ou long terme, et surtout de l'assiette des taxes pour financer les efforts. Est-ce à la commune, à la région ou au pays entier de supporter les coûts de protection ou de repli ? Les touristes souhaitent aussi profiter d'une plage stable.

\section{L'enjeu pour les compagnies d'assurances et de réassurance}

Il s'agit d'assurer les biens contre les risques de submersion et de tempête ni trop tôt, ni trop tard, ni trop cher, ni trop peu cher (Chneiweiss et Bardaji, 2020). En termes d'assurances, avec la montée inexorable du niveau de la mer, on passe progressivement de « la catastrophe exceptionnelle », assurable, à la reconnaissance d'un aléa connaissable, donc prévisible, donc non assurable. La valeur d'un bien immobilier proche de la côte reste plus élevée qu'à l'intérieur des terres (+ 100 \% aux États-Unis), tant que le risque 
d'inondation est considéré comme faible... jusqu'à ce qu'une catastrophe advienne. Celle-ci va alors déclencher des effets de dévalorisation en cascade. Une étude de la caisse de réassurance française de 2018 projette un accroissement des coûts de catastrophes naturelles à 2050 de $23 \%$ pour des sécheresses, de $38 \%$ pour les inondations et de $82 \%$ pour les submersions. Même les États ont une perception variable des pourcentages du coût d'une catastrophe à allouer à la prévention pour éviter une récidive : $3 \%$ au Mexique et $25 \%$ au Japon.

\section{Le rôle de l’État}

L'intégration de la submersion marine dans les outils économiques et de régulation est principalement du ressort de l'État pour diverses fonctions : évaluation des risques liés aux submersions et aux tempêtes, réglementation et normes de construction en zone exposée, développement des politiques de gestion intégrée de la zone côtière, préparation, via la réassurance, de la transition vers des prix plus réalistes en fonction des risques, information obligatoire sur les risques encourus par un bien immobilier près de la mer dans toute transaction (comme en Californie et en Floride). Dans la pratique, peu de pays provisionnent des réserves pour les risques de submersion et il existe rarement d'outils juridiques, réglementaires et financiers pour répondre aux enjeux de la dynamique littorale (Buchou, 2019). La tendance actuelle est d'impliquer les acteurs privés et notamment les entreprises situées en zone exposée. Toutefois, il n'existe pas deux plans d'évaluation et de prévention comparables dans tous les pays de l'OCDE, même s'ils sont tous fondés sur des indicateurs similaires. La valeur de ces plans reste limitée en raison de l'absence de séries temporelles longues de mesures, de l'imprécision des recommandations d'action en cas de menace, et enfin du manque de procédures pratiques pour la mobilisation des financements. L'implication de l'État dépend surtout de sa vision politique de la menace et de ses conséquences financières. Dans les pays de l'OCDE, la prise en compte de la hausse du niveau de la mer concerne :

- à $90 \%$ l'information sur les risques et les impacts potentiels ;

- à $63 \%$ des évaluations qualitatives et quantitatives des impacts potentiels ;

- à $50 \%$ des cadres législatifs et réglementaires spécifiques à ce type de risque ;

- à $20 \%$ des plans de financement prévisionnels ou des provisions ad hoc.

\section{Les politiques d'adaptation côtière}

L'OCDE a proposé six principes en 2015 : (1) impliquer tous les acteurs ; (2) montrer clairement les objectifs ; (3) mettre des moyens pour une information de qualité ; (4) accompagner les acteurs pour un suivi efficace ; (5) construire un cadre légal et réglementaire adapté ; (6) conserver de la flexibilité afin de faciliter l'adaptation du dispositif. L'application de ces principes de gouvernance dépend beaucoup des cadres législatifs des pays et aussi des mentalités par rapport à ce type de danger. Ainsi, en NouvelleZélande, les autorités ont préparé un plan de prévention pour une submersion de 1,50 m 
en 2100. Cette submersion pourrait concerner 133000 personnes, 68 ooo bâtiments et 5 aéroports sur $2100 \mathrm{~km}$ de côtes et un coût estimé de 19 milliards \$. Les cinq points du plan, applicables à tout site côtier, traitent successivement de l'état des lieux, de l'évaluation des enjeux, des options d'action, des applications et enfin du suivi des travaux. Ainsi, la baie de Hawke (94 km de long) est considérée comme vulnérable. Depuis 2018, un comité des acteurs locaux est chargé de proposer une stratégie d'aménagement basée sur la modélisation de la submersion marine et de ses impacts sur l'habitat et les activités humaines dans 16 unités spatiales. Quelques leçons peuvent déjà être tirées. Il faut prendre du temps pour expliquer la situation à tous les acteurs, croiser le politique et le scientifique, donner la responsabilité aux communautés locales et, surtout, préparer tôt les propositions de répartition possible des financements. Avec la montée des risques de catastrophe économique en cas d'impréparation des acteurs, cette leçon locale pourrait bien avoir une portée universelle.

\section{Conclusion}

SI CERTAINS PAYS ONT PARFOIS des plans, chiffrés, de restructuration territoriale avec des analyses coûts-bénéfices, sans que cela implique pour autant leur mise en œuvre, dans beaucoup de pays émergents, il n'existe pas de réflexion à long terme sur le risque économique de submersion. Certains pays vulnérables essayent de préparer des plans, comme le Bangladesh qui s’appuie sur des experts et des modèles de simulation pour des recommandations de prévention, d'évacuation et de reconstruction (Nicholls et al., 2014). Mais faute de solution de long terme, les populations ont tendance à vivre avec le risque plutôt que de partir. Les décideurs raisonnent à 2030, 2050 au maximum, alors que la hausse projetée reste modeste $(+20$ à $30 \mathrm{~cm})$, y compris dans le pire scénario, et leur priorité est toujours à l'économie. Or, il n'est pas facile de démontrer que les coûts seront plus forts si l'adaptation n'est pas rapide et d'envergure et si elle ne s'accompagne pas d'une politique d'atténuation du changement climatique à l'échelle mondiale. "Sur le terrain, les gens voient des digues » disent la plupart les élus pour se rassurer. De fait, comment évaluer les conséquences de moyen et long terme de la salinisation des terres, de la perte des mangroves, de la modification des écosystèmes productifs côtiers, de la baisse du tourisme littoral, de la migration à terme des élites comme des populations pauvres ? L'approche économique est utile, mais pas davantage au nord qu'au sud, elle ne joue un rôle déterminant dans les choix d’aménagement des décideurs. 


\section{Pour la géopolitique}

LA MER A, PAR NATURE, une dimension géopolitique. Les raisons à cela sont multiples : elle borde la majorité des pays du monde, dont presque tous les grands pays; elle est le support de $80 \%$ du commerce mondial, elle recèle des richesses minérales, énergétiques et vivantes considérables et encore mal connues (une bonne partie de celles-ci étant en haute mer sous juridiction internationale), elle offre un espace où peuvent se projeter les grandes puissances militaires, et enfin elle joue un rôle fondamental dans les grands équilibres de la planète. Cette dimension géopolitique de la mer s'exprime dans deux sens en ce qui concerne la montée de son niveau :

- d'abord, cette montée va avoir des impacts croissants sur les enjeux considérés comme géopolitiques au sens courant du terme : territoire, souveraineté, défense, sécurité des ressources, jeux d'alliances, etc.

- ensuite, cette montée est la conséquence d'un système économique fondé sur une exploitation de la nature qui en néglige encore trop les conséquences sur les moyen et long termes. Ainsi, l'activité industrielle en région arctique émet des particules qui vont se poser sur la neige et réduire la réflexion de la lumière solaire, accélérant d'autant la fonte des glaces impactées. Il est donc difficile de dissocier la géopolitique de l'économie, même dans le cadre des enjeux de la hausse du niveau de la mer, car celle-ci est l'une des conséquences de l'accumulation des gaz à effet de serre, eux-mêmes liés à des économies très dépendantes de la consommation d'énergies fossiles pendant encore des décennies.

Or, la majorité des scénarios montrent que les politiques d'adaptation devront tenir compte d'efforts d'atténuation si elles veulent éviter des situations graves ou extrêmes en 2100 et au-delà. Les tensions qui vont en résulter sont d'ordre géopolitique, car elles dépassent le cadre des États et concernent la communauté planétaire. Un État, a fortiori un ensemble d'États, peuvent-ils durablement soutenir une politique énergétique fondée sur les énergies fossiles, alors que les impacts de la montée de la mer sur leurs propres côtes coûteront plus cher à long terme que la transition énergétique vers une économie décarbonée ? L'enjeu est actuellement tout autant celui d'une démonstration financière et économique des meilleurs choix de scénarios que celui d'une prise de conscience urgente qu'il reste peu de choix et peu de temps pour aller vers des scénarios souhaitables. Il s'agit bien d'une question de géopolitique à l'échelle planétaire.

\section{La géopolitique est très tôt liée à la mer}

\section{\ Sécurité militaire d’abord, commerce et conquête ensuite}

Dès que l'humanité découvre qu'elle peut se déplacer sur l'océan, cet espace apparaît comme une opportunité pour commercer mais aussi pour y déployer des flottes afin de protéger ce commerce, puis étendre son rayonnement par la force. Au v ve siècle avant notre 
ère, Thucydide écrit que la maîtrise de la mer est une composante essentielle de la stratégie militaire. La guerre de Troie commence d'ailleurs par une expédition navale d'envergure. Les stratèges grecs de l'Antiquité savent que « qui tient la mer tient la terre ». La découverte des mines d'argent du Laurion au ve siècle leur permet de financer une flotte capable de faire face à la menace perse en mer Égée (loi navale de Thémistocle). La victoire de Salamine ( -480 av. J.-C.) donne aux Grecs la conservation de la maîtrise de la navigation dans cette mer. La bataille navale d'Actium ( -31 av. J.-C.), qui engage 900 navires, ouvre à Rome la conquête de l'Égypte. De nombreuses batailles navales marquent ainsi des tournants de l'histoire : Lépante, Trafalgar, Tsushima, Midway... L'enjeu naval militaire structure le premier la vision de l'espace maritime sur le long terme car une politique maritime demande, en appui d'une vision sur le long terme, un patient et coûteux travail de mise sur pied, d'entretien et de modernisation d'une flotte, de formation de marins, de plantation de forêts...

Au fil des siècles, les expéditions maritimes relient les côtes, puis les continents. Au début du xve siècle, sept flottes chinoises longent successivement les côtes de l'océan Indien jusqu'à la mer Rouge. Mais ces explorations ne se traduisent pas en l'établissement de comptoirs durables car l'empire du Milieu craint de s'affaiblir en se dilatant. L'avantage géopolitique sur l'Europe est perdu. À la fin du même siècle, le Portugal cherche à contourner le verrou ottoman en Méditerranée, pour rétablir l'accès aux épices des Indes. Il doit donc passer par la mer. Pour s'adapter à la navigation hauturière, la caravelle est mise au point, synthèse des connaissances marines de l'époque. Les expéditions maritimes (Colomb, Vasco de Gama, Magellan...) démontrent alors que presque toutes les terres sont accessibles par la mer. La compétition entre puissances européennes est lancée. Les routes maritimes et des ports d'escale sûrs deviennent des enjeux majeurs pour le commerce et la conquête d'immenses territoires. Exploration et commerce renforcent la justification de l'expansion maritime.

\section{L'Imperium mondial passe par la maîtrise de l'océan}

La mondialisation des échanges commerciaux via la mer, les progrès de la construction navale, et l'avantage stratégique que donne le contrôle des routes maritimes, amènent les grandes puissances à entretenir d'importantes flottes marchandes et militaires. À son apogée, l'Empire britannique prétend "régner sur les vagues », de Londres à Sydney. Les expéditions scientifiques se multiplient au xvIII siècle ; explorateurs et naturalistes font progresser les connaissances, comme Darwin, Humboldt, La Pérouse... non sans arrière-pensées d'influence politique et d'intérêt commercial. L'amiral américain Mahan théorise en 1890 le concept de puissance mondiale et son lien avec la puissance navale. Cette vision de long terme, reprise par le pouvoir exécutif des États-Unis, aboutit à la construction de la plus puissante marine de tous les temps.

Cette logique perdure : la Chine veut devenir aussi une puissance navale majeure, y compris dans le domaine de la recherche marine (Chinese academy of science, 2009). Cette évolution du rapport de l'homme à la mer reste marquée par des enjeux de souveraineté (avec 
plusieurs zones de propriété et de priorité pour les États côtiers), de défense et de sécurité, de supériorité et de diplomatie navale, outil aussi de soft power, de contrôle des détroits, des canaux et des routes maritimes stratégiques, de surveillance des réseaux de câbles sous-marins (où transitent $90 \%$ des communications), de luttes contre les trafics illégaux en mer et de protection des ressources marines (souvent à fins d'exploitation), qu'elles soient vivantes, énergétiques ou minérales. Par ailleurs, le réchauffement des hautes latitudes, et la réduction de la banquise qui en découle, donne à la marine russe une opportunité d'accéder plus facilement à l'Atlantique comme au Pacifique, ce qui rend peu à peu obsolète « la course aux mers chaudes », obsession du pouvoir russe pendant des siècles.

\section{Pertes de territoire et enjeux de souveraineté}

Il faut rappeler que le trait de côte est la limite entre la terre et la mer lors des plus basses marées, appelé aussi le « zéro des cartes marines ». Si la terre s’enfonce (subsidence) ou si le niveau de la mer monte, la ligne de ce zéro de référence se déplace. Une hausse importante de la mer peut donc faire disparaître de vastes territoires si ceux-ci sont plats sur de grandes distances à l'intérieur des terres comme en Floride, en Amazonie, au Bangladesh ou en Thaïlande... En dehors de la haute mer, domaine du droit international régi par de nombreux traités, les mers côtières sont appropriées à partir du trait de côte du continent ou de l'île considéré. Si la hausse de la mer modifie ce trait, les limites des eaux territoriales (12 miles), comme des zones économiques exclusives (ZEE à 200 miles), évolueront, voire disparaîtront si l'île-État est submergée, ce qui est désormais un risque avéré à 2100 pour de petits États insulaires comme les Maldives, les Fidji, les Seychelles... Le droit international n'a pas encore statué sur ce point, car il est empreint de revendications et de conflits potentiels pour des raisons de souveraineté comme d'accès aux ressources. En effet, la « cadastralisation » de plus en plus précise de la mer grâce aux techniques de géolocalisation, permet d'aider à l'arbitrage des enjeux marins côtiers en matière de souveraineté, de droits d'exploitation des ressources, de passage, de statut (aire marine protégée par ex.), etc. Plus gravement, car des vies humaines en dépendent, la disparition d'îles-États pose le problème de leur existence même. En effet, en droit international, « un territoire physique est le titre primordial et absolu de compétence d'un État » (Charte des Nations Unies). Parmi les pays européens, avec des îles et des territoires dans tous les océans, la France dispose d'une capacité unique de présence à l'échelle mondiale. Ainsi, après le passage du cyclone George en 1998 en République dominicaine, la première aide est venue par bateau depuis les Antilles françaises. Or, la montée du niveau de la mer affectera toutes les îles de la Caraïbe, à des degrés divers, notamment la Martinique et la Guadeloupe. Ces îles pourraient devenir des « laboratoires » voire des « modèles » d'adaptation à ce phénomène, couplé à la probable augmentation de la puissance des cyclones dans la région. Avec des compétences administratives, scientifiques, techniques et financières dans tous les domaines impliqués dans cette évolution, la France pourrait contribuer à développer des dispositifs de prévention, d'adaptation et de résilience pour toutes les îles de l'arc caraïbe. 


\section{Vulhérabilité des populations et migrations}

LA SUBMERSION PERMANENTE OU RÉGULIÈRE des zones côtières finit par concerner un nombre croissant de personnes et entraîner des migrations. Dès 1993, la première projection de "réfugiés climatiques » contraints à l'émigration pour cause de submersion et d'insécurité alimentaire donnait 150 millions de personnes entre 1993 et 2050 (Myers, 1993), chiffre réévalué à la hausse (250 millions) en 2018 par le programme des Nations Unies pour l'environnement. Les migrations peuvent conduire à l'exil quand il n'y a plus d'espace disponible ou quand les conditions de vie deviennent insupportables. La majeure partie des pays « à risques » sont situés en Asie et en Afrique, sans compter les îles et archipels de quelques mètres d'altitude, disséminés essentiellement dans l'océan Pacifique et l'océan Indien. Quel statut donner alors aux migrants qui sont obligés de fuir leur pays de manière définitive pour des raisons environnementales ? Quels États accepteront de les recueillir ? Ces migrants iront-ils grossir les populations d'autres zones côtières proches ? L'appellation « migrant climatique » ou « éco-réfugié » a commencé à se répandre et elle est chargée de sens. Mais ce concept, sans support juridique international à ce jour, pose des questions de droit inédites et sans solution simple, même à une échelle de pression minime de migration.

\section{Les impacts sur les politiques économiques et sécuritaires des États}

Au plan économique, une forte hausse du niveau de la mer n'affecterait pas seulement un pays en termes d'investissements à réaliser, mais elle affecterait sa structure même et son avenir. Ainsi, les Pays-Bas commencent à préparer la relocalisation partielle de Rotterdam ( $1^{\text {er }}$ port d'Europe) et à restructurer le tissu urbain et économique dans la plaine centrale, des bouches du Rhin au polder de l'ljsselmeer (exemple de l'habitat flottant à Amsterdam). En effet, dès lors que la submersion marine menace de manière irréversible une région côtière, parfois de manière invisible comme la salinisation des nappes phréatiques, tous les secteurs de l'économie peuvent être affectés : dégradation des infrastructures, des usines et de l'habitat, perte de rendements agricoles, voire abandon de cultures, chute des valeurs patrimoniales et non-assurabilité du bâti, diminution du tourisme, déclin ou perturbation des flux de ressources vivantes suite à des bouleversements d'écosystèmes et les pertes humaines lors de tempêtes côtières. Parfois, la salinisation des sols par l'intrusion marine constitue une opportunité comme dans la transformation de rizières côtières en bassins d'aquaculture d'espèces d'eau saumâtre en Afrique et en Asie. Compte tenu de l'absence de modélisations fiables et précises, les évaluations des coûts économiques d'une hausse de la mer à un horizon donné n'ont qu'une valeur estimative et locale. À l'échelle d'un pays, et de sa place dans une économie mondialisée, la reconfiguration de son trait de côte et l'accroissement des risques côtiers posent des enjeux qui touchent à la politique économique du pays et à ses priorités. Dans ce domaine, l'anticipation vaut mieux que toutes les formes de réaction sous 
réserve que le pays ait une vision claire de long terme en matière de « repli stratégique » et les moyens de le mener à bien

Au plan militaire, l'exemple de l'ouragan Katrina (2005) est révélateur. Ce cyclone de catégorie 5 (1836 morts ; 108 milliards de \$ de dégâts) a déclenché, bien avant le monde politique, la prise de conscience du commandement de l'US Navy que le changement climatique doit être pris au sérieux pour conserver les capacités opérationnelles des bases navales américaines dans le monde. En conséquence, de grands travaux de protection ont été programmés dans plusieurs sites notamment Norfolk (Atlantique Nord), Diego Garcia (océan Indien), Guam (océan Pacifique). Cette évolution est observée dans la plupart des politiques de sécurité maritime des grands États côtiers, surtout dans les régions où le trait de côte sera affecté en profondeur. Par ailleurs, la protection et la surélévation artificielles, et parfois la militarisation, de certaines îles menacées de submersion, afin de conserver la position stratégique et la ZEE associée, est devenue plus fréquente, surtout dans les îles revendiquées par plusieurs pays, comme en mer de Chine (Valantin, 2017).

\section{Les impacts patrimoniaux et culturels}

Le mythe du déluge suivi d'une inondation générale est universel. Les « cités englouties » sont nombreuses, réelles comme Alexandrie ou imaginaires comme Ys. Si certains patrimoines architecturaux côtiers restent hors d'atteinte de la mer comme le Mont-SaintMichel, nombre de villes-musées sont menacées, la plus emblématique en Europe étant Venise. De nombreuses villes côtières à haute valeur patrimoniale sont vulnérables : Abidjan, Alexandrie, Amsterdam, Bangkok, Dacca, Lomé, Miami, Osaka, Shanghaï... De même, des sites remarquables pourraient être submergés notamment dans les grands deltas (Rhône, Pô, Mississipi, Gange, Mékong, Nil, Niger, Amazone...), ainsi que d’innombrables îles basses (Polynésie, Micronésie, Maldives, Frise, Comores, Keys...). Or, il existe des liens forts entre un paysage anthropisé connu et ancien, et l'identité d'un pays (Skyo, 1977). Peut-on imaginer l'Italie sans Venise, les Pays-Bas sans Amsterdam et Rio sans ses plages ? Les coûts, énormes, de protection de ces trésors du patrimoine humain ne pourront être supportés que pour un petit nombre d'entre eux et les arbitrages seront difficiles. À long terme, il est probable que l'on devra le plus souvent laisser faire la nature. On ne fait pas de barrage contre le Pacifique.

\section{Les enjeux de long terme}

En matière de changement climatique et de hausse du niveau de la mer, les conséquences sont aussi d'ordre géopolitique, car tout est lié. Comme ni les mécanismes, ni l'amplitude, ni la vitesse de cette hausse ne sont connus avec précision, c'est le principe de précaution qui devrait prévaloir. Mais le cadre étatique passe encore avant le cadre multilatéral. En conséquence, la réflexion comme l'action restent en deçà de ce qui serait nécessaire de décider et de mettre en œuvre collectivement. Les trois exemples suivants montrent l'intrication des différents phénomènes : 
L'Arctique est riche de ressources, surtout de pétrole et de gaz. Ainsi, en mer de Kara, de nouveaux gisements d'hydrocarbures ont été découverts après ceux de la mer de Barents et de Yamal. L’Arctique recèlerait ainsi des années de consommation mondiale de pétrole et de gaz, dont $70 \%$ en zone russe. Les investissements d'exploitation, considérables, notamment en matière de navires (7 brise-glace dont 3 à propulsion nucléaire) et de réseau ferroviaire, sont soutenus par plusieurs pays d’Asie (Chine, Japon, Corée...). Cet atout énergétique et stratégique justifie la militarisation de l'Arctique russe : flotte, missiles tactiques et balistiques. Ces efforts sont sans équivalent pour les autres pays arctiques, y compris les États-Unis qui n'ont pas de brise-glace nucléaire. Mais l'exploitation du pétrole et du gaz en Arctique aura des impacts croissants et directs sur le changement climatique, la fonte de la calotte polaire et sur la montée du niveau de la mer. Le Conseil de l'Arctique, composé des 8 pays riverains, est actif, mais il n'émet que des recommandations. Il est à craindre que l'accroissement des exportations de l'Asie vers l'Europe et les États-Unis, et l'exploitation accrue des gisements russes conduisent à une fréquence élevée du passage des navires par le passage du Nord-Est toute l'année avec une pollution locale supplémentaire. Quant à l'Antarctique, il ne peut être écarté l'hypothèse de la mise en exploitation de ses ressources fossiles si le protocole de Madrid (1998) était remis en cause comme demandé en 2011 par la Russie.

Sur le plan des ruptures technologiques, on peut imaginer une transition accélérée de l'énergie carbonée vers l'hydrogène produit notamment en mer par hydrolyse en utilisant des énergies marines renouvelables. Mais il est probable que l'inertie des changements de régime énergétique impliquera pendant deux à trois décennies la superposition du charbon, du pétrole, du gaz, du nucléaire et des énergies renouvelables. Cela ne sera pas sans effet sur les fonctions écosystémiques de l'océan comme l'absorption de chaleur, qui est responsable d'environ un tiers de la hausse du niveau de la mer et celle de « puits de carbone » de la biomasse marine (déplacement du carbone de l'atmosphère vers le fond de l'océan ou la chaîne alimentaire jusqu'à l'homme).

Liées au changement climatique, les modifications des systèmes physiques de circulation des masses océaniques pourraient avoir des effets de ralentissement ou d'accélération de courants, notamment dans l'océan Pacifique, avec des conséquences parfois catastrophiques en termes de cyclones et de précipitations. Ces phénomènes se traduisent sur les côtes impactées en submersions et inondations.

La dimension géopolitique du choix des priorités de recherche apparaît ici clairement. La multiplicité des interactions mises en mouvement par la hausse du niveau de la mer justifie de développer des recherches interdisciplinaires en associant tous les acteurs. L'analyse prospective pourrait alors être prolongée par un diagnostic précis des besoins en recherche dans tous les domaines, y compris leurs interfaces, puis par une planification des thèmes de recherche à renforcer. Ces recherches faciliteraient la sélection des moyens à mettre en œuvre pour anticiper, éviter, réduire et compenser les effets des submersions locales ou généralisées, tout en limitant les inégalités sociales face aux risques. Il faudra aussi se préparer à gérer inévitablement des situations de crise. Cette approche 
en recherche ne se conçoit que dans le cadre de la coopération internationale, le seul pertinent compte tenu de l'échelle des enjeux. De fait, les tensions croissantes sur l'occupation des zones vulnérables se traduiront, tôt ou tard, par des pressions de migration transfrontalières, y compris au sein de l'Europe.

\section{Enseignements à l'échelle de la France comme du monde}

En France, IL EXISTE de nombreux plans et comités à toute échelle, de la commune à l'État, pour anticiper et traiter les risques de submersion/inondation. Mais si les scénarios démontrent l'utilité des « replis stratégiques » ou même de "restructuration territoriale » limitée dans l'espace, il est difficile de les mettre en œuvre au plan politique, technique et financier. Les raisons sont connues : incertitudes des projections, manque de représentation concrète des risques, conservatisme des habitants, prudence des élus, dilution des responsabilités, complexité des structures administratives et des réglementations, attentisme des assureurs... Il est probable que seules les catastrophes comme celle de la tempête Xynthia en France (2010), et leurs coûts croissants associés, pourraient faire bouger les choses. Mais avant qu'elles se produisent, comment quantifier les risques ? Sans schéma de financement acceptable par les différentes parties prenantes comment financer et organiser le déplacement de dizaines de maisons, d'entreprises agricoles, voire de villages entiers ? Les meilleurs rapports d'analyse et de diagnostic, comme Acclimaterra en région Nouvelle-Aquitaine (Le Treut et al., 2018), ne peuvent qu'éclairer les acteurs sur les décisions à prendre.

L'égocentrisme et la vision restreinte au court terme s'expriment à l'échelle des États, malgré nombre de structures, comme des réseaux de mesure et des observatoires internationaux, des modèles standardisés de simulation du climat, des recherches en coopération à toute échelle. Les capacités des États à mobiliser des budgets, souvent considérables compte tenu de l'ampleur des travaux à mener, sont très diverses. Avec un produit intérieur brut 11 fois supérieur à celui du Bangladesh (161 millions d'habitants) et pour un linéaire côtier bien moins vulnérable, la Californie (39 millions d'habitants) dispose d'une puissance financière très supérieure pour traiter cette menace. Mais si la Realpolitik prévaut actuellement dans les choix des États, l'amélioration des outils de modélisation et de simulation des situations à venir devrait permettre de mieux chiffrer les paramètres clefs de la hausse de la mer et de ses conséquences. Cela contribuerait à sélectionner les meilleures stratégies en termes de coûts-bénéfices comme de risques. Cette approche faciliterait l'objectivation des conséquences de choix de gestion de l'eau sur un bassin versant traversant plusieurs frontières. L'ampleur des conséquences multiples de la hausse du niveau de la mer explique pourquoi la dimension géopolitique apparaît fréquemment dans l'évaluation des risques liée à ce phénomène. II est logique de penser que cette dimension va prendre de plus en plus d'importance en raison même des enjeux croissants que révèle cette hausse. 


\section{Conclusion}

"Prenons le changement par la main avant qu'il ne nous prenne par la gorge.» (W. Churchill)

EN RAISON MÊME DE LA COMPLEXITÉ des enjeux, de la variété des échelles géographiques et de la multiplicité des conséquences de la hausse du niveau de la mer à l'échelle mondiale, la prospective a été mobilisée pour apporter une contribution à la nécessaire réflexion stratégique sur les choix à faire pour s’y préparer. Les focus régionaux sur trois régions très différentes ont servi à montrer à la fois la diversité des contextes et la nécessité commune d'anticiper les situations probables à venir sur les littoraux afin de maîtriser au mieux les coûts comme les crises. Les scénarios construits au terme d'une analyse croisée de 23 variables motrices conduisent à des conclusions claires et globalement préoccupantes. En conclusion, il est utile de souligner leurs enseignements et d'en tirer des recommandations pour l'action.

\section{Des tendances lourdes et des scénarios inquiétants}

IL CONVIENT D'ABORD DE RAPPELER que l'humanité, qui gagne un million d'habitants tous les cinq jours et entretient sa croissance économique à partir d'énergies à $80 \%$ d'origine fossile, perturbe de plus en plus gravement le « système Terre » dans de nombreux domaines : le réchauffement climatique, la qualité de l'air et des eaux, la disponibilité en terres arables, la réduction du couvert forestier, la perte de biodiversité dans tous les écosystèmes, l'acidification de l'océan... On a pu ainsi proposer de qualifier d' "Anthropocène », à la suite de Paul Crutzen, prix Nobel de chimie, l'ère dans laquelle nous vivons depuis la révolution industrielle du xvIII ${ }^{\mathrm{e}}$ siècle.

L'océan et les littoraux sont emblématiques de cette évolution inquiétante dont les symptômes de dégradation sont multiples et se renforcent parfois l'un l'autre, notamment en matière de ressources vivantes.

Considérer ces espaces comme « la dernière frontière sauvage de la planète » susceptible d'une approche raisonnable prenant en compte le long terme est parfois présenté comme l'idéal d'un projet collectif pour l'humanité, une sorte d' « utopie salvatrice » (Habermas, 2011). En fait, la majorité des travaux scientifiques, via de nombreuses structures officielles (GIEC, IPBES, UICN...), comme les enquêtes de multiples autres sources (Cury et Miserey, 2008 ; Urbina, 2019), convergent plutôt vers le diagnostic d'une dystopie de l'océan mondial, espace d'un « mal commun » plutôt qu'un « bien commun ». Il suffit de citer quelques chiffres pour le vérifier : plus de $20 \%$ de pêche illégale, 70 \% de la flotte 
de commerce mondiale sous pavillon dit « de complaisance », persistance de formes d'esclavage de marins dans de nombreux pays, et environ 160 milliards de dollars de chiffre d'affaires issus d'exploitations et de trafics illégaux dans le seul domaine maritime. Cette situation concerne aussi les littoraux sous de nombreuses formes : destruction de mangroves, pêche à l'explosif dans les zones coralliennes, pollutions massives à la côte...

La montée du niveau de la mer est un phénomène connu et mesuré depuis des décennies. Il est aujourd'hui inéluctable et en accélération. Il exacerbe les risques de mauvaise gestion des espaces comme des ressources. Ses causes ne sont pas actuellement maîtrisées, bien au contraire. Cette étude prospective a exploré une large gamme de scénarios d'évolution possibles en fonction de deux critères majeurs : les choix de gouvernance sur les efforts d'adaptation du littoral et ceux concernant l'atténuation du changement climatique. On observe que sur huit scénarios, cinq mènent à des situations qualifiées d' " extrême » ou de " grave », même en tenant compte de changement profond de stratégie vers 2050-2060. Par ailleurs, la situation tendancielle actuelle (de type « Fragmentation persistante » des politiques des États) mènerait à une situation « extrême » si elle devait perdurer encore quelques décennies.

Les situations plus acceptables en 2100 (états physiques de type «modéré » et « sérieux »), issues de trajectoires plutôt stables, sont l'aboutissement de conjectures assez peu probables car elles combinent des politiques vertueuses, fermes et continues à l'échelle mondiale. Comme ces conjectures sont des ruptures fortes par rapport aux tendances lourdes actuelles, les probabilités d'occurrence de ces deux scénarios devraient être logiquement considérées comme faibles.

Il en résulte qu'il est réaliste d'envisager comme une hypothèse plausible une montée du niveau de la mer de l'ordre de 1 à 2 mètres d'ici 2100, avec la persistance d'une dynamique d'accélération. Cela aurait des conséquences considérables sur la majeure partie des côtes basses habitées, où se concentrent précisément de nombreux secteurs de l'activité humaine. Seraient concernés des domaines vitaux comme les grandes métropoles, la sécurité des personnes vivant le long des côtes, la stabilité des constructions de tout type, la production agricole, l'accès à l'eau douce, mais aussi le profil même des fleuves. De plus, les projections de hausse doivent également intégrer un accroissement de la puissance des événements météorologiques extrêmes sur le littoral, notamment en zone tropicale. Au fil des rapports des collectifs internationaux d'experts, les connaissances scientifiques confirment que le plus probable est que la situation devrait continuer d'empirer au-delà de 2100 et pendant plusieurs siècles.

\section{Des marges de manœuvre}

LA RÉACTIVITÉ DES DÉCIDEURS (responsables politiques, entreprises, consommateurs...) apparaît comme un facteur essentiel de préservation des situations à des états « modéré » et " sérieux » en 2100. Si l'on vise au terme du siècle l'un de ces deux états et que l'on 
considère que le monde se situe actuellement sur une trajectoire de fragmentation, le changement de politique doit être rapide, radical et généralisé avant 2040 pour trois scénarios sur huit (Maîtrise climatique, Sobriété et anticipation, Villes résilientes). Pour les cinq autres trajectoires, le changement sera trop tardif pour éviter des situations de type « grave » ou « extrême ».

Les scénarios mettent ainsi en scène des tensions exacerbées entre les échelles d'action mondiales et littorales. L'atténuation du changement climatique reste liée à une hypothétique gouvernance mondiale, tandis que les dynamiques d'adaptation littorale dépendent surtout d'autorités nationales, régionales ou urbaines. Or les acteurs des zones littorales n’ont qu'une faible capacité à agir sur la mise en œuvre à l'échelle mondiale de mécanismes d'atténuation, dont l'absence ou la présence déterminera pourtant l'ampleur de l'élévation du niveau de la mer à laquelle ils devront faire face à l'avenir.

Par ailleurs, cette crise climatique globale de l'Anthropocène est autant un risque de déstabilisation des écosystèmes et de leurs fonctions associées que des activités humaines (Bourg et al., 2013). Aussi, l'inventaire des scénarios d'évolution possible des socio-écosystèmes marins compte moins que la prise de conscience du rôle essentiel de l'océan dans l'avenir du climat planétaire. Il s'agit aussi d'une course contre la montre avec des risques de seuils irréversibles identifiés comme la fonte des glaces aux pôles et toutes les conséquences qu'elle pourrait avoir sur la physique des océans, la biodiversité, ou les pertes de fonction de certains écosystèmes. Un des rôles de la connaissance scientifique est d'éclairer continûment les systèmes de gouvernance sur les conséquences de leurs choix en matière de réduction des énergies fossiles, de politique industrielle, de développement de certains modes de transport plutôt que d'autres, de modèles économiques dans l'agriculture, l’industrie et l'énergie (Euzen et al., 2019).

Le dernier rapport de l'OCDE (2019) sur les risques liés à la submersion marine montre que, si la hausse du niveau de la mer reste de l'ordre du mètre, les conséquences en termes économiques pourraient être à peu près gérées à l'échelle de la majorité des pays concernés. En revanche, dans le cas de scénarios plus graves (1,3 à $2 \mathrm{~m}$ ), la réponse pertinente devrait changer d'échelle notamment en matière d'anticipation des phénomènes, d'adaptation des infrastructures et des villes côtières et d'organisation de la transition de l'économie côtière.

\section{S’adapter, action nécessaire mais pas suffisante}

LES SCÉNARIOS ÉLABORÉS DANS CETTE ÉTUDE montrent qu'un changement de cadre conceptuel est nécessaire dans les stratégies d'adaptation, du fait de l'irréversibilité des changements subis mais aussi de l'absence de solutions technologiques disponibles pour y faire face durablement dans une situation d'emballement du changement climatique (Magnan et Duvat, 2015). 
Les mesures d'adaptation au risque d'élévation marine, telles que la construction de digues, le rechargement du cordon littoral, le renforcement des écosystèmes littoraux et côtiers ou le repli stratégique sont à concevoir en fonction des spécificités des situations littorales (morphologie côtière, population, urbanisation, infrastructures, écosystèmes) et des cultures locales du risque. Dans tous les cas, agir conjointement en limitant le changement climatique et en anticipant la hausse du niveau marin permet de réduire les coûts de l'adaptation littorale et de limiter l'ampleur et la fréquence des crises. De plus, étant donné que le niveau d'élévation des mers auquel il convient de s'adapter s'avère très incertain après 2050, la réversibilité des mesures d'adaptation est à privilégier afin d'écarter les risques de mal-adaptation des zones littorales. Il s'agit notamment de développer des solutions basées sur la nature et de s'appuyer sur des démarches participatives incluant les acteurs locaux (Stammer et al., 2019).

Mais les scénarios introduisent également un changement de paradigme dans la gestion du risque. Auparavant, il s'agissait de vivre dans un « monde incertain », en tenant compte des incertitudes et en adaptant les stratégies d'action pour essayer de maîtriser les situations. Dans le monde à venir, il pourrait s'agir pour certaines régions du monde de vivre dans un état de crise quasi permanente, de vivre avec des catastrophes récurrentes, en tentant de faire face à leurs conséquences et d'en réduire les impacts. Pour d'autres régions du monde, la prise en compte de plus en plus précoce des catastrophes potentielles liées à la hausse du niveau de la mer et des mesures à adopter en conséquence pourraient permettre de réduire les coûts humains, économiques et politiques. Le parallèle avec les cyclones tropicaux est éclairant : alors que ces phénomènes ont été longtemps considérés comme des calamités meurtrières inévitables avec lesquelles il fallait vivre, de grands progrès ont été faits dans les dernières décennies pour connaître leurs conditions de déclenchement, leurs paramètres à tout moment et leur trajectoire. Ces connaissances, traduites ensuite en termes de mesures de protection civile et de procédures d'information du public ont permis d'en réduire considérablement les impacts.

De manière concrète, actuellement, tout responsable d'aménagement sur un littoral vulnérable peut préparer ses choix en s'appuyant sur trois dimensions. Il doit d'abord prendre en compte le temps long, c'est-à-dire l'échelle de plusieurs décennies car anticiper coûte toujours moins cher. Des outils de modélisation de plus en plus précis permettent aujourd'hui de projeter diverses situations à différents horizons temporels. Ensuite, il est indispensable de mener un calcul coûts-bénéfices sur les options d'aménagement en intégrant les services rendus par la nature. Enfin, il doit tenir compte des emboîtements des échelles d'aménagement, car une commune littorale s'intègre dans plusieurs systèmes territoriaux, de la communauté de communes jusqu'au bassin versant. Cette intégration vaut aussi pour le financement des aménagements et notamment celui de la restructuration du patrimoine sous toutes ses formes : sols agricoles, infrastructures collectives, activités industrielles, bâti ancien et récent. Un repli stratégique anticipé, donc mieux ordonné, coûte toujours moins cher qu'un abandon contraint dans l'urgence. La clef de répartition des financements des relocalisations, le rôle des 
services assurantiels et la coordination des décisions doivent être discutées avec tous les acteurs et suffisamment tôt pour préparer la phase de transition en minimisant les risques de crise et de catastrophe (CCR, 2018).

Toutes ces dynamiques d'adaptation n'ont de sens sur le long terme que si elles sont couplées à des politiques ambitieuses d'atténuation du changement climatique (OECD, 2019). Ces politiques devront être mises en œuvre tôt ou tard, comme le montrent clairement les scénarios de cette étude.

\section{Des raisons d'espérer}

Parce que le Changement Climatique est global, irréversible, plus rapide que les temporalités politiques, sa maîtrise exige un « nouveau contrat social » à l'échelle mondiale. Les enjeux de gouvernance climatique sont autant de défis pour le monde politique, celui de l'économie et les sociétés. Et il y a urgence, comme le rappelle une note de la Banque centrale européenne, incluant la submersion marine dans les grands risques systémiques affectant l'Europe dans les trois décennies à venir et justifiant de tester la résistance de l'économie au changement climatique sur 30 ans (BCE, 2021).

Ces perspectives, plutôt sombres, justifient d'accroître les efforts en matière de recherches interdisciplinaires en y associant tous les acteurs. Il s'agit de comprendre, mesurer et projeter le phénomène, évaluer ses impacts, engager les transitions littorales et urbaines, préciser et mettre en œuvre les moyens de réduire ou de compenser les effets des submersions, limiter les inégalités face aux risques et se préparer enfin à gérer les situations de crise. Le caractère d'urgence et d'universalité de ces enjeux n'est plus à démontrer. De fait, un tel bouleversement n'a pas d'équivalent dans l'histoire du monde à cette échelle de temps (Cassier et al., 2017).

Mais les scénarios du pire ne sont pas certains. Il faut rappeler comme un leitmotiv qu'une atténuation précoce et massive du changement climatique, qui suppose une action rapide concertée à l'échelle mondiale et poursuivie durant des décennies, sinon des siècles, sans retour en arrière, limiterait l'élévation du niveau de la mer et, par conséquent, les efforts d'adaptation littorale nécessaires. Le coût global de l'inaction devrait peser aussi dans les décisions. La meilleure solution reste celle de l'apprentissage d'un nouveau modèle de coopération internationale, commençant par un ambitieux dispositif de sensibilisation, d'éducation et de compréhension collective de ces enjeux environnementaux. Si l'on considère cette voie, certains signes sont encourageants, comme l'accord de Paris de 2015 sur le climat (COP 21), ou les pressions croissantes pour la généralisation de la mesure systématique de l'empreinte de l'activité de toute entreprise sur l'environnement et de tout produit, en termes de bilan carbone et de recyclabilité. Cette vision volontariste exige l'implication soutenue de tous les acteurs de la société, et d'une majorité de pays, et pas seulement l'affichage d'ambitieux objectifs de développement durable dont la mise en œuvre réelle est sans cesse reportée. 
La hausse du niveau de la mer constitue un indicateur très synthétique de multiples phénomènes à l'œuvre. Elle affecte une fraction grandissante de l'humanité, sur des territoires paradoxalement de plus en plus convoités, mais aussi de plus en plus vulnérables, en raison de l'accélération du phénomène (Geisler and Currens, 2017). Ce paradoxe résume bien la contradiction du modèle général de la croissance, à l'évidence non durable. Et aujourd'hui, aucune technologie ne pourrait enrayer rapidement un phénomène d'une telle ampleur et d'une aussi grande inertie.

Ainsi, les risques de submersion pourraient contribuer à la nécessaire prise de conscience qu'il est temps d'agir collectivement sur l'ensemble des causes. Cette prise de conscience n'a de sens et d'efficacité que si elle s'étend à l'échelle mondiale, du citoyen aux organisations internationales, en mettant en mouvement toute la sphère économique (Afriat et Theys, 2018). Cependant, il faut se garder d'idéaliser « la communauté internationale ». L'action des institutions internationales n'est que la résultante de la stratégie des États, et notamment des plus puissants. Force est de constater que, depuis 20 ans, tous les États ne sont pas disposés, à transformer leurs secteurs d'activité les plus émetteurs et leurs consommations de ressources pour réduire rapidement les émissions de gaz à effet de serre. En vue de résoudre cette impasse, il s'agit de reconnecter deux territoires : le territoire où nous vivons et le territoire dont nous vivons, soit le territoire juridique et politique de l'État-nation et le territoire économique et écologique de l'espace requis pour mobiliser les biens que l'on consomme (Charbonnier, 2020). Les sociétés civiles, les communautés locales, les collectivités territoriales ont, aux côtés de l'État, un rôle central dans cette évolution qui concerne à la fois l'exploration des problèmes qui les affectent, et la recherche et la mise œuvre de solutions adaptées à ces problèmes. Car comme le rappelle Bruno Latour (2020) en reprenant les conceptions de John Dewey, «l'État n'a pas d'autre accès à la connaissance du bien commun que les canaux offerts par l'exploration de la société civile ».

Il revient donc à ces collectifs à diverses échelles la responsabilité de transformer cette menace en opportunité de solidarité mondiale et de coresponsabilité intergénérationnelle. Ce serait la reconnaissance du fait que la mer est un bien commun vital de la planète et de l'Humanité. 


\section{Remerciements}

Les auteurs tiennent à remercier les trois experts qui ont contribué, chacun dans leur domaine, à la relecture critique de ce livre, François-Xavier Deniau, Paul de Puybusque et Christophe Robinne, ainsi que Robert Slomp pour la partie concernant les Pays-Bas, Nicolas Rocle et Hervé Le Treut pour la partie Nouvelle-Aquitaine, Christian Valentin et Sylvain Ouillon pour la partie Vietnam. 


\section{Annexe : \\ Méthodologie}

Le large éventail des vitesses de montée du niveau de la mer et la variété des conséquences et des réactions possibles justifient le recours à la méthode des scénarios basée sur l'analyse morphologique. Cette méthode classique et éprouvée en matière de prospective est particulièrement bien adaptée à la complexité de la question posée et les fortes incertitudes qui l'accompagnent

Elle implique un travail en ateliers prospectifs à dire d'experts afin de bien circonscrire le problème posé et le système étudié, une analyse structurelle afin d'en déterminer les variables clefs, une analyse morphologique afin de balayer le champ des possibles et construire des scénarios pertinents, cohérents et vraisemblables.

Nous nous sommes intéressés ici aux conséquences et anticipations possibles du phénomène de montée du niveau de la mer à l'échelle mondiale, puisqu'il s'agit d'un phénomène global et d'ici 2100 afin de prendre en compte l'inertie du phénomène, mais aussi l'éventualité de son emballement sur cette période (par exemple, dans l'hypothèse d'une fonte totale de la calotte glaciaire).

Les personnes impliquées ainsi que les étapes de cette méthodologie sont décrites ci-après.

\section{Équipe projet et organisation des travaux}

Afin de mener cette étude, trois entités aux rôles complémentaires n'ont cessé d'interagir. Leur composition est détaillée ci-dessous.

\section{IÉquipe d'animation :}

- Nicolas de Menthière (INRAE), Olivier Mora (INRAE) et Denis Lacroix (Ifremer), co-animateurs du Groupe Transversal (GT) Prospective d'AllEnvi ;

- Christophe Didier (INERIS) et Murielle Lafaye (Cnes), membres du Groupe Transversal (GT) Prospective d'AllEnvi ;

- Audrey Béthinger (INRAE), cheffe de projet.

\section{IÉquipe Information Scientifique et Technique :}

- Marie-Hélène Pépin (Météo France), Morgane Le Gall (Ifremer) et Pascale Hénaut (Irstea). 


\section{Groupe d'experts :}

- Bernardot Marc (Université du Havre) ;

- Bertin Xavier (CNRS/GT Mer AllEnvi) ;

- Carnus François (BRLIngénierie) ;

- Cattaneo Antonio (Ifremer/GT Risques AllEnvi);

- Devès Maud (AFPCN/IPGP) ;

- Dörfliger Nathalie (BRGM) ;

- Durand Gaël (CNRS) ;

- Duval Anne-Marie (Cerema) ;

- Guedon Sylvine (IFSTTAR) ;

- Hérivaux Cécile (BRGM) ;

- Le Cozannet Gonéri (BRGM) ;

- Lobry Jérémy (IRSTEA) ;

- Meerpoel Matthieu (ICL) ;

- Meyssignac Benoit (LEGOS) ;

- Petitet Sylvain (Cerema) ;

- Rocle Nicolas (INRAE) ;

- de Rouw Anneke (IRD) ;

- Salas y Melia David (Météo France) ;

- Vagner Marie (CNRS) ;

- Valentin Christian (IRD/GT Sols AllEnvi).

Les travaux ont été menés sur neuf ateliers d'une journée entre juin 2017 et fin novembre 2018, selon le programme suivant :

Atelier 1 : identification des conséquences potentielles de la hausse du niveau de la mer ;

Atelier 2 : identification des variables influentes ;

Atelier 3 : hypothèses d'évolution des variables à $2100(1 / 2)$;

Atelier 4 : hypothèses d'évolution des variables à $2100(2 / 2)$;

Atelier 5 : scénarios d'évolution à 2100 (1/3) ;

Atelier 6 : scénarios (2/3);

Atelier 7 : scénarios (3/3);

Atelier 8 : focus territoriaux ;

Atelier 9 : enjeux pour la recherche.

La plupart des ateliers ont été introduits par des exposés spécialisés permettant d'apporter à l'ensemble du groupe d'experts un éclairage sur une thématique particulière et utile à l'étude. Quelques auditions complémentaires ont été menées par l'équipe d'animation en dehors des ateliers. 


\section{Liste des intervenants en atelier :}

- Anny Cazenave (Cnes) sur le niveau de la mer : du global au local, observations et causes;

- Gaël Durand (CNRS, Univ. Grenoble-Alpes) sur la contribution de la cryosphère à la hausse de la mer ;

- David Salas y Mélia (Météo France) sur les projections de la hausse du niveau de la mer;

- Claire Jolly (OCDE, Unité « Politique de l'innovation pour l'espace et les océans ») sur la montée des eaux : quelles conséquences socio-économiques?

- Jacques Rougerie (Institut de France) sur la montée des eaux : quelles conséquences urbaines et en aménagement côtier?

- Alexandre Magnan (IDDRI) sur le passage des trajectoires de vulnérabilité aux trajectoires d'adaptation ;

- Manh Largemain et Jérôme Chardon (ministère des Armées) sur les grands enjeux et les conséquences « défense » de la hausse du niveau de la mer ;

- François Gemenne (Univ. de Liège et Université de Versailles Saint-Quentin-en-Yvelines) sur la hausse du niveau des mers et les migrations des populations ;

- Robert J. Nicholls (Univ. de Southampton) sur l'aménagement des deltas face au changement climatique.

\section{Liste des intervenants en audition :}

- Jean-Louis Oliver (Académie des Sciences d'outre-mer et Académie de l'eau) ;

- Stéphane Penet (Fédération française de l'Assurance);

- Jean-Paul Billaud (CNRS, Université Paris-Nanterre, Laboratoire des dynamiques sociales et recomposition des espaces LADYSS) ;

- Jean-Louis Valantin (Consultant en géopolitique, auteur de Géopolitique d'une planète déréglée, Le Seuil, 2017).

\section{Les étapes de la construction du système}

La construction du système prospectif: il s'agit d'identifier les variables les plus influentes sur l'évolution de la question posée et de les regrouper en grandes composantes. Lors du premier atelier, une séance de "brainstorming " a mis en évidence le nombre et la diversité des conséquences de la hausse du niveau de la mer. L'équipe d'animation a proposé, sur cette base, un regroupement de ces éléments en composantes. Cette proposition a été mise en débat lors de l'atelier 2, au cours duquel différentes variables ont été identifiées pour chacune des composantes.

L'analyse rétrospective de l'évolution récente de chacune des variables et composantes retenues en s'appuyant sur la bibliographie : elle permet de mettre en avant les tendances marquantes des dernières décennies, ainsi que les points de rupture possibles et les signaux faibles d'évolutions qui pourraient avoir lieu dans le futur. 
La formulation d'hypothèses contrastées d'évolution à 2100 pour chacune des variables retenues : toutes les variables des six composantes ont été traitées successivement lors de deux ateliers. Une synthèse de l'analyse rétrospective des variables de chacune des composantes a permis au groupe d'experts de formuler des hypothèses retravaillées et synthétisées ensuite par l'équipe d'animation.

La construction du tableau morphologique : il s'agit de la matrice regroupant l'ensemble des hypothèses pour chacune des variables, elles-mêmes regroupées en composantes. Cette matrice constitue l'outil de travail permettant l'émergence des scénarios.

La construction des scénarios : en sélectionnant dans le tableau morphologique une hypothèse pour chacune des variables et en les associant afin de construire des récits du futur cohérents, plausibles et contrastés.

Les trois focus territoriaux ont été traités chacun par un binôme. L'analyse a été faite sur une base essentiellement bibliographique pour le Vietnam, à partir de la bibliographie et d'une expertise locale pour la Nouvelle-Aquitaine et les Pays-Bas, complétée par une visite collective de deux jours à sur le terrain pour les Pays-Bas.

\section{Structuration des visions d'avenir et schéma de positionnement des scénarios sur un plan}

LE NOMBRE DE SCÉNARIOS FINALEMENT RETENUS (8) a conduit le groupe projet à proposer une structuration des visions du futur dont ils étaient porteurs en les regroupant en familles. En outre, pour faciliter leur présentation, un schéma de positionnement de l'ensemble des scénarios sur un plan selon deux axes a été recherché. Plusieurs jeux d'axes et modes de représentation ont été proposés et discutés avant d'aboutir à celui finalement retenu pour une présentation synthétique de l'ensemble des scénarios faisant apparaître, pour chaque scénario, à la fois la trajectoire suivie et ses inflexions éventuelles et le contexte physique d'élévation des mers auquel il conduit (cf. chapitre I).

\section{Les composantes du système}

Chaque composante du système se définit par un ensemble de variables. La liste ci-dessous présente le découpage en composantes (en gras) et variables de l'ensemble du système.

\section{Population}

P1 - Part de la population exposée

P2 - Migrations internes et internationales

$P_{3}$ - Degré de vulnérabilité sanitaire des populations 


\section{Urbanisme et infrastructures}

U1 - Dynamiques urbaines

U2 - Niveau de résilience des infrastructures

U3 - Adaptations des zones littorales exposées

\section{Environnement et ressources naturelles}

EN1 - État de la ressource en eau douce (quantité et qualité)

EN2 - État des sols (salinisation, érosion...)

EN3 - Dynamiques des écosystèmes littoraux et côtiers (habitats, biodiversité)

EN4 - Modification du trait de côte

\section{Agriculture et alimentation}

A1 - Disponibilité en terres agricoles

A2 - Systèmes de production agricole

A3 - Poids de l'aquaculture et de la pêche dans l'alimentation

A4 - Sécurité alimentaire (disponibilité, accès, utilisation, stabilité)

\section{IÉconomie littorale}

EC1 - Économie littorale (poids, capacité de transformation, degré de dépendance aux écosystèmes de l'économie)

EC2 - Solidarités et mutualisation (pour adaptation et gestion des crises)

\section{Gouvernance littorale}

G1 - Prise de conscience des risques littoraux (gouvernants et société)

G2 - Réactivité et degré d'engagement (proactivité, acceptabilité, éducation)

G3 - Niveau de coordination et de mutualisation (échelles locale et globale)

\section{Contexte global}

$\mathrm{C}_{1}$ - Croissance économique mondiale

C2 - Mix énergétique (dépendance aux énergies fossiles et au commerce international)

C3 - Prise de conscience de l'enjeu climatique à l'échelle globale (gouvernants et société)

G4 - Gouvernance géopolitique globale 


\section{Le tableau morphologique}

Le TABLEAU MORPhologique reprend le découpage du système prospectif en un système littoral et un contexte global décrit dans le chapitre précédent, auquel s'ajoute un contexte physique d'élévation du niveau de la mer (cf. chapitre I).

Chaque ligne correspond à une variable d'une composante et décrit l'ensemble des hypothèses d'évolution à l'horizon 2100 construites par le groupe d'experts.

Le tableau morphologique a servi à construire les huit scénarios décrits au chapitre I. Chaque scénario se définit par une combinaison d'hypothèses d'évolution des variables, cette combinaison décrivant un état du système en 2100. Pour cela, on sélectionne pour chaque variable une hypothèse d'évolution, puis l'on recherche, pour toutes les autres variables, des hypothèses cohérentes entre elles, en privilégiant le sens général de la combinaison et sa plausibilité.

\section{Construction des scénarios}

Deux méthodes de construction des scénarios ont été menées en parallèle par deux sous-groupes du groupe d'experts.

Dans la première méthode, le choix préalable d'un des quatre contextes physiques d'élévation du niveau de la mer en 2100 (cf. chapitre I) constitue l'élément initiateur et structurant d'un scénario. On cherche alors à lui associer des hypothèses cohérentes pour chaque variable de chaque composante. L'ensemble de ces hypothèses sélectionnées construisent un scénario cohérent avec le contexte physique initialement choisi. Ce travail est répété pour chaque contexte. Plusieurs scénarios peuvent être construits pour un même contexte.

Dans la seconde méthode, le choix d'une hypothèse importante pour une variable motrice majeure du tableau morphologique, et ce quelle que soit sa place dans ce tableau, constitue l'élément structurant et la base du récit. On s'attache alors à rechercher dans le tableau des hypothèses cohérentes avec cette hypothèse initiale. On construit ainsi un scénario du système « littoral » cohérent et plausible. Puis on détermine, à la lumière du récit construit, lequel des quatre contextes physiques correspond le mieux au scénario ainsi bâti.

Les scénarios produits par chacun des deux sous-groupes ont été présentés et discutés au sein du groupe d'experts au complet. Parmi la quinzaine de scénarios initialement produits, les scénarios proches ont été fusionnés afin d'aboutir à un ensemble de scénarios qui, sans être exhaustif, couvre bien le champ des futurs possibles. 


\section{Glossaire}

APEC (Asia-Pacific Economic Cooperation ou Forum de la coopération pour l'Asie-Pacifique) : organisation intergouvernementale qui vise à faciliter la croissance économique, la coopération les échanges et les investissements dans cette région.

ASEAN (Association of South-East Asia Nations) : Association des nations de l'Asie du Sud-Est. Organisation politique, économique et culturelle qui regroupe 10 pays d'Asie du Sud-Est.

Dystopie : récit de fiction, qui décrit un monde et une société imaginaires dans lequel il n'est pas possible d'être heureux et dont l'avenir est sombre ; son contraire est l'utopie. Étiage : débit minimal d'un cours d'eau (calculé en général sur une moyenne de 20 ans). GES (Gaz à Effet de Serre) : ensemble des gaz de la haute atmosphère qui retiennent la plus grande partie du rayonnement infrarouge émis par la terre. On ramène leur effet en équivalent de gaz carbonique car c'est celui qui est le plus important en quantité ; l'unité de mesure est la gigatonne (milliard de tonnes ou Gt). Elle permet d'évaluer le stock déjà présent (environ $2000 \mathrm{Gt}$ ) et les flux émis chaque année principalement par les activités humaines (environ $35 \mathrm{Gt}$ ).

IPBES (Intergovernemental science policy Platform on Biodiversity and Ecosystem Services) : Plateforme intergouvernementale scientifique et politique sur la biodiversité et les services écosystémiques.

IPCC (International Panel on Climate Change) : Groupe international pour l'étude du climat (GIEC).

NOAA (National Oceanic and Atmospheric Agency) : Agence des États-Unis d'Amérique pour la recherche sur l'océan et l'atmosphère.

RCP (Representative Concentration Pathway) : scénario de bilan thermique terrestre jusqu'en 2300, lié à différentes concentrations de gaz à effet de serre (en équivalent $\mathrm{CO}_{2}$ ) ; le GIEC en distingue quatre $: 2.6 ; 4.5 ; 6.0 ; 8.5$ depuis son $5^{\text {e }}$ rapport (2014). Ces valeurs sont données en énergie par mètre carré $\left(\mathrm{W} / \mathrm{m}^{2}\right)$. Elles mesurent le surplus d'énergie ("forçage ») apporté sur terre depuis la période préindustrielle.

Subsidence : phénomène d'abaissement à grande échelle du sol dû, le plus souvent, à des déficits d'apport de matière (eau, sédiments...) et parfois combinés à des extractions massives de matière (pétrole, gaz, eau, granulats).

UICN (Union internationale pour la conservation de la nature) : organisation internationale accueillant des États comme des organisations non gouvernementales et dédiée à la protection de l'environnement. Elle gère notamment la liste rouge des espèces à protéger. WUP (World Urbanization Prospects) : Groupe d'études des Nations Unies sur la population mondiale. 


\section{Bibliographie}

Afriat C., J. Theys (coord.), 2018. La grande transition de l'humanité. FYP Éditions. 267 p.

Aronson J., Milton S. J., Blignault J. (eds), 2007. Restoring natural capital: science business and practice. Island Press. Washington DC. USA.

Azevedo de Almeida B., Mostafavi A., 2016. Resilience of Infrastructure Systems to Sea-Level Rise in Coastal Areas : Impacts, Adaptation Measures, and Implementation Challenges. Sustainability, 8 (11), 1115.

Bamber J. L., Oppenheimer M., Kopp R. E., Aspinall W. P., Cooke R. M., 2019. Ice sheet contributions to future sea-level rise from structured expert judgment. Proc Natl Acad Sci USA 116, 11195-11200.

Barbier E. B., 2015. Climate Change Impacts on Rural Poverty in Low Elevation Coastal Zones, Policy Research Working Paper 7475 .

BCE (L. de Guindos), 2021. L'économie européenne à l'épreuve du risque systémique du changement climatique. Blog note. $11 \mathrm{p}$.

Béchet, B. (coord.), 2017. Sols artificialisés et processus d'artificialisation des sols : déterminants, impacts et leviers d'action. INRA (France), $609 \mathrm{p}$.

Beck, 2001 (1986). La société du risque. Sur la voie d'une autre modernité, trad. de l'allemand par L. Bernardi. Paris, Aubier. 521 p.

Bedsworth L., Cayan D., Franco G.; Fisher L., Ziaja S., 2018. California state wide report on $4^{\text {th }}$ climate change assessment. Governor's office of planning and research, Scripps oceanography institute et al. No Sum-CcCa4-2018-013.132 p.

Bernon N., Mallet C., Belon R., avec la collaboration de Hoareau A., Bulteau T., Garnier C., 2016. Caractérisation de l'aléa recul du trait de côte sur le littoral de la côte Nouvelle-Aquitaine aux horizons 2025 et 2050. Rapport final. BRGM/RP-66277-FR, 99 p.

Bonneil C., Fressoz J.-B., 2013. L'événement anthropocène : la Terre, l'histoire et nous. Coll. «Anthropocène ». Le Seuil. 308 p.

Bourg D, P. B. Joly et A. Kaufmann (dir.), 2013 : Du risque à la menace : penser la catastrophe. Colloque de Cerisy. PUF. Coll. « L'écologie en question ». 374 p.

Bravard J.-P., Goichot M., Gaillot S., 2013. Geography of Sand and Gravel Mining in the Lower Mekong River. In EchoGéo ; journals.openedition.org/echogeo/13659

Brecht H., Dasgupta S., Laplante B., Murray S., Wheeler D., 2012. Sea-level rise and storm surges : High stakes for a small number of developing countries. The Journal of Environment \& Development, 21 (1): 120-138.

Bren d'Amour C., Reitsma F., Baiocchi G., Barthel S., Güneralp B., Erb K. H., Haberl H., Crutzig F., Seto K. C., 2016. Future urban land expansion and implications for global croplands. Proceedings of the National Academy of Sciences, 114 (34), 8939-8944.

Buchou S., 2019. Quel littoral pour demain ?Vers un nouvel aménagement des territoires côtiers adapté au changement climatique. Rapport au Premier ministre et au MTES. Assemblée nationale. 113 p.

Burke L., Reytar K., Spalding M. and Perry A., 2011. Reefs at Risk Revisited, ISBN : 978-1-56973-762-0.

CCR (Caisse centrale de réassurance), 2018. Conséquences du changement climatique sur le coût des catastrophes naturelles en France à l'horizon 2050. Étude CCR-Météo-France RCP 8,5. 32 p. 
Cassier I., Maréchal K., Méda D. (coord.), 2017. Vers une société post-croissance : intégrer les défis écologiques, économiques et sociaux. Éd. de L’aube. Coll. « Bibliothèque des savoirs ». 216 p.

Charbonnier P., 2020. "Where is Your Freedom Now ?" How the Moderns Became Ubiquitous. In : Latour B., Weibel P. (eds), Critical Zones: The Science and Politics of Landing on Earth. ZKM Centre for Art and Media.

Chaumillon É., Garnier É., Sauzeau T. (coord.), 2014. Les littoraux à l'heure du changement climatique. Colloque 18-20 nov. 2010 ; Rochefort, Brouage, La Rochelle. Éd. Les Indes savantes. Coll. « Rivages des Xantons ». $264 \mathrm{p}$.

Chen C. C., McCarl B., Chang C. C., 2012. Climate change, sea level rise and rice: global market implications. Climatic change, 110 (3), 543-560.

Chinese academy of Sciences, 2009: Marine science and technology in China: a roadmap to 2050. Science Press Beijing \& Springer. 178 p.

Chneiweiss A., Bardaji J., 2020. Les assureurs face au défi climatique. Fondation pour l'innovation politique. $56 \mathrm{p}$.

Church J. A., White N. J., 2011. Sea-Level Rise from the Late $19^{\text {th }}$ to the Early $21^{\text {st }}$ Century. Surv Geophys 32, 585-602.

Church J. A., Gregory J. M., Lambert E., Le Cozannet G., Lowe J., 2019. Meeting User Needs for Sea Level Rise Information: A Decision Analysis Perspective. Earth's Future 7, 320-337.

Clark P. U., Cazenave A., Gregory J. M., Jevrejeva S., Levermann A., Merrifield M. A., Milne G. A. et al., 2013. 'Sea level change', in: Stocker T. F., Qin D., Plattner G.-K. et al. (eds), Climate change 2013: The physical science basis. Contribution of Working Group I to the $5^{\text {th }}$ Assessment Report of the IPCC ; Cambridge Univ. Press, Cambridge ; UK and New York, USA.

Clark P. U., Shakun J. D., Markott S. A., Mix A. C., Eby M., Kulp S., Levermann A. et al., 2016. Consequences of twenty-first-century policy for multi-millennial climate and sea-level change. Nature climate change, 6 (4), 360-368.

Conway E., Oreskes N., 2014. L'effondrement de la civilisation occidentale : un texte venu du futur. Éd. LLL les liens qui libèrent. $124 \mathrm{p}$.

Dang T. D., Cochrane T. A., Arias M. E., Tri V. P. D., 2018. Future hydrological alterations in the Mekong Delta under the impact of water resources development, land subsidence and sea level rise. Journal of Hydrology : Regional Studies.

Cury P., Miserey Y., 2008. Une Mer sans poissons. Éd. Calmann-Lévy, 286 p.

Dasgupta S., Laplante B., Murray S., Wheeler D., 2011. Exposure of developing countries to sea-level rise and storm surges. Climatic Change, 106 (4): 567-579.

Dawson R. J. (ed.), 2015. A Climate Change Report Card for Infrastructure. LWEC Report Card. Living With Environmental Change.

DeConto R. M., Pollard D., 2016. Contribution of Antarctica to past and future sea-level rise, Nature volume 531, 591-597.

Delta Commissie, 2008. Working together with water. A living land builds for its future, Wilfried ten Brinke (Ed.), $134 \mathrm{p}$.

Duvat V., Mossot G., 2012. Réseau thématique pluridisciplinaire Littoral : état de la recherche. Rapport CNRS-INEE/Liens. 65 p.

Dieng H. B., Cazenave A., Meyssignac B., Ablain M., 2017. New estimate of the current rate of sea level rise from asea level budget approach, Geophys. Res. Lett., 44, 3744-3751.

Erban L. E., Gorelick S. M., Zebker H. A., 2014. Groundwater extraction, land subsidence, and sealevel rise in the Mekong Delta, Vietnam. Environ. Res. Lett. 9, 0840106 p. 
European Commission, 2020. The EU Blue Economy Report, June 2020, 180 p. https://blueindicators. ec.europa.eu/published-reports_en

Euzen A., Gaill F., Lacroix D., Cury P. (dir.), 2019. L'Océan à Découvert. CNRS Éditions 350 p.

FAO, 2020. La situation mondiale des pêches et de l'aquaculture en 2020. Résumé. La durabilité en action. Rome. 28 p.

Foster G. L., Rohling E. J., 2013. Relationship between sea level and climate forcing by $\mathrm{CO}_{2}$ on geological timescales. PNAS 110 (4), 1209-1214.

Gattuso J. P., Magnan A., Billé R., Cheung W. W. L., Howes E. L., Joos F., Allemand D, Bopp L. et al., 2015. Contrasting futures for ocean and society from different anthropogenic $\mathrm{CO}_{2}$ emissions scenario. Science o3 Jul 2015: Vol. 349, Issue 6243.

Geisler C., Currens B., 2017. Impediments to inland resettlement under conditions of accelerated sea level rise. Land Use Policy, 66, 322-330.

Gemenne F., Blocher J., 2017. How can migration serve adaptation to climate change? Challenges to fleshing out a policy ideal. The Geographical Journal, 183 (4): 336-347.

GIP Littoral Aquitain, 2012. Stratégie régionale de gestion de la bande côtière, www.littoral-aquitain. $\mathrm{fr} /$ gestion-bande-cotiere/strategie-regionale.

Godet M., Durance P., 2008. La prospective stratégique pour les entreprises et les territoires. Dunod. Paris. $160 \mathrm{p}$.

Gueben-Venière S., 2015. De l'équipement à la gestion du littoral ou comment vivre avec les aléas météo marins aux Pays-Bas ?, Géoconfluences, ENS-Lyon/dossiers-thématiques/risques-et-sociétés.

Habermas J., (1985), 2011. Le discours philosophique de la modernité. Gallimard. Coll. « Tel ». 430 p.

Hallegatte S., Green C., Nicholls R. J., Corfee-Morlot J., 2013. Future flood losses in major coastal cities. Nature climate change, 3 (9), 802.

Hanson S., Nicholls R., Ranger N., Hallegatte S., Corfee-Morlot J., Herweijer C., Chateau J., 2011. A global ranking of port cities with high exposure to climate extremes. Climatic change, 104 (1): 89-111.

Haasnoot M., Kwadijk J., Van Alphen J., Le Bars D., Van Den Hurk B., Diermanse F. et al., 2020.Adaptation to uncertain sea-level rise; how uncertainty in Antarctic mass-loss impacts the coastal adaptation strategy of the Netherlands. Environmental Research Letters, 15 (3), 034007.

Hereher, 2010. Vulnerability of the Nile Delta to sea level rise: an assessment using remote Sensing. Geomatics, Nat. Hazards Risk, 1, 315-321.

Hinkel J., Lincke D., Vafeidis A. T., Perrette M., Nicholls R. J., Tol R. S. J., Marzeion B. et al., 2014. Coastal flood damage and adaptation costs under $21^{\text {st }}$ century sea-level rise. PNAS, 111 (9), 3292-3297.

IMBIE team, 2018. Mass balance of the Antarctic ice sheet from 1997 to 2017. Nature, $558:$ 219-222.

Kälin W., 2014. Internal Displacement/People displaced by disasters In: The Oxford Handbook of Refugee and Forced Migration Studies. UK.

de Koning R., 2009. Climate Change, Land and Security. Stockholm, Sweden : Stockholm International Peace Research Institute.

IFEN, 2006. État de l'environnement en France. Min. de l'Environnement. 495 p.

IPCC, 2014. Climate Change 2014: Impacts, Adaptation, and Vulnerability, Part B : Regional Aspects. Contribution of Working Group II to the Fifth Assessment Report of the Intergovernmental Panel on Climate Change. Cambridge University Press, 68 p.

Jevrejeva S., Frederikse T., Kopp R. E., Le Cozannet G., Jackson L. P., van de Wal R. S. W., 2019. Probabilistic Sea Level Projections at the Coast by 2100. Surv Geophys, 40, 1673-1696. 
Kara M. H, Lacroix D., Sadek S., Blancheton J. P., Rey-Valette H., Kraiem M. 2016. Vingt ans d'aquaculture en Afrique du Nord : évolutions, bilan critique et avenir. Cahiers de l'Agriculture, 25: 160117.

Kopp R. E., DeConto R. M., Bader D. A., Hay C. C., Horton R. M., Kulp S. et al. 2017. Evolving Understanding of Antarctic Ice-Sheet Physics and Ambiguity in Probabilistic Sea-Level Projections. Earth's Futur, 5, 1217-1233.

Kummu M., De Moel H., Salvucci G., Viviroli D., Ward P. J., Varis O., 2016. Over the hills and further away from coast: global geospatial patterns of human and environment over the $20^{\text {th }}-21^{\text {st }}$ centuries. Environmental Research Letters, 11 (3), 034010.

Labeyrie, 2015. Submersion : comment gérer la montée du niveau des mers. Éd. O. Jacob/Sciences. $164 \mathrm{p}$.

Lacroix D., Laurent L., de Menthière N., Schmitt B., Béthinger A., David B., Didier C., Parent du Châtelet J., 2019. Multiple visions of the future and major environmental scenarios. Technological forecasting and social change, 144, 93-102.

Latour, B., 2020. Quel État peut imposer des " gestes barrières » aux catastrophes écologiques ? Esprit, 7, 159-168.

Le Bars D., Drijfhout S., de Vries H. 2017. A high-end sea level rise probabilistic projection including rapid Antarctic ice sheet mass loss. Environ. Res. Lett., 12, 044013.

Le Cozannet G., Bulteau T., Baills A., Garcin M., 2016. Conséquences du changement climatique sur les risques côtiers en Nouvelle-Aquitaine : état des connaissances. Rapport final, BRGM/ RP-66465-FR, 74 p.

Le Mouël C., Forslund A., Marty P., Manceron S., Marajo-Petitzon E., Caillaud M. A., Schmitt B., 2017. La dépendance alimentaire de l'Afrique du Nord et du Moyen-Orient à l'horizon 2050. Éd. Quae, Versailles. Coll. «Matière à débattre et à décider ». $144 \mathrm{p}$.

Le Treut H. (dir.), 2018. Anticiper les changements climatiques en Nouvelle-Aquitaine : pour agir dans les territoires, AcclimaTerra, Éditions Région Nouvelle-Aquitaine, 488 p.

Leal Filho W. 2020. Handbook of Climate Change Adaptation. Berlin, Heidelberg : Springer Nature.

Magnan A., Duvat V., 2015. La fabrique des catastrophes «naturelles ». Natures Sciences Sociétés, 23 (2), 97-108.

Mahan A. T., 1897. The interest of America in seapower, present and future. Kennikat press NY 1970. $156 \mathrm{p}$.

McGranahan G., Balk D., Anderson B., 2007. The rising tide: assessing the risks of climate change and human settlements in low elevation coastal zones. Environment and Urbanization, 19: 17-37.

Merkens J. L., Reimann L., Hinkel J., Vafeidis A. T., 2016. Gridded population projections for the coastal zone under the Shared Socioeconomic Pathways. Global and Planetary Change, 145 : 57-66.

Minderhoud P. S. J., Middelkoop H., Erkens G., Stouthamer E., 2020. Groundwater extraction may drown mega-delta: projections of extraction-induced subsidence and elevation of the Mekong delta for the $21^{\text {st }}$ century. Environmental Research Communications, 2 (1), 011005.

Mouginot J., Rignot E., Scheuchl B., 2014. Sustained increase in ice discharge from the Amundsen Sea Embayment, West Antarctica, from 1973 to 2013. Geophys. Res. Lett. 41, 1576-1584.

Mora O., 2018. Dietary Changes, Nutrition Transition and the Future of Global Diets (chap. 8). In: Land use and food security in 2050: a narrow road, Le Mouël C., Lattre-Gasquet D., Mora O., Éd. Quae. p. 113-137.

Myers N., 1993. Environmental refugees in a globally warmed world. Jstor-Bioscience, 43 (11), 752 761. Oxford Univ Press.

Neumann B., Vafeidis A. T., Zimmermann J., Nicholls R. J., 2015. Future Coastal Population Growth and Exposure to Sea Level Rise and Coastal Flooding - A Global Assessment. Plos ONE, 10 (3): e0118571. 
Nguyen T. T. X., Woodroffe, C. D., 2016. Assessing relative vulnerability to sea-level rise in the western part of the Mekong River Delta in Vietnam. In: Sustainability Science.

Nicholls R. J., Hanson S. E., Lowe J. A., Warrick R. A., Lu X., Long A. J., 2014. Sea-level scenarios for evaluating coastal impacts: Sea-level scenarios for evaluating coastal impacts. WIREs Clim Change, 5, p. 129-150.

Noin D. , 1999. « La population des littoraux du monde ». L'information géographique, 63 (2), 65-73. OECD, 2016.: The Ocean Economy in 2030. OECD Publishing, Paris. 256 p.

OECD, 2019. Responding to rising seas: OECD approaches to tackling coastal risks. OECD Publishing, Paris. $174 \mathrm{p}$.

Oppenheimer M., Glavovic B. C., Hinkel J., van de Wal R., Magnan A. K., Abd-Elgawad A., Cai R. et al., 2019. Sea Level Rise and Implications for Low-Lying Islands, Coasts and Communities. In : IPCC Special Report on the Ocean and Cryosphere in a Changing Climate [H.-O. Pörtner et al. eds.)]

Parris A. S., Bromirski P., Burkett V., Cayan D. R., Culver M. E., Hall J., Horton et al., 2012: Global sea level rise scenarios for the United States National Climate Assessment. NOAA Tech Memo OAR CPO-1. 33 p.

Pritchard H. D., Ligtenberg S. R. M., Fricker H. A., Vaughan D. G., van den Broeke M. R., Padman L., 2012. Antarctic ice-sheet loss driven by basal melting of ice shelves. Nature, 484, 502-505.

Rocle N., 2017. L'adaptation des littoraux aux changements climatiques : une gouvernance performative par expérimentations et stratégies d'action publique. Thèse de doctorat en sociologie, Univ. de Bordeaux. 345 p.

Rocle N., Rey-Valette H., Bertrand F., Becu N., Long N., Bazart C., Vye D., Meur-Ferec C. et al., 2020. Paving the way to coastal adaptation pathways : An interdisciplinary approach based on territorial archetypes. Environmental Science \& Policy. Vol 110 : 34-45.

Sadek S., 2013. Aquaculture site selection and carrying capacity estimates for inland and coastal aquaculture in the Arab Republic of Egypt. In : Ross L.G. et al. eds. Site selection and carrying capacities for inland and coastal aquaculture. FAO/loA, Univ. of Stirling, Expert Workshop, 6-8 Dec. 2010. UK. FAO Fisheries \& Aquaculture Proc. № 21, 183-196.

Sakyo K., 1977. La submersion du Japon. Coll. «Super-fiction ». Albin Michel Paris. 256 p.

Stammer D., Wal R. S. W., Nicholls R. J., Church J. A., Le Cozannet G., Lowe J. A. et al. 2019. Framework for High-End Estimates of Sea Level Rise for Stakeholder Applications. Earth's Future, 7, 923-938.

Stern N. (coord.), 2006. Stern review on the economics of climate change. HM Treasury, London. $700 \mathrm{p}$.

Syvitski J. P. M., Kettner A. J., Overeem I., Hutton É. W. H., Hannon M. T. et al., 2009. Sinking deltas. In: Nature Geoscience, 2. 681-686.

WUP (World Urbanization Prospects), 2018. Revision of the population data. United Nations, Department of Economic and Social Affairs, Population Division.

Urbina I., 2019. La jungle des océans : crimes impunis, esclavage, ultra violence, pêche illégale. Payot/Actes Sud. $602 \mathrm{p}$.

Vafeidis A. T., Nicholls R. J., McFadden L., Tol R. S. J., Hinkel J., Spencer T., Klein R. J. T., 2008. A new global coastal database for impact and vulnerability analysis to sea-level rise. Journal of Coastal Research, 24, 917-924.

Valantin J.-M., 2017. Géopolitique d'une planète déréglée. Seuil. Coll. «Choc de l'anthropocène ». 310 p.

Wong P. P., Losada I. J., Gattuso J.-P., Hinkel J., Khattabi A., McInnes K. L., Saito Y., Sallenger A., 2014. Coastal systems and low-lying areas. In : Climate Change 2014: Impacts, Adaptation, and Vulnerability. Part A : Global and Sectoral Aspects. Contribution of Working Group II to the $5^{\text {th }}$ Assessment Report of the IPCC (Field, C.B. et al. eds.). Cambridge Univ. Press, UK. 
Coordination éditoriale : Jérémie Salinger Mise en page: EliLoCom

Achevé d'imprimer en septembre 2021 par

Numéro d'impression :

Dépôt légal : septembre 2021

Imprimé en France 
D'ici 2100 au moins, l'humanité devra faire face au phénomène inéluctable de la montée du niveau de la mer. Ce phénomène va affecter de manière croissante le littoral et les zones côtières où se concentrent de nombreuses populations et activités économiques. La vulnérabilité aux submersions de cette interface entre la terre et la mer est donc très forte, avec une exposition accrue des zones de delta, des îles et des plaines côtières de faibles altitudes. La prévention des risques constitue ainsi un enjeu majeur des politiques publiques comme des acteurs privés.

À ce titre, la recherche en sciences de l'environnement se doit d'alerter et de fournir des outils pour anticiper ces évolutions et contribuer à apporter des solutions pour réduire et gérer les risques liés à ce phénomène. À l'aide des méthodes de l'analyse prospective, et en partant des principales projections du GIEC, les auteurs explorent les enjeux et les conséquences de plusieurs scénarios de montée du niveau de la mer à l'horizon 2100. Cette approche vise à éclairer les nombreux acteurs impliqués dans ces scénarios en se concentrant sur l'appui aux politiques publiques et les priorités de recherches.

L'ouvrage s'adresse aux acteurs et décideurs, publics et privés, impliqués dans les risques de la montée du niveau de la mer en France métropolitaine et ultramarine, ainsi qu'aux divers médias et au grand public désireux de s'informer sur l'avenir des zones littorales.

Denis Lacroix est ingénieur agronome spécialisé en aquaculture. II a créé en 2006 la fonction de veille et de prospective auprès de la direction générale de l'Ifremer. II co-anime divers travaux collectifs de prospective au sein de réseaux de chercheurs français et européens.

Olivier Mora, ingénieur agronome et sociologue, est spécialiste de la prospective à la Direction de l'expertise scientifique collective, à la prospective et aux études d'INRAE (DEPE) depuis 2006. II co-anime le groupe transversal de prospective de l'Alliance nationale de recherche pour l'environnement AllEnvi.

Nicolas de Menthière, ingénieur des ponts, des eaux et des forêts, est directeur de l'appui aux politiques publiques d'INRAE. II a contribué à la mise en place du groupe transversal de prospective d'AllEnvi, qu'il a co-animé de 2013 à 2019.

Audrey Béthinger, ingénieure agronome, est en charge de la coordination de prospectives et d'études agro-environnementales à la DEPE d'INRAE.

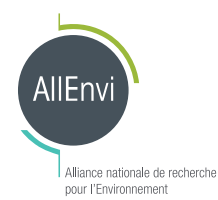

ISBN : 978-2-7592-3307-6

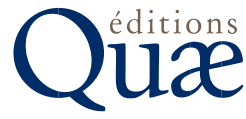

